\title{
Construction of Benzopolycycles via Pd-catalyzed Intermolecular Cyclization of 2,7-Alkadiynylic Carbonates with Terminal Propargyl Tertiary Alcohols
}

Yuchen Zhang, Chunling Fu, Xin Huang and Shengming Ma*

Laboratory of Molecular Recognition and Synthesis, Department of Chemistry, Zhejiang University, Hangzhou 310027, Zhejiang, People's Republic of China.

Fax: (+86)21-6260-9305

E-mail:masm@sioc.ac.cn

Supporting Information

\begin{tabular}{|l|l|}
\hline General Information & $\mathrm{S} 2$ \\
\hline Synthesis of starting material $\mathbf{1 h}$ & $\mathrm{S} 3-\mathrm{S} 4$ \\
\hline Synthesis of the standard products 3aa and 4aa & $\mathrm{S} 5-\mathrm{S} 6$ \\
\hline Synthesis of products 3aa $\sim$ 3pa & $\mathrm{S} 7-\mathrm{S} 31$ \\
\hline References & $\mathrm{S} 32$ \\
\hline Copies of the ${ }^{1} \mathrm{H}$ NMR, ${ }^{13} \mathrm{C}$ NMR, and HPLC spectra & $\mathrm{S} 33-\mathrm{S} 104$ \\
\hline
\end{tabular}




\section{General Information}

${ }^{1} \mathrm{H}$ NMR and ${ }^{13} \mathrm{C}$ NMR spectra were recorded in $\mathrm{CDCl}_{3}$ using a Bruker AM 300 MHz NMR spectrometer $\left({ }^{1} \mathrm{H}\right.$ at $300 \mathrm{MHz},{ }^{13} \mathrm{C}$ at $\left.75 \mathrm{MHz}\right)$. IR spectra were recorded with a Perkin-Elmer $983 \mathrm{G}$ instrument. Elemental analyses were measured with a Carlo-Erba EA1110 elementary analysis instrument. Mass spectrometry was performed with an HP 5989A system. High-resolution mass spectrometry was determined with a Finnigan MAT 8430 instrument. $\mathrm{Pd}(\mathrm{OAc})_{2}$ was purchased from Shanghai Quanzhong Chemical Technology Co., Ltd. $\mathrm{PPh}_{3}$ was purchased from Aladdin. $\mathrm{Cs}_{2} \mathrm{CO}_{3}$ was bought from Energy Chemical Reagent Co., Ltd. DMA was stirred with $\mathrm{CaH}_{2}$ and distilled right before use. Petroleum ether $\left(60-90{ }^{\circ} \mathrm{C}\right)$ was used for chromatography on silica gel. Unless otherwise indicated, chemicals and solvents were purchased from commercial suppliers. All the temperatures are referred to the oil baths used. 2,7-Alkadiynylic carbonates were prepared according to our previous literatures. ${ }^{1-3}$ The purity was determined by ${ }^{1} \mathrm{H}$ NMR spectrum using the integration of the compound and the internal standard. 


\section{Synthesis of methyl (3-methyl-1-phenyl-6-((3-phenylprop-2-yn-1-yl)oxy)hex-4-}

yn-3-yl) carbonate (1h). ${ }^{1}$ (zyc-2-23)

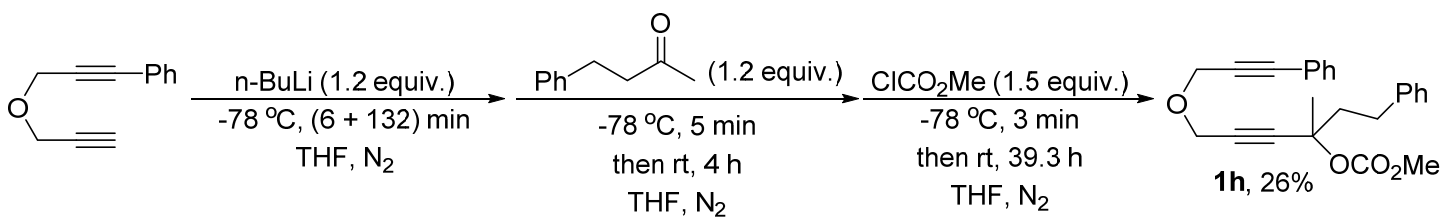

To a solution of 3-(propargyloxy)phenylpropyne $(1.7066 \mathrm{~g}, 10 \mathrm{mmol})$ in THF $(30 \mathrm{~mL})$ was added dorpwise $\mathrm{n}-\mathrm{BuLi}(2.5 \mathrm{M}$ in hexane, $4.8 \mathrm{~mL}, 12 \mathrm{mmol})$ at $-78{ }^{\circ} \mathrm{C}$ within 6 minutes. After lithiation for 132 minutes at $-78{ }^{\circ} \mathrm{C}$, 4-phenylbutan-2-one $(1.83 \mathrm{~mL}, \mathrm{~d}=0.989 \mathrm{~g} / \mathrm{mL}, 1.81 \mathrm{~g}, 12 \mathrm{mmol})$ was added dropwise with an addition funnel at $-78{ }^{\circ} \mathrm{C}$ within 5 minutes. The cooling bath was removed and the resulting mixture was warmed up to room temperature and stirred for 4 hours. Then methyl chloroformate $(1.16 \mathrm{~mL}, \mathrm{~d}=1.22 \mathrm{~g} / \mathrm{mL}, 1.42 \mathrm{~g}, 15 \mathrm{mmol})$ was added dropwise at -78 ${ }^{\circ} \mathrm{C}$ within 3 minutes. The cooling bath was removed and the resulting mixture was warmed up to room temperature and stirred for $39.3 \mathrm{~h}$. After the reaction was complete as monitored by TLC (eluent: petroleum ether/ethyl acetate/DCM = 20/1/1), it was quenched with a saturated aqueous solution of $\mathrm{NH}_{4} \mathrm{Cl}(30 \mathrm{~mL})$. After separation of the organic layer, the resulting mixture was extracted with ethyl acetate $(15 \mathrm{~mL} \times 2)$ and the combined organic phase was dried over anhydrous $\mathrm{Na}_{2} \mathrm{SO}_{4}$. After filtration and evaporation of the solvent, the crude residue was purified by chromatography on silica gel (eluent: petroleum ether/ethyl acetate/diethyl ether $=50 / 1 / 1$ ) to afford $\mathbf{1 h}$ (0.9882 g, 26\%) as a liquid: ${ }^{1} \mathrm{H}$ NMR $\left(300 \mathrm{MHz}, \mathrm{CDCl}_{3}\right) \delta$ 7.49-7.40 (m, $\left.2 \mathrm{H}, \mathrm{ArH}\right)$, 7.35-7.24 (m, $5 \mathrm{H}, \mathrm{ArH})$, 7.24-7.14 (m, $3 \mathrm{H}, \mathrm{ArH}), 4.51\left(\mathrm{~s}, 2 \mathrm{H}, \mathrm{OCH}_{2}\right), 4.41(\mathrm{~s}, 2 \mathrm{H}$, $\left.\mathrm{OCH}_{2}\right), 3.77\left(\mathrm{~s}, 3 \mathrm{H}, \mathrm{OCH}_{3}\right), 2.94-2.75\left(\mathrm{~m}, 2 \mathrm{H}, \mathrm{CH}_{2}\right), 2.36-2.22(\mathrm{~m}, 1 \mathrm{H}$, one proton 
of $\left.\mathrm{CH}_{2}\right), 2.21-2.08\left(\mathrm{~m}, 1 \mathrm{H}\right.$, one proton of $\left.\mathrm{CH}_{2}\right), 1.78\left(\mathrm{~s}, 3 \mathrm{H}, \mathrm{CH}_{3}\right) ;{ }^{13} \mathrm{C}$ NMR $(75$ $\left.\mathrm{MHz}, \mathrm{CDCl}_{3}\right) \delta 153.4,141.0,131.7,128.4,128.35,128.31,128.2,125.9,122.3,86.7$, 86.1, 84.2, 81.4, 76.8, 57.1, 56.6, 54.3, 43.1, 30.5, 26.3; IR (neat) $v\left(\mathrm{~cm}^{-1}\right) 3084,3062$, $3026,2954,2851,2238,2206,1753,1602,1490,1452,1440,1374,1351,1259,1169$, 1082, 1027; MS (EI): m/z (\%) $376\left(\mathrm{M}^{+}, 0.35\right), 317\left((\mathrm{M}-\mathrm{COOMe})^{+}, 53.54\right), 91(100)$; Anal. Calcd. for $\mathrm{C}_{24} \mathrm{H}_{24} \mathrm{O}_{4}$ (\%): C 76.57, H 6.43, Found: C 76.68, H 6.44. 
Synthesis of standard products 1,1,3,3-tetramethyl-5-phenyl-6,8-dihydrobenzo[1, 2-c:3,4-c']difuran (3aa) and 2-(7-phenyl-4-(propen-2-yl)-1,3-dihydroisobenzofuran-5-yl)propan-2-ol (4aa) under the conditions reported in our previous work. ${ }^{3}$ (zyc-1-69)

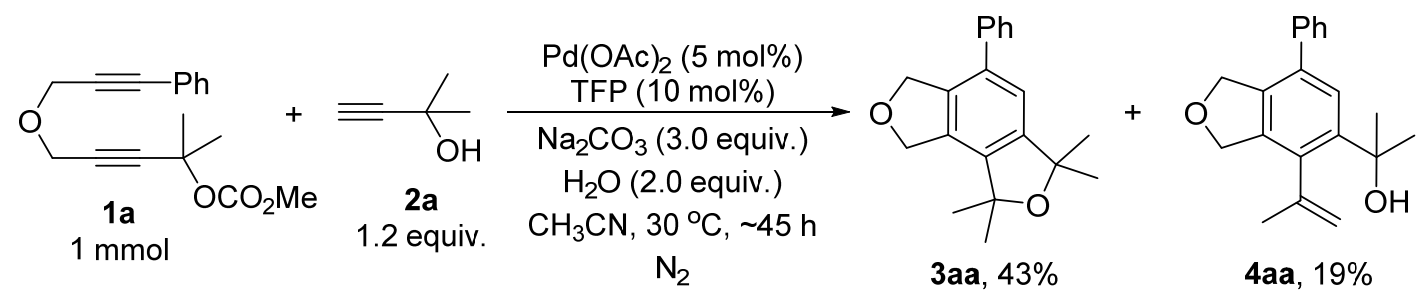

To a flame-dried Schlenk tube containing $\mathrm{Na}_{2} \mathrm{CO}_{3}(318.6 \mathrm{mg}, 3.0 \mathrm{mmol})$ were added $\mathrm{Pd}(\mathrm{OAc})_{2}(11.7 \mathrm{mg}, 0.05 \mathrm{mmol})$, TFP (23.6 mg, $\left.0.1 \mathrm{mmol}\right)$, 2a (103.7 mg, 1.2 $\mathrm{mmol}) / \mathrm{CH}_{3} \mathrm{CN}(2.0 \mathrm{~mL})$, 1a $(285.9 \mathrm{mg}, 1.0 \mathrm{mmol}) / \mathrm{CH}_{3} \mathrm{CN}(6.0 \mathrm{~mL})$, and $\mathrm{H}_{2} \mathrm{O}(36.4$ $\mathrm{mg}, 2.0 \mathrm{mmol}) / \mathrm{CH}_{3} \mathrm{CN}(2.0 \mathrm{~mL})$ sequentially under nitrogen atmosphere. The reaction was complete after being stirred at $30{ }^{\circ} \mathrm{C}$ for $\sim 45$ hours as monitored by TLC (eluent: petroleum ether/ethyl acetate $=20 / 1$ ). The resulting mixture was filtrated through a short column $(3 \mathrm{~cm})$ of silica gel eluted with ethyl acetate $(20 \mathrm{~mL} \times 3)$. After evaporation, the crude residue was purified by chromatography on silica gel (eluent: petroleum ether/ethyl acetate $=20 / 1)$ to afford 3aa $(125.4 \mathrm{mg}, 43 \%)$ and 4aa (55.7 mg, 19\%).

3aa: liquid; ${ }^{1} \mathrm{H}$ NMR $\left(300 \mathrm{MHz}, \mathrm{CDCl}_{3}\right) \delta$ 7.49-7.32 (m, $\left.5 \mathrm{H}, \mathrm{ArH}\right), 7.04$ (s, $1 \mathrm{H}$, ArH), 5.25-5.12 (m, $\left.4 \mathrm{H}, \mathrm{OCH}_{2} \times 2\right), 1.57\left(\mathrm{~s}, 6 \mathrm{H}, \mathrm{CH}_{3} \times 2\right), 1.54\left(\mathrm{~s}, 6 \mathrm{H}, \mathrm{CH}_{3} \times 2\right)$; ${ }^{13} \mathrm{C} \mathrm{NMR}\left(75 \mathrm{MHz}, \mathrm{CDCl}_{3}\right) \delta 147.1,140.1,138.3,137.3,135.7,132.4,128.6,127.8$, 127.5, 120.4, 84.6, 83.7, 73.1, 71.2, 30.9, 28.9; IR (neat) $v\left(\mathrm{~cm}^{-1}\right) 3049,3029,2971$, $2925,2855,1601,1574,1500,1475,1449,1373,1361,1342,1283,1246,1176,1138$, 
1104, 1057; MS (EI): m/z (\%) $294\left(\mathrm{M}^{+}, 0.95\right), 279\left(\left(\mathrm{M}-\mathrm{CH}_{3}\right)^{+}, 100\right)$; HRMS calcd. for $\mathrm{C}_{20} \mathrm{H}_{22} \mathrm{O}_{2}\left[\mathrm{M}^{+}\right]:$294.1620; Found: 294.1617.

4aa: liquid; ${ }^{1} \mathrm{H}$ NMR $\left(300 \mathrm{MHz}, \mathrm{CDCl}_{3}\right) \delta$ 7.48-7.31 (m, $\left.6 \mathrm{H}, \mathrm{ArH}\right)$, 5.30-5.24 (m, $1 \mathrm{H}$, one proton of $\left.=\mathrm{CH}_{2}\right), 5.23-5.18\left(\mathrm{~m}, 2 \mathrm{H}, \mathrm{OCH}_{2}\right), 5.09-5.03\left(\mathrm{~m}, 2 \mathrm{H}, \mathrm{OCH}_{2}\right)$, 4.97-4.92 (m, $1 \mathrm{H}$, one proton of $\left.=\mathrm{CH}_{2}\right), 2.85($ br s, $1 \mathrm{H}, \mathrm{OH}), 2.16\left(\mathrm{~s}, 3 \mathrm{H}, \mathrm{CH}_{3}\right), 1.67$ (s, $\left.3 \mathrm{H}, \mathrm{CH}_{3}\right), 1.66\left(\mathrm{~s}, 3 \mathrm{H}, \mathrm{CH}_{3}\right) ;{ }^{13} \mathrm{C} \mathrm{NMR}\left(75 \mathrm{MHz}, \mathrm{CDCl}_{3}\right) \delta 147.4,145.8,140.0$, $139.0,135.1,134.2,133.9,128.7,127.7,127.4,126.2,115.1,74.8,73.9,73.3,34.1$, 31.8, 24.9; IR (neat) $v\left(\mathrm{~cm}^{-1}\right) 3446,2972,2912,2854,1470,1445,1361,1263,1233$, 1148, 1058; MS (EI): m/z (\%) $294\left(\mathrm{M}^{+}, 100\right), 279\left(\left(\mathrm{M}-\mathrm{CH}_{3}\right)^{+}, 76.63\right)$; HRMS calcd. for $\mathrm{C}_{20} \mathrm{H}_{22} \mathrm{O}_{2}\left[\mathrm{M}^{+}\right]$: 294.1620; Found: 294.1621. 


\section{Synthesis of products 3 .}

1. Synthesis of 1,1,3,3-tetramethyl-5-phenyl-1,3,6,8-tetrahydrobenzo[1,2-c:3,4-c']difuran (3aa). ( zyc-1-173)
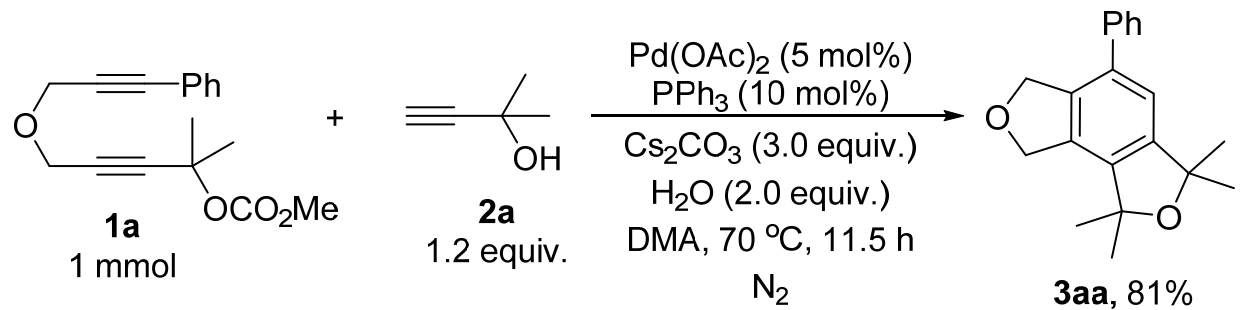

3aa, $81 \%$

Typical Procedure: To a flame-dried Schlenk tube containing $\mathrm{Cs}_{2} \mathrm{CO}_{3}(975.2$ $\mathrm{mg}, 3.0 \mathrm{mmol})$ were added $\mathrm{Pd}(\mathrm{OAc})_{2}(11.2 \mathrm{mg}, 0.05 \mathrm{mmol}), \mathrm{PPh}_{3}(27.0 \mathrm{mg}, 0.1$ mmol), 2a (102.8 mg, 1.2 mmol)/DMA (2.0 mL), 1a (286.2 mg, 1.0 mmol)/DMA (6.0 $\mathrm{mL})$, and $\mathrm{H}_{2} \mathrm{O}(36.2 \mathrm{mg}, 2.0 \mathrm{mmol}) / \mathrm{DMA}(2.0 \mathrm{~mL})$ sequentially under nitrogen atmosphere. The reaction was complete after being stirred at $70{ }^{\circ} \mathrm{C}$ for 11.5 hours as monitored by TLC (eluent: petroleum ether/ethyl acetate/DCM $=20 / 1 / 1$ ). The resulting mixture was diluted with ethyl acetate $(30 \mathrm{~mL})$ and then transferred to a separation funnel. A diluted solution of $\mathrm{HCl}(3 \mathrm{M}, 30 \mathrm{~mL})$ was added. After separation of the organic phase, the aqueous phase was extracted with ethyl acetate $(20 \mathrm{~mL} \times 2)$. The combined organic phase was washed with water $(30 \mathrm{~mL})$ and brine $(30 \mathrm{~mL})$ sequentially and dried over anhydrous $\mathrm{Na}_{2} \mathrm{SO}_{4}$. After filtration and evaporation of the solvent, the crude residue was purified by chromatography on silica gel (eluent: petroleum ether/ethyl acetate $=20 / 1)$ to afford 3aa $(238.7 \mathrm{mg}, 81 \%)$ as a liquid: ${ }^{1} \mathrm{H}$ NMR (300 MHz, $\left.\mathrm{CDCl}_{3}\right) \delta$ 7.50-7.32 (m, $\left.5 \mathrm{H}, \mathrm{ArH}\right), 7.04(\mathrm{~s}, 1 \mathrm{H}, \mathrm{ArH})$, 5.22-5.14 (m, $\left.4 \mathrm{H}, \mathrm{OCH}_{2} \times 2\right), 1.57\left(\mathrm{~s}, 6 \mathrm{H}, \mathrm{CH}_{3} \times 2\right), 1.55\left(\mathrm{~s}, 6 \mathrm{H}, \mathrm{CH}_{3} \times 2\right) ;{ }^{13} \mathrm{C} \mathrm{NMR}(75 \mathrm{MHz}$, $\left.\mathrm{CDCl}_{3}\right) \delta 147.0,140.1,138.3,137.3,135.7,132.4,128.6,127.8,127.5,120.4,84.6$, 
$83.7,73.1,71.2,30.9,28.9$.

The following compounds 3ab $\sim$ 3oa were prepared according to this Typical

\section{Procedure.}

2. Synthesis of 3-isobutyl-1,1,3-trimethyl-5-phenyl-1,3,6,8-tetrahydrobenzo[1,2-c:3,4 $\left.-c^{\prime}\right]$ difuran (3ab). (zyc-2-17)
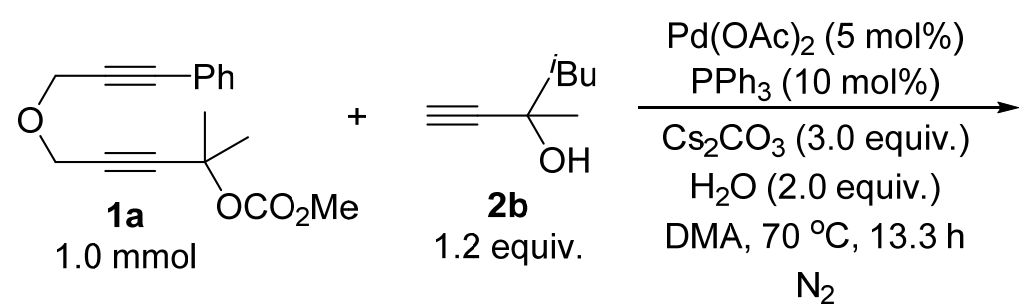

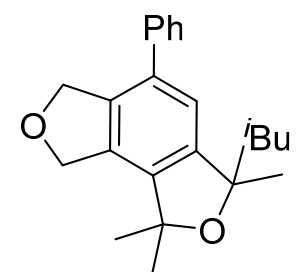

$3 a b, 67 \%$

Following Typical Procedure, the reaction of $\mathrm{Cs}_{2} \mathrm{CO}_{3}(975.9 \mathrm{mg}, 3.0 \mathrm{mmol}$ ), $\mathrm{Pd}(\mathrm{OAc})_{2}(11.2 \mathrm{mg}, 0.05 \mathrm{mmol}), \mathrm{PPh}_{3}(26.9 \mathrm{mg}, 0.1 \mathrm{mmol}), 2 \mathbf{b}(151.5 \mathrm{mg}, 1.2 \mathrm{mmol})$, 1a $(285.8 \mathrm{mg}, 1.0 \mathrm{mmol})$, and $\mathrm{H}_{2} \mathrm{O}(36.3 \mathrm{mg}, 2.0 \mathrm{mmol})$ in DMA $(10 \mathrm{~mL})$ afforded 3ab (226.6 mg, 67\%) (eluent: petroleum ether/ethyl acetate/ethyl ether $=100 / 2 / 1)$ as a liquid: ${ }^{1} \mathrm{H}$ NMR $\left(300 \mathrm{MHz}, \mathrm{CDCl}_{3}\right) \delta$ 7.48-7.27 (m, $\left.5 \mathrm{H}, \mathrm{ArH}\right), 7.00$ (s, $\left.1 \mathrm{H}, \mathrm{ArH}\right)$, 5.27-5.10 (m, $\left.4 \mathrm{H}, \mathrm{OCH}_{2} \times 2\right), 1.88-1.62\left(\mathrm{~m}, 3 \mathrm{H}, \mathrm{CH}_{2}\right.$ and $\left.\mathrm{CH}\right), 1.55\left(\mathrm{~s}, 3 \mathrm{H}, \mathrm{CH}_{3}\right)$, $1.54\left(\mathrm{~s}, 3 \mathrm{H}, \mathrm{CH}_{3}\right), 1.52\left(\mathrm{~s}, 3 \mathrm{H}, \mathrm{CH}_{3}\right), 0.97\left(\mathrm{~d}, J=6.3 \mathrm{~Hz}, 3 \mathrm{H}, \mathrm{CH}_{3}\right), 0.79(\mathrm{~d}, J=6.3$ $\left.\mathrm{Hz}, 3 \mathrm{H}, \mathrm{CH}_{3}\right) ;{ }^{13} \mathrm{C} \mathrm{NMR}\left(75 \mathrm{MHz}, \mathrm{CDCl}_{3}\right) \delta 146.5,140.1,138.6,137.2,135.4,132.2$, $128.5,127.7,127.3,120.6,87.4,83.6,73.0,71.1,50.9,30.5,29.2,28.0,25.0,24.4$, 24.2; IR (neat) $v\left(\mathrm{~cm}^{-1}\right) 3058,3029,2970,2946,2925,2866,1601,1575,1473,1446$, 1403, 1369, 1359, 1282, 1225, 1164, 1139, 1058; MS (EI): m/z (\%) $336\left(\mathrm{M}^{+}, 2.15\right)$, 279 (100); HRMS calcd. for $\mathrm{C}_{23} \mathrm{H}_{28} \mathrm{O}_{2}\left[\mathrm{M}^{+}\right]$: 336.2089; Found: 336.2092. 
3. Synthesis of 1,1,3-trimethyl-3-pentyl-5-phenyl-1,3,6,8-tetrahydrobenzo[1,2-c:3,4

$\left.-c^{\prime}\right]$ difuran (3ac). (zyc-2-32)

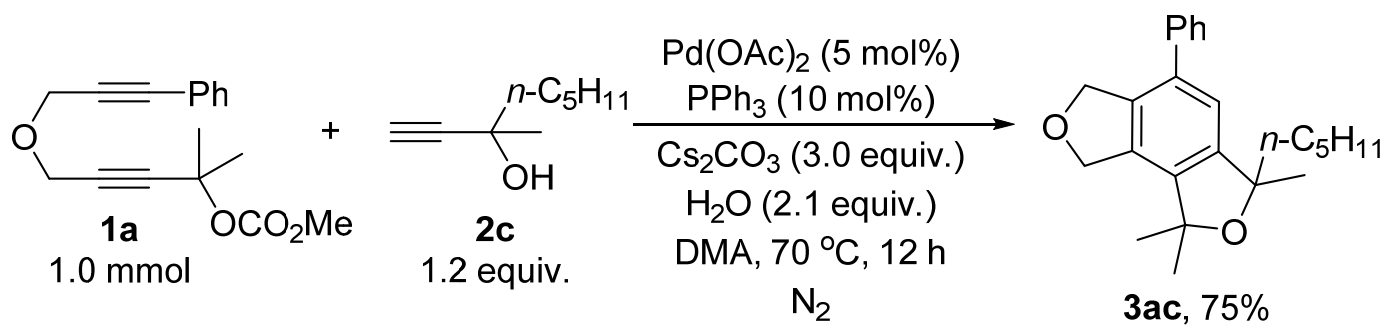

Following Typical Procedure, the reaction of $\mathrm{Cs}_{2} \mathrm{CO}_{3}(976.3 \mathrm{mg}, 3.0 \mathrm{mmol})$, $\mathrm{Pd}(\mathrm{OAc})_{2}(11.3 \mathrm{mg}, 0.05 \mathrm{mmol}), \mathrm{PPh}_{3}(26.5 \mathrm{mg}, 0.1 \mathrm{mmol}), 2 \mathrm{c}(169.5 \mathrm{mg}, 1.2 \mathrm{mmol})$, 1a $(284.1 \mathrm{mg}, 1.0 \mathrm{mmol})$, and $\mathrm{H}_{2} \mathrm{O}(37.1 \mathrm{mg}, 2.1 \mathrm{mmol})$ in DMA (10 mL) afforded 3ac $(261.0 \mathrm{mg}, 75 \%)$ (eluent: petroleum ether/ethyl acetate $=50 / 1)$ as a liquid: ${ }^{1} \mathrm{H}$ NMR (300 MHz, $\left.\mathrm{CDCl}_{3}\right) \delta$ 7.48-7.32 (m, $\left.5 \mathrm{H}, \mathrm{ArH}\right), 6.99(\mathrm{~s}, 1 \mathrm{H}, \mathrm{ArH}), 5.25-5.10(\mathrm{~m}$, $\left.4 \mathrm{H}, \mathrm{OCH}_{2} \times 2\right), 1.90-1.69\left(\mathrm{~m}, 2 \mathrm{H}, \mathrm{CH}_{2}\right), 1.54\left(\mathrm{~s}, 6 \mathrm{H}, \mathrm{CH}_{3} \times 2\right), 1.53\left(\mathrm{~s}, 3 \mathrm{H}, \mathrm{CH}_{3}\right)$, 1.50-1.36 (m, $1 \mathrm{H}$, one proton of $\left.\mathrm{CH}_{2}\right), 1.35-1.20\left(\mathrm{~m}, 4 \mathrm{H}, \mathrm{CH}_{2} \times 2\right), 1.20-1.04(\mathrm{~m}, 1$ $\mathrm{H}$, one proton of $\left.\mathrm{CH}_{2}\right), 0.85\left(\mathrm{t}, J=6.9 \mathrm{~Hz}, 3 \mathrm{H}, \mathrm{CH}_{3}\right) ;{ }^{13} \mathrm{C} \mathrm{NMR}\left(75 \mathrm{MHz}, \mathrm{CDCl}_{3}\right) \delta$ $146.0,140.2,138.8,137.2,135.6,132.3,128.6,127.8,127.4,120.5,87.2,83.5,73.1$, 71.2, 42.4, 32.1, 30.0, 29.2, 28.1, 24.1, 22.5, 14.0; IR (neat) $v\left(\mathrm{~cm}^{-1}\right) 3060,3029,2968$, 2930, 2859, 1601, 1576, 1501, 1473, 1457, 1403, 1374, 1359, 1344, 1282, 1163, 1139, 1114, 1058; MS (EI): m/z (\%) $350\left(\mathrm{M}^{+}, 2.15\right), 280$ (100); HRMS calcd. for $\mathrm{C}_{24} \mathrm{H}_{30} \mathrm{O}_{2}$ $\left[\mathrm{M}^{+}\right]$: 350.2246; Found: 350.2250.

4. Synthesis of 1,1,3-trimethyl-3-nonyl-5-phenyl-1,3,6,8-tetrahydrobenzo[1,2-c:3,4-c'] difuran (3ad). (zyc-2-33) 


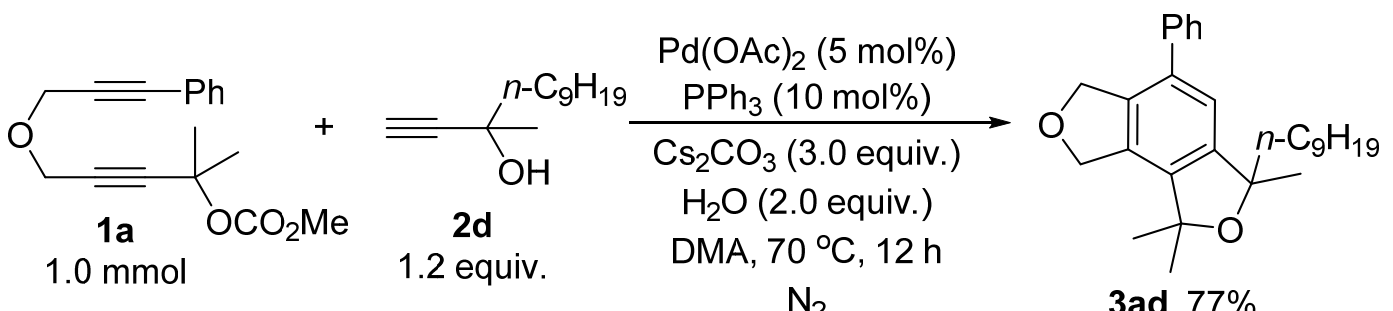

Following Typical Procedure, the reaction of $\mathrm{Cs}_{2} \mathrm{CO}_{3}(976.9 \mathrm{mg}, 3.0 \mathrm{mmol})$, $\mathrm{Pd}(\mathrm{OAc})_{2}(11.5 \mathrm{mg}, 0.05 \mathrm{mmol}), \mathrm{PPh}_{3}(26.9 \mathrm{mg}, 0.1 \mathrm{mmol}), 2 \mathrm{~d}(235.5 \mathrm{mg}, 1.2 \mathrm{mmol})$, 1a (286.0 mg, $1.0 \mathrm{mmol})$, and $\mathrm{H}_{2} \mathrm{O}(36.0 \mathrm{mg}, 2.0 \mathrm{mmol})$ in DMA (10 mL) afforded 3ad $(313.1 \mathrm{mg}, 77 \%)$ (eluent: petroleum ether/ethyl acetate $=50 / 1)$ as a liquid: ${ }^{1} \mathrm{H}$ NMR (300 MHz, $\left.\mathrm{CDCl}_{3}\right) \delta$ 7.48-7.28 (m, $\left.5 \mathrm{H}, \mathrm{ArH}\right), 6.99(\mathrm{~s}, 1 \mathrm{H}, \mathrm{ArH})$, 5.25-5.10 (m, $\left.4 \mathrm{H}, \mathrm{OCH}_{2} \times 2\right), 1.90-1.69\left(\mathrm{~m}, 2 \mathrm{H}, \mathrm{CH}_{2}\right), 1.54\left(\mathrm{~s}, 6 \mathrm{H}, \mathrm{CH}_{3} \times 2\right), 1.53\left(\mathrm{~s}, 3 \mathrm{H}, \mathrm{CH}_{3}\right)$, 1.50-1.35 (m, $1 \mathrm{H}$, one proton of $\left.\mathrm{CH}_{2}\right), 1.34-1.18\left(\mathrm{~m}, 12 \mathrm{H}, \mathrm{CH}_{2} \times 6\right), 1.18-1.02(\mathrm{~m}, 1$ $\mathrm{H}$, one proton of $\left.\mathrm{CH}_{2}\right), 0.86\left(\mathrm{t}, J=6.6 \mathrm{~Hz}, 3 \mathrm{H}, \mathrm{CH}_{3}\right) ;{ }^{13} \mathrm{C} \mathrm{NMR}\left(75 \mathrm{MHz}, \mathrm{CDCl}_{3}\right) \delta$ $146.0,140.2,138.8,137.2,135.6,132.3,128.6,127.8,127.4,120.5,87.2,83.5,73.1$, $71.2,42.5,31.8,30.0,29.9,29.5,29.4,29.2,28.1,24.4,22.6,14.0$; IR (neat) $v\left(\mathrm{~cm}^{-1}\right)$ 3060, 3029, 2968, 2925, 2853, 1601, 1576, 1501, 1466, 1457, 1403, 1374, 1359, 1344, 1282, 1177, 1159, 1139, 1058, 1030; MS (EI): m/z (\%) $406\left(\mathrm{M}^{+}, 1.45\right), 280(100)$; HRMS calcd. for $\mathrm{C}_{28} \mathrm{H}_{38} \mathrm{O}_{2}\left[\mathrm{M}^{+}\right]$: 406.2872; Found: 406.2874 .

5. Synthesis of 1,1-dimethyl-5-phenyl-6,8-dihydro- $1 H$-spiro[benzo[1,2-c:3,4-c']difuran-3,1'-cyclobutane] (3ae). (zyc-1-176) 

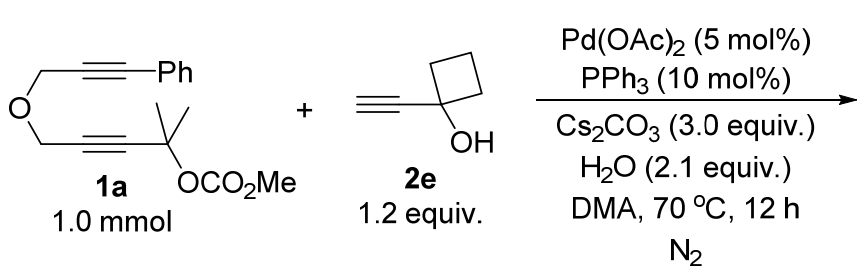

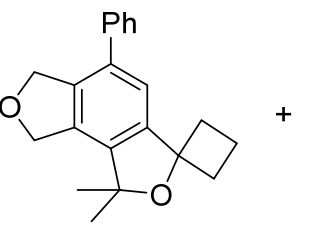

3ae, $70 \%$

Following Typical Procedure, the reaction of $\mathrm{Cs}_{2} \mathrm{CO}_{3}(975.4 \mathrm{mg}, 3.0 \mathrm{mmol})$,

$\mathrm{Pd}(\mathrm{OAc})_{2}(11.2 \mathrm{mg}, 0.05 \mathrm{mmol}), \mathrm{PPh}_{3}(26.9 \mathrm{mg}, 0.1 \mathrm{mmol}), 2 \mathrm{e}(115.2 \mathrm{mg}, 1.2 \mathrm{mmol})$,

1a $(285.6 \mathrm{mg}, 1.0 \mathrm{mmol})$, and $\mathrm{H}_{2} \mathrm{O}(37.1 \mathrm{mg}, 2.1 \mathrm{mmol})$ in DMA $(10 \mathrm{~mL})$ afforded 3ae $(213.5 \mathrm{mg}, 70 \%)$ and 4ae $(32.0 \mathrm{mg}$, purity $=98 \%, 10 \%)$ (eluent: petroleum ether/ethyl acetate $=20 / 1(630 \mathrm{~mL})$ to $10 / 1(440 \mathrm{~mL})$ to $5 / 1(240 \mathrm{~mL}))$.

3ae: solid; m.p. $127.1-127.7{ }^{\circ} \mathrm{C}$ (n-hexane/DCM); ${ }^{1} \mathrm{H}$ NMR $\left(300 \mathrm{MHz}, \mathrm{CDCl}_{3}\right) \delta$ 7.51-7.32 (m, $6 \mathrm{H}, \mathrm{ArH}), 5.17\left(\mathrm{~s}, 4 \mathrm{H}, \mathrm{OCH}_{2} \times 2\right), 2.73-2.57\left(\mathrm{~m}, 2 \mathrm{H}, \mathrm{CH}_{2}\right), 2.52-2.38$ $\left(\mathrm{m}, 2 \mathrm{H}, \mathrm{CH}_{2}\right), 2.06-1.85\left(\mathrm{~m}, 2 \mathrm{H}, \mathrm{CH}_{2}\right), 1.49\left(\mathrm{~s}, 6 \mathrm{H}, \mathrm{CH}_{3} \times 2\right) ;{ }^{13} \mathrm{C} \mathrm{NMR}(75 \mathrm{MHz}$, $\left.\mathrm{CDCl}_{3}\right) \delta 146.2,140.2,138.8,137.3,136.0,131.8,128.7,127.9,127.5,119.5,86.1$, 84.1, 73.1, 71.2, 38.9, 28.0, 13.2; IR (KBr) $v\left(\mathrm{~cm}^{-1}\right)$ 3053, 3028, 2983, 2965, 2929, 2860, 2826, 1600, 1575, 1473, 1454, 1446, 1424, 1406, 1376, 1362, 1348, 1282, 1261 , 1242, 1188, 1166, 1127, 1105, 1074, 1051, 1029; MS (EI): m/z (\%) $306\left(\mathrm{M}^{+}, 3.24\right)$, 263 (100.0); Anal. Calcd. for $\mathrm{C}_{21} \mathrm{H}_{22} \mathrm{O}_{2}$ (\%): C 82.32, H 7.24, Found: C 82.16, H 7.25. 4ae: liquid; ${ }^{1} \mathrm{H}$ NMR $\left(300 \mathrm{MHz}, \mathrm{CDCl}_{3}\right) \delta$ 7.50-7.32 (m, $\left.5 \mathrm{H}, \mathrm{ArH}\right), 7.27$ (s, $1 \mathrm{H}$, ArH), 5.34-5.26 (m,1 H, one proton of $\left.=\mathrm{CH}_{2}\right), 5.25-5.14\left(\mathrm{~m}, 2 \mathrm{H}, \mathrm{OCH}_{2}\right), 5.13-5.02$ $\left(\mathrm{m}, 2 \mathrm{H}, \mathrm{OCH}_{2}\right), 5.02-4.92\left(\mathrm{~m}, 1 \mathrm{H}\right.$, one proton of $\left.=\mathrm{CH}_{2}\right), 2.77-2.55\left(\mathrm{~m}, 2 \mathrm{H}, \mathrm{CH}_{2}\right)$, 2.47 (br s, $1 \mathrm{H}, \mathrm{OH}), 2.41-2.19\left(\mathrm{~m}, 3 \mathrm{H}, \mathrm{CH}_{2}\right.$ and one proton of $\left.\mathrm{CH}_{2}\right), 2.11(\mathrm{~s}, 3 \mathrm{H}$, $\left.\mathrm{CH}_{3}\right), 1.87-1.70\left(\mathrm{~m}, 1 \mathrm{H}\right.$, one proton of $\left.\mathrm{CH}_{2}\right) ;{ }^{13} \mathrm{C} \mathrm{NMR}\left(75 \mathrm{MHz}, \mathrm{CDCl}_{3}\right) \delta$ 144.6, $143.2,139.9,139.0,136.1,135.1,134.3,128.7,127.8,127.5,125.6,116.5,79.1,73.7$, 
73.1, 36.7, 24.0, 15.6; IR (neat) $v\left(\mathrm{~cm}^{-1}\right) 3422,2948,2854,1638,1600,1472,1446$, 1361, 1278, 1253, 1219, 1146, 1130, 1058; MS (EI): m/z (\%) $306\left(\mathrm{M}^{+}, 13.90\right), 278$ (100); HRMS calcd. for $\mathrm{C}_{21} \mathrm{H}_{22} \mathrm{O}_{2}\left[\mathrm{M}^{+}\right]$: 306.1620, Found: 306.1624 .

The gram-scale reaction of 3ae. (zyc-1-194)
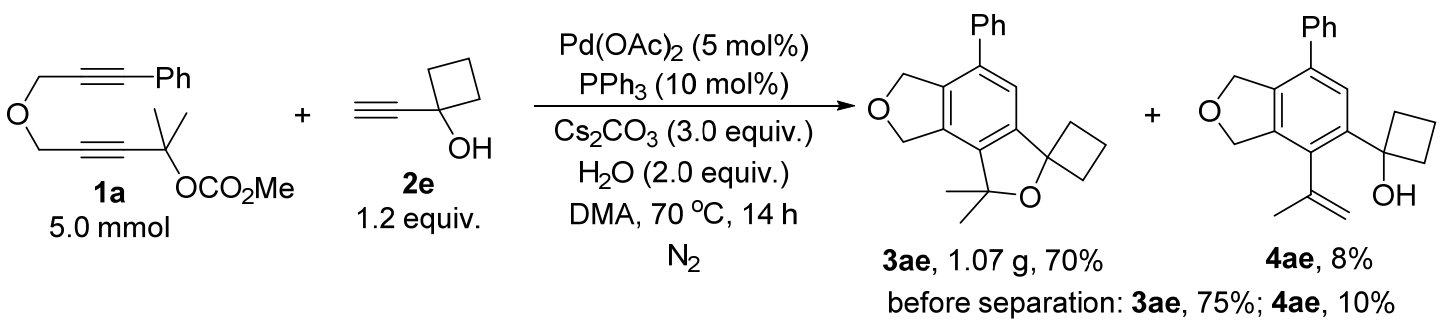

Following Typical Procedure, the reaction of $\mathrm{Cs}_{2} \mathrm{CO}_{3}(4877.0 \mathrm{mg}, 15.0 \mathrm{mmol})$, $\mathrm{Pd}(\mathrm{OAc})_{2}(56.0 \mathrm{mg}, 0.25 \mathrm{mmol}), \mathrm{PPh}_{3}(131.2 \mathrm{mg}, 0.5 \mathrm{mmol}), 2 \mathrm{e}(576.3 \mathrm{mg}, 6.0$ mmol), 1a (1429.8 mg, $5.0 \mathrm{mmol})$, and $\mathrm{H}_{2} \mathrm{O}(180.1 \mathrm{mg}, 10.0 \mathrm{mmol})$ in DMA (50 mL) afforded 3ae (1074.3 mg, 70\%) and 4ae (119.2 mg, purity = 98\%, 8\%) (eluent: petroleum ether/ethyl acetate $=40 / 1(1300 \mathrm{~mL})$ to $15 / 1(1500 \mathrm{~mL}))$.

3ae: solid; ${ }^{1} \mathrm{H}$ NMR $\left(300 \mathrm{MHz}, \mathrm{CDCl}_{3}\right) \delta$ 7.51-7.32 (m, $\left.6 \mathrm{H}, \mathrm{ArH}\right), 5.17(\mathrm{~s}, 4 \mathrm{H}$, $\left.\mathrm{OCH}_{2} \times 2\right), 2.75-2.57\left(\mathrm{~m}, 2 \mathrm{H}, \mathrm{CH}_{2}\right), 2.52-2.38\left(\mathrm{~m}, 2 \mathrm{H}, \mathrm{CH}_{2}\right), 2.06-1.85(\mathrm{~m}, 2 \mathrm{H}$, $\left.\mathrm{CH}_{2}\right), 1.49\left(\mathrm{~s}, 6 \mathrm{H}, \mathrm{CH}_{3} \times 2\right) ;{ }^{13} \mathrm{C} \mathrm{NMR}\left(75 \mathrm{MHz}, \mathrm{CDCl}_{3}\right) \delta 146.2,140.2,138.8,137.3$, $136.0,131.8,128.7,127.9,127.5,119.4,86.1,84.1,73.1,71.2,38.8,28.0,13.2$.

4ae: liquid; ${ }^{1} \mathrm{H}$ NMR $\left(300 \mathrm{MHz}, \mathrm{CDCl}_{3}\right) \delta$ 7.50-7.29 (m, $\left.5 \mathrm{H}, \mathrm{ArH}\right), 7.26$ (s, $1 \mathrm{H}$, ArH), 5.34-5.23 (m, $1 \mathrm{H}$, one proton of $\left.=\mathrm{CH}_{2}\right), 5.22-5.12\left(\mathrm{~m}, 2 \mathrm{H}, \mathrm{OCH}_{2}\right), 5.10-4.98$ $\left(\mathrm{m}, 2 \mathrm{H}, \mathrm{OCH}_{2}\right), 4.98-4.90\left(\mathrm{~m}, 1 \mathrm{H}\right.$, one proton of $\left.=\mathrm{CH}_{2}\right), 2.82-2.45\left(\mathrm{~m}, 3 \mathrm{H}, \mathrm{CH}_{2}\right.$ and $\mathrm{OH}), 2.42-2.15\left(\mathrm{~m}, 3 \mathrm{H}, \mathrm{CH}_{2}\right.$ and one proton of $\left.\mathrm{CH}_{2}\right), 2.09\left(\mathrm{~s}, 3 \mathrm{H}, \mathrm{CH}_{3}\right), 1.85-1.68$ (m, $1 \mathrm{H}$, one proton of $\left.\mathrm{CH}_{2}\right) ;{ }^{13} \mathrm{C} \mathrm{NMR}\left(75 \mathrm{MHz}, \mathrm{CDCl}_{3}\right) \delta 144.4,143.2,139.8,138.9$, $135.9,135.1,134.2,128.6,127.7,127.4,125.6,116.4,79.0,73.6,73.0,36.5,24.0$, 
6. Synthesis of 1,1-dimethyl-5-phenyl-6,8-dihydro-1H-spiro[benzo[1,2-c:3,4-c']difuran-3,1'-cyclooctane] (3af). (zyc-1-186)
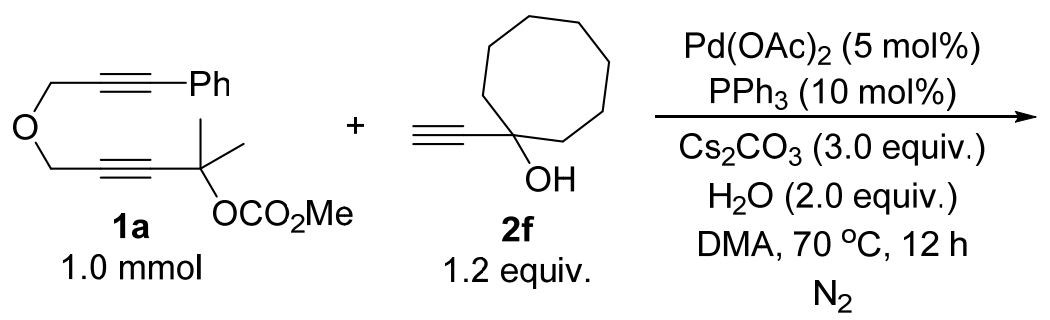

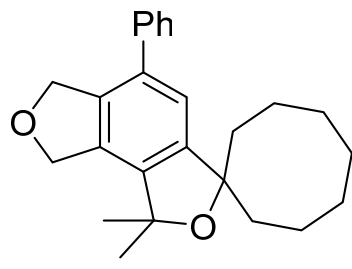

3af, $71 \%$

Following Typical Procedure, the reaction of $\mathrm{Cs}_{2} \mathrm{CO}_{3}(976.4 \mathrm{mg}, 3.0 \mathrm{mmol})$,

$\mathrm{Pd}(\mathrm{OAc})_{2}(11.8 \mathrm{mg}, 0.05 \mathrm{mmol}), \mathrm{PPh}_{3}(26.6 \mathrm{mg}, 0.1 \mathrm{mmol}), 2 \mathrm{f}(182.7 \mathrm{mg}, 1.2 \mathrm{mmol})$,

1a $(286.2 \mathrm{mg}, 1.0 \mathrm{mmol})$, and $\mathrm{H}_{2} \mathrm{O}(36.8 \mathrm{mg}, 2.0 \mathrm{mmol})$ in DMA $(10 \mathrm{~mL})$ afforded 3af $(255.6 \mathrm{mg}, 71 \%)$ (eluent: petroleum ether/ethyl acetate $=20 / 1)$ as a solid: m.p. 132.5-134.0 ${ }^{\circ} \mathrm{C}$ (n-hexane/DCM); ${ }^{1} \mathrm{H}$ NMR $\left(300 \mathrm{MHz}, \mathrm{CDCl}_{3}\right) \delta$ 7.52-7.30 (m, $5 \mathrm{H}$, ArH), 7.09 (s, $1 \mathrm{H}, \mathrm{ArH})$, 5.25-5.10 (m, $\left.4 \mathrm{H}, \mathrm{OCH}_{2} \times 2\right), 2.16-2.01\left(\mathrm{~m}, 2 \mathrm{H}, \mathrm{CH}_{2}\right)$, 1.98-1.86 (m, $\left.2 \mathrm{H}, \mathrm{CH}_{2}\right), 1.86-1.64\left(\mathrm{~m}, 7 \mathrm{H}, \mathrm{CH}_{2} \times 3\right.$ and one proton of $\left.\mathrm{CH}_{2}\right)$, 1.64-1.45 (m, $3 \mathrm{H}, \mathrm{CH}_{2}$, one proton of $\left.\mathrm{CH}_{2}\right), 1.52\left(\mathrm{~s}, 6 \mathrm{H}, \mathrm{CH}_{3} \times 2\right) ;{ }^{13} \mathrm{C}$ NMR $(75$ $\left.\mathrm{MHz}, \mathrm{CDCl}_{3}\right) \delta 147.6,140.3,138.7,137.2,135.2,132.4,128.6,127.9,127.4,120.8$, 89.2, 83.2, 73.1, 71.2, 38.6, 29.1, 28.3, 25.0, 22.3; IR (KBr) v $\left(\mathrm{cm}^{-1}\right)$ 3032, 2966, 2925, 2854, 1600, 1470, 1443, 1403, 1357, 1334, 1281, 1160, 1135, 1079, 1055, 1032, 1004; MS (EI): m/z (\%) $362\left(\mathrm{M}^{+}, 32.27\right), 291$ (100); Anal. Calcd. for $\mathrm{C}_{25} \mathrm{H}_{30} \mathrm{O}_{2}(\%): \mathrm{C} 82.83$, H 8.34, Found: C 82.76, H 8.25.

7. Synthesis of 5-(4-methoxyphenyl)-1,1-dimethyl-6,8-dihydro- $1 H$-spiro[benzo[1,2-c: 

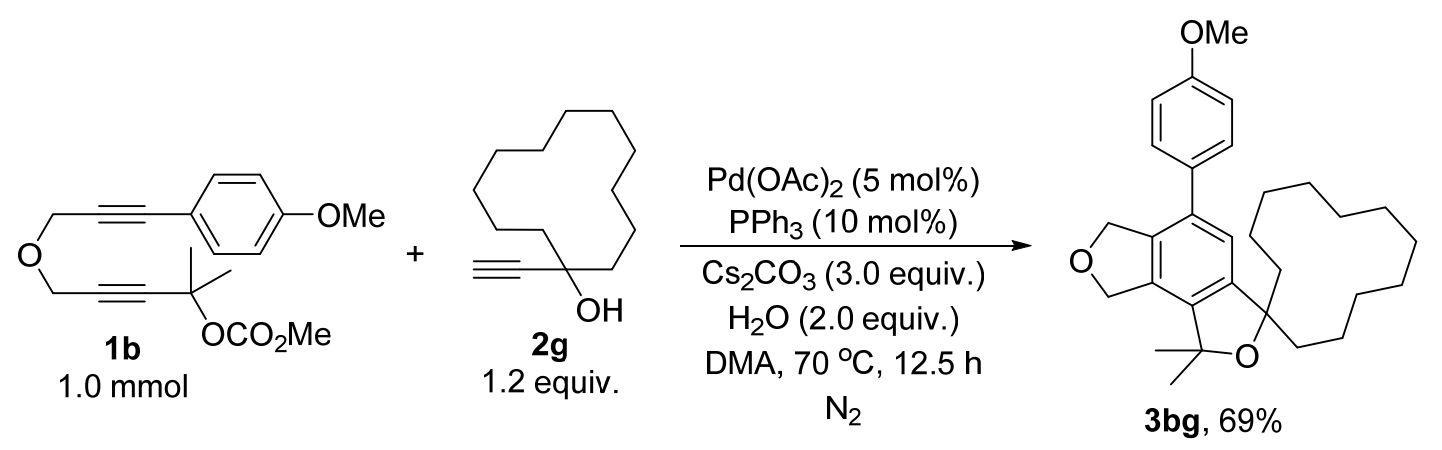

Following Typical Procedure, the reaction of $\mathrm{Cs}_{2} \mathrm{CO}_{3}(975.0 \mathrm{mg}, 3.0 \mathrm{mmol})$,

$\mathrm{Pd}(\mathrm{OAc})_{2}(11.8 \mathrm{mg}, 0.05 \mathrm{mmol}), \mathrm{PPh}_{3}(26.7 \mathrm{mg}, 0.1 \mathrm{mmol}), 2 \mathrm{~g}(250.8 \mathrm{mg}, 1.2 \mathrm{mmol})$,

1 b $(316.8 \mathrm{mg}, 1.0 \mathrm{mmol})$, and $\mathrm{H}_{2} \mathrm{O}(36.5 \mathrm{mg}, 2.0 \mathrm{mmol})$ in DMA (10 mL) afforded 3bg (311.1 mg, 69\%) (eluent: petroleum ether/ethyl acetate $=40 / 1(900 \mathrm{~mL})$ to $20 / 1$ $(300 \mathrm{~mL}))$ as a solid: m.p. 159.9-162.0 ${ }^{\circ} \mathrm{C}$ (n-hexane/DCM); ${ }^{1} \mathrm{H}$ NMR $(300 \mathrm{MHz}$, $\left.\mathrm{CDCl}_{3}\right) \delta 7.33(\mathrm{~d}, J=8.7 \mathrm{~Hz}, 2 \mathrm{H}, \mathrm{ArH}), 7.05(\mathrm{~s}, 1 \mathrm{H}, \mathrm{ArH}), 6.98(\mathrm{~d}, J=9.0 \mathrm{~Hz}, 2 \mathrm{H}$, $\mathrm{ArH})$, 5.22-5.13 (m, $\left.4 \mathrm{H}, \mathrm{OCH}_{2} \times 2\right), 3.86\left(\mathrm{~s}, 3 \mathrm{H}, \mathrm{CH}_{3}\right), 1.95-1.70\left(\mathrm{~m}, 4 \mathrm{H}, \mathrm{CH}_{2} \times 2\right)$, 1.69-1.54 (m, $\left.4 \mathrm{H}, \mathrm{CH}_{2} \times 2\right), 1.52\left(\mathrm{~s}, 6 \mathrm{H}, \mathrm{CH}_{3} \times 2\right), 1.48-1.25\left(\mathrm{~m}, 14 \mathrm{H}, \mathrm{CH}_{2} \times 7\right)$; ${ }^{13} \mathrm{C} \mathrm{NMR}\left(75 \mathrm{MHz}, \mathrm{CDCl}_{3}\right) \delta 159.0,146.7,138.2,137.0,134.7,132.7,132.3,129.0$ $121.1,114.0,89.2,83.3,73.2,71.3,55.3,36.6,29.1,26.5,26.0,22.7,22.2,19.9$; IR $(\mathrm{KBr}) \vee\left(\mathrm{cm}^{-1}\right) 3036,2940,2848,1610,1575,1517,1471,1443,1375,1359,1344$ $1285,1248,1177,1163,1142,1109,1053,1035,1001 ; \mathrm{MS}(\mathrm{EI}): \mathrm{m} / \mathrm{z}(\%) 448\left(\mathrm{M}^{+}\right.$, 84.06), 321 (100); Anal. Calcd. for $\mathrm{C}_{30} \mathrm{H}_{40} \mathrm{O}_{3}$ (\%): C 80.31, H 8.99, Found: C 80.47, H 8.96.

8. Synthesis of 1,1-dimethyl-5-(p-tolyl)-6,8-dihydro- $1 H$-spiro[benzo[1,2-c:3,4-c']difuran-3,1'-cyclohexane] (3ch). (zyc-1-181) 


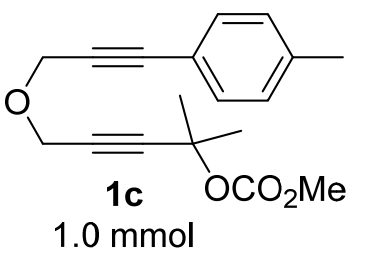

$1.0 \mathrm{mmol}$

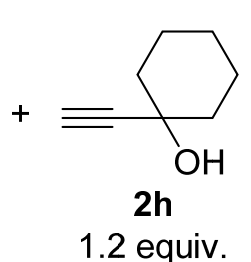

1.2 equiv.

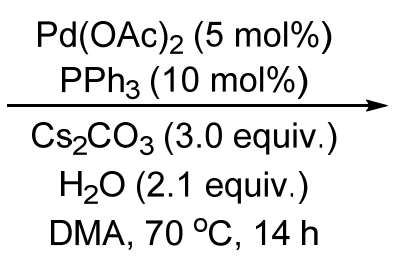

$\mathrm{N}_{2}$

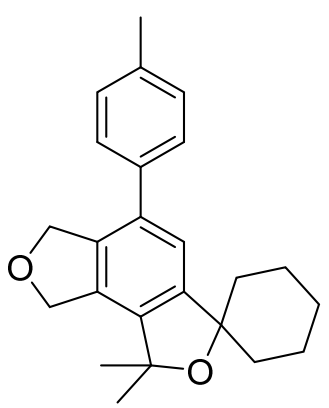

$3 \mathrm{ch}, 77 \%$

Following Typical Procedure, the reaction of $\mathrm{Cs}_{2} \mathrm{CO}_{3}(976.1 \mathrm{mg}, 3.0 \mathrm{mmol})$,

$\mathrm{Pd}(\mathrm{OAc})_{2}(11.8 \mathrm{mg}, 0.05 \mathrm{mmol}), \mathrm{PPh}_{3}(26.5 \mathrm{mg}, 0.1 \mathrm{mmol}), 2 \mathbf{h}(149.7 \mathrm{mg}, 1.2 \mathrm{mmol})$, 1c $(300.9 \mathrm{mg}, 1.0 \mathrm{mmol})$, and $\mathrm{H}_{2} \mathrm{O}(38.2 \mathrm{mg}, 2.1 \mathrm{mmol})$ in DMA $(10 \mathrm{~mL})$ afforded 3ch $(268.3 \mathrm{mg}, 77 \%)$ (eluent: petroleum ether/ethyl acetate $=20 / 1)$ as a solid: m.p. 164.7-165.7 ${ }^{\circ} \mathrm{C}$ (n-hexane/DCM); ${ }^{1} \mathrm{H}$ NMR $\left(300 \mathrm{MHz}, \mathrm{CDCl}_{3}\right) \delta 7.30(\mathrm{~d}, J=8.1 \mathrm{~Hz}$, $2 \mathrm{H}, \mathrm{ArH}), 7.24(\mathrm{~d}, J=8.1 \mathrm{~Hz}, 2 \mathrm{H}, \mathrm{ArH}), 7.03(\mathrm{~s}, 1 \mathrm{H}, \mathrm{ArH}), 5.18\left(\mathrm{~s}, 2 \mathrm{H}, \mathrm{OCH}_{2}\right)$, $5.17\left(\mathrm{~s}, 2 \mathrm{H}, \mathrm{OCH}_{2}\right), 2.39\left(\mathrm{~s}, 3 \mathrm{H}, \mathrm{CH}_{3}\right), 1.87-1.60\left(\mathrm{~m}, 9 \mathrm{H}, \mathrm{CH}_{2} \times 4\right.$ and one proton of $\left.\mathrm{CH}_{2}\right), 1.53\left(\mathrm{~s}, 6 \mathrm{H}, \mathrm{CH}_{3} \times 2\right), 1.40-1.23\left(\mathrm{~m}, 1 \mathrm{H}\right.$, one proton of $\left.\mathrm{CH}_{2}\right) ;{ }^{13} \mathrm{C}$ NMR $(75$ $\left.\mathrm{MHz}, \mathrm{CDCl}_{3}\right) \delta 147.2,138.6,137.3,137.21,137.15,135.3,132.3,129.3,127.7,120.5$, 85.7, 83.3, 73.1, 71.2, 39.6, 29.1, 25.2, 22.3, 21.1; IR (KBr) $v\left(\mathrm{~cm}^{-1}\right)$ 3024, 2962, 2929, $2889,2846,1616,1519,1473,1457,1437,1396,1374,1356,1334,1280,1185,1166$, 1134, 1090, 1059, 1032, 1020; MS (EI): m/z (\%) 348 (M+, 32.07), 305 (100); Anal. Calcd. for $\mathrm{C}_{24} \mathrm{H}_{28} \mathrm{O}_{2}$ (\%): C 82.72, H 8.10, Found: C 82.78, H 8.02.

9. Synthesis of 1-ethyl-1,3,3-trimethyl-5-(m-tolyl)-1,3,6,8-tetrahydrobenzo[1,2-c:3,4 $\left.-c^{\prime}\right]$ difuran (3da). (zyc-1-195) 

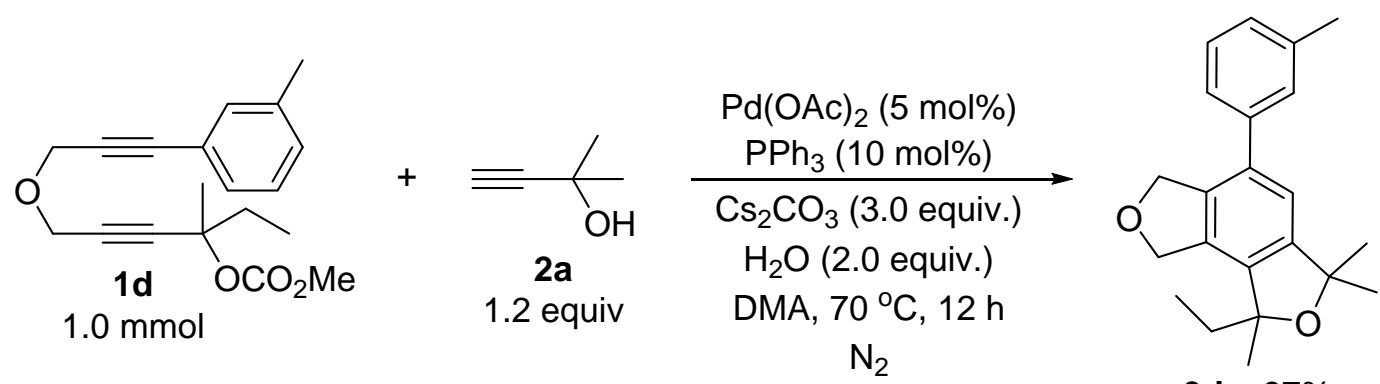

3da, $87 \%$

Following Typical Procedure, the reaction of $\mathrm{Cs}_{2} \mathrm{CO}_{3}(975.8 \mathrm{mg}, 3.0 \mathrm{mmol})$,

$\mathrm{Pd}(\mathrm{OAc})_{2}(11.6 \mathrm{mg}, 0.05 \mathrm{mmol}), \mathrm{PPh}_{3}(26.5 \mathrm{mg}, 0.1 \mathrm{mmol}), 2 \mathrm{a}(103.2 \mathrm{mg}, 1.2 \mathrm{mmol})$,

1d $(313.6 \mathrm{mg}, 1.0 \mathrm{mmol})$, and $\mathrm{H}_{2} \mathrm{O}(36.6 \mathrm{mg}, 2.0 \mathrm{mmol})$ in DMA $(10 \mathrm{~mL})$ afforded 3da $(281.1 \mathrm{mg}, 87 \%)$ (eluent: petroleum ether/ethyl acetate $=25 / 1)$ as a liquid: ${ }^{1} \mathrm{H}$ NMR (300 MHz, $\left.\mathrm{CDCl}_{3}\right) \delta$ 7.33-7.08 (m, $\left.4 \mathrm{H}, \mathrm{ArH}\right), 7.03$ (s, $\left.1 \mathrm{H}, \mathrm{ArH}\right)$, 5.24-5.05 (m, $\left.4 \mathrm{H}, \mathrm{OCH}_{2} \times 2\right), 2.38\left(\mathrm{~s}, 3 \mathrm{H}, \mathrm{CH}_{3}\right), 1.92-1.68\left(\mathrm{~m}, 2 \mathrm{H}, \mathrm{CH}_{2}\right), 1.57\left(\mathrm{~s}, 6 \mathrm{H}, \mathrm{CH}_{3} \times 2\right)$, $1.50\left(\mathrm{~s}, 3 \mathrm{H}, \mathrm{CH}_{3}\right), 0.83\left(\mathrm{t}, J=7.4 \mathrm{~Hz}, 3 \mathrm{H}, \mathrm{CH}_{3}\right) ;{ }^{13} \mathrm{C} \mathrm{NMR}\left(75 \mathrm{MHz}, \mathrm{CDCl}_{3}\right) \delta 147.4$, $139.9,138.0,136.9,136.6,135.6,132.1,128.4,128.3,127.9,124.7,120.0,86.5,84.3$, 72.9, 71.1, 32.7, 31.2, 29.7, 27.7, 21.2, 8.6; IR (neat) $v\left(\mathrm{~cm}^{-1}\right) 3025,2971,2923,2852$, $1606,1583,1475,1463,1448,1376,1362,1359,1343,1296,1263,1252,1179,1161$, 1145, 1109, 1092, 1058, 1028; MS (EI): m/z (\%) $322\left(\mathrm{M}^{+}, 0.15\right), 307\left(\left(\mathrm{M}_{-} \mathrm{CH}_{3}\right)^{+}\right.$, 17.18), $293\left(\left(\mathrm{M}-\mathrm{C}_{2} \mathrm{H}_{5}\right)^{+}, 100\right)$; HRMS calcd. for $\mathrm{C}_{22} \mathrm{H}_{26} \mathrm{O}_{2}\left[\mathrm{M}^{+}\right]$: 322.1933; Found: 322.1935 .

10. Synthesis of 5-(4-chlorophenyl)-1,1-dimethyl-6,8-dihydro-1H-spiro[benzo[1,2-c: 3,4-c']difuran-3,1'-cyclohexane] (3eh). (zyc-1-182) 


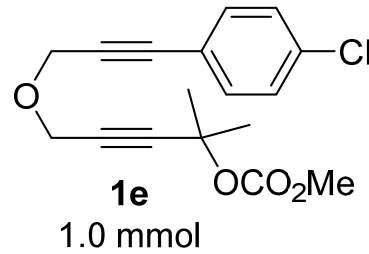

$1.0 \mathrm{mmol}$

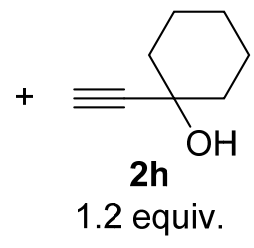

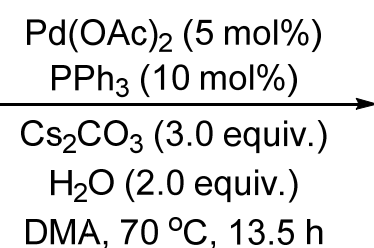

$\mathrm{N}_{2}$

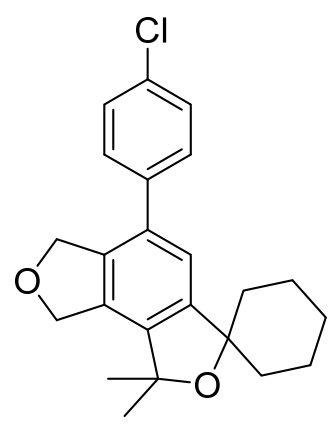

3eh, $77 \%$

Following Typical Procedure I, the reaction of $\mathrm{Cs}_{2} \mathrm{CO}_{3}(975.6 \mathrm{mg}, 3.0 \mathrm{mmol}$ ),

$\mathrm{Pd}(\mathrm{OAc})_{2}(11.8 \mathrm{mg}, 0.05 \mathrm{mmol}), \mathrm{PPh}_{3}(26.5 \mathrm{mg}, 0.1 \mathrm{mmol}), 2 \mathbf{h}(149.5 \mathrm{mg}, 1.2 \mathrm{mmol})$, 1e $(320.9 \mathrm{mg}, 1.0 \mathrm{mmol})$, and $\mathrm{H}_{2} \mathrm{O}(36.9 \mathrm{mg}, 2.0 \mathrm{mmol})$ in DMA $(10 \mathrm{~mL})$ afforded 3eh $(283.1 \mathrm{mg}, 77 \%)$ (eluent: petroleum ether/ethyl acetate $=30 / 1)$ as a solid: m.p. 197.8-199.0 ${ }^{\circ} \mathrm{C}\left(\right.$ n-hexane/DCM); ${ }^{1} \mathrm{H}$ NMR $\left(300 \mathrm{MHz}, \mathrm{CDCl}_{3}\right) \delta 7.41(\mathrm{~d}, J=8.7 \mathrm{~Hz}$, $2 \mathrm{H}, \mathrm{ArH}), 7.32$ (d, $J=8.7 \mathrm{~Hz}, 2 \mathrm{H}, \mathrm{ArH}), 7.00$ (s, $1 \mathrm{H}, \mathrm{ArH}), 5.23-5.16(\mathrm{~m}, 2 \mathrm{H}$, $\left.\mathrm{OCH}_{2}\right), 5.16-5.09\left(\mathrm{~m}, 2 \mathrm{H}, \mathrm{OCH}_{2}\right), 1.85-1.62\left(\mathrm{~m}, 9 \mathrm{H}, \mathrm{CH}_{2} \times 4\right.$ and one proton of $\left.\mathrm{CH}_{2}\right), 1.53\left(\mathrm{~s}, 6 \mathrm{H}, \mathrm{CH}_{3} \times 2\right), 1.40-1.22\left(\mathrm{~m}, 1 \mathrm{H}\right.$, one proton of $\left.\mathrm{CH}_{2}\right) ;{ }^{13} \mathrm{C}$ NMR $(75$ $\left.\mathrm{MHz}, \mathrm{CDCl}_{3}\right) \delta 147.4,139.3,138.7,137.3,134.2,133.5,132.6,129.2,128.8,120.5$ 85.7, 83.4, 73.0, 71.2, 39.6, 29.1, 25.2, 22.3; IR (KBr) $v\left(\mathrm{~cm}^{-1}\right)$ 2965, 2930, 2886, $2847,1498,1469,1443,1391,1374,1355,1347,1280,1212,1178,1166,1144,1134$, 1059, 1013; MS (EI): m/z (\%) $370\left(\mathrm{M}\left({ }^{37} \mathrm{Cl}\right)^{+}, 9.39\right), 368\left(\mathrm{M}\left({ }^{35} \mathrm{Cl}\right)^{+}, 30.47\right), 325(100)$; Anal. Calcd. for $\mathrm{C}_{23} \mathrm{H}_{25} \mathrm{ClO}_{2}$ (\%): C 74.89, H 6.83, Found: C 74.89, H 6.87.

11. Synthesis of 1,1-dimethyl-5-(thiophen-3-yl)-6,8-dihydro-1H-spiro[benzo[1,2-c: 3,4-c']difuran-3,1'-cyclohexane] (3fh). (zyc-1-192) 


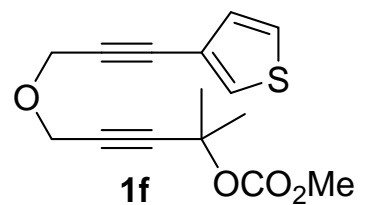

$1.0 \mathrm{mmol}$

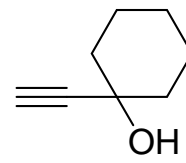

$2 \mathrm{~h}$

1.2 equiv.

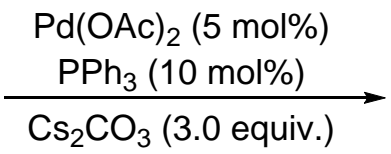

$\mathrm{H}_{2} \mathrm{O}$ (2.0 equiv.)

DMA, $70{ }^{\circ} \mathrm{C}, 12 \mathrm{~h}$

$\mathrm{N}_{2}$

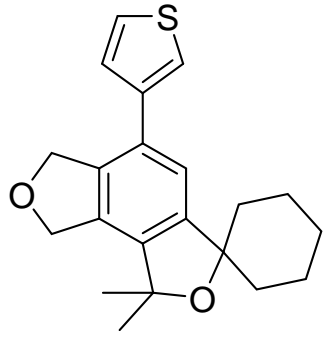

3fh, $78 \%$

Following Typical Procedure, the reaction of $\mathrm{Cs}_{2} \mathrm{CO}_{3}(976.2 \mathrm{mg}, 3.0 \mathrm{mmol})$,

$\mathrm{Pd}(\mathrm{OAc})_{2}(11.8 \mathrm{mg}, 0.05 \mathrm{mmol}), \mathrm{PPh}_{3}(26.8 \mathrm{mg}, 0.1 \mathrm{mmol}), 2 \mathrm{~h}(149.6 \mathrm{mg}, 1.2 \mathrm{mmol})$,

1f $(290.5 \mathrm{mg}, 0.99 \mathrm{mmol})$, and $\mathrm{H}_{2} \mathrm{O}(36.5 \mathrm{mg}, 2.0 \mathrm{mmol})$ in DMA (10 mL) afforded 3fh $(264.4 \mathrm{mg}, 78 \%)$ (eluent: petroleum ether/ethyl acetate $=25 / 1)$ as a solid: m.p. 121.5-122. $3{ }^{\circ} \mathrm{C}$ (n-hexane/DCM); ${ }^{1} \mathrm{H}$ NMR $\left(300 \mathrm{MHz}, \mathrm{CDCl}_{3}\right) \delta$ 7.44-7.38 (m, $1 \mathrm{H}$, ArH), 7.30-7.23 (m, $2 \mathrm{H}, \mathrm{ArH}), 7.13(\mathrm{~s}, 1 \mathrm{H}, \mathrm{ArH})$, 5.25-5.16 (m, $\left.4 \mathrm{H}, \mathrm{OCH}_{2} \times 2\right)$, 1.90-1.60 (m, $9 \mathrm{H}, \mathrm{CH}_{2} \times 4$ and one proton of $\left.\mathrm{CH}_{2}\right), 1.52\left(\mathrm{~s}, 6 \mathrm{H}, \mathrm{CH}_{3} \times 2\right), 1.44-1.22$ (m, $1 \mathrm{H}$, one proton of $\left.\mathrm{CH}_{2}\right) ;{ }^{13} \mathrm{C}$ NMR $\left(75 \mathrm{MHz}, \mathrm{CDCl}_{3}\right) \delta 147.2,140.6,138.8,136.9$, $132.5,129.8,127.1,126.0,121.7,119.8,85.6,83.3,73.5,71.2,39.6,29.1,25.2,22.3$; IR $(\mathrm{KBr}) \vee\left(\mathrm{cm}^{-1}\right) 2973,2923,2859,1474,1457,1443,1423,1376,1358,1314,1281$, 1164, 1134, 1052, 1032; MS (EI): m/z (\%) 340 (M+1 40.85), 297 (100); Anal. Calcd. for $\mathrm{C}_{21} \mathrm{H}_{24} \mathrm{O}_{2} \mathrm{~S}(\%)$ : C 74.08, H 7.11, Found: C 74.06, H 7.08.

12. Synthesis of 1,3,3-trimethyl-5-phenyl-1-propyl-1,3,6,8-tetrahydrobenzo[1,2-c:3,4 $-c^{\prime}$ ]difuran (3ga). (zyc-2-22)
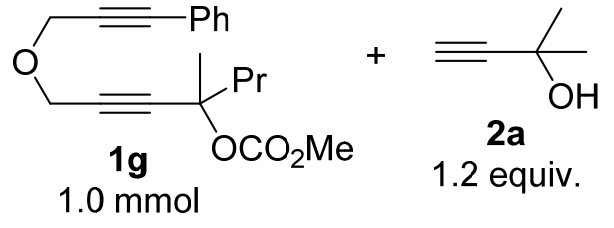

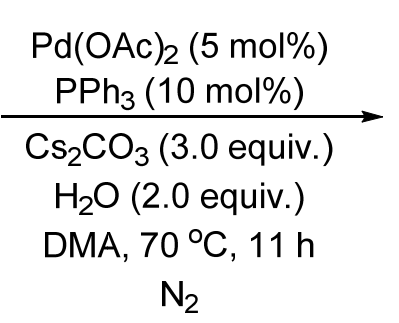

S18

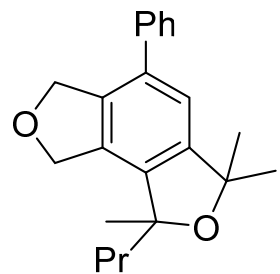

3ga, $81 \%$ 
Following Typical Procedure, the reaction of $\mathrm{Cs}_{2} \mathrm{CO}_{3}(976.3 \mathrm{mg}, 3.0 \mathrm{mmol}$ ), $\mathrm{Pd}(\mathrm{OAc})_{2}(11.1 \mathrm{mg}, 0.05 \mathrm{mmol}), \mathrm{PPh}_{3}(26.5 \mathrm{mg}, 0.1 \mathrm{mmol}), 2 \mathrm{a}(103.9 \mathrm{mg}, 1.2 \mathrm{mmol})$, $1 \mathrm{~g}(312.9 \mathrm{mg}, 1.0 \mathrm{mmol})$, and $\mathrm{H}_{2} \mathrm{O}(36.1 \mathrm{mg}, 2.0 \mathrm{mmol})$ in DMA (10 mL) afforded 3ga (260.9 mg, 81\%) (eluent: petroleum ether/ethyl acetate $=50 / 1)$ as a solid; m.p. 92.9-93.9 ${ }^{\circ} \mathrm{C}$ (n-hexane/DCM); ${ }^{1} \mathrm{H}$ NMR $\left(300 \mathrm{MHz}, \mathrm{CDCl}_{3}\right) \delta$ 7.48-7.32 (m, $5 \mathrm{H}$, ArH), $7.02(\mathrm{~s}, 1 \mathrm{H}, \mathrm{ArH}), 5.22-5.06\left(\mathrm{~m}, 4 \mathrm{H}, \mathrm{OCH}_{2} \times 2\right), 1.88-1.63\left(\mathrm{~m}, 2 \mathrm{H}, \mathrm{CH}_{2}\right)$, $1.56\left(\mathrm{~s}, 6 \mathrm{H}, \mathrm{CH}_{3} \times 2\right), 1.50\left(\mathrm{~s}, 3 \mathrm{H}, \mathrm{CH}_{3}\right), 1.48-1.33\left(\mathrm{~m}, 1 \mathrm{H}\right.$, one proton of $\left.\mathrm{CH}_{2}\right)$, 1.15-0.96 (m, $1 \mathrm{H}$, one proton of $\left.\mathrm{CH}_{2}\right), 0.89\left(\mathrm{t}, J=7.2 \mathrm{~Hz}, 3 \mathrm{H}, \mathrm{CH}_{3}\right) ;{ }^{13} \mathrm{C}$ NMR $(75$ $\left.\mathrm{MHz}, \mathrm{CDCl}_{3}\right) \delta 147.5,140.2,137.3,137.1,135.6,132.3,128.6,127.8,127.4,120.3$, 86.5, 84.5, 73.1, 71.4, 42.7, 31.4, 29.9, 28.2, 17.8, 14.3; IR (neat) $v\left(\mathrm{~cm}^{-1}\right) 3062,3024$, 2966, 2926, 2870, 1598, 1573, 1472, 1450, 1377, 1359, 1342, 1289, 1272, 1249, 1231, 1175, 1148, 1128, 1057, 1029; MS (EI): m/z (\%) $322\left(\mathrm{M}^{+}, 0.05\right), 307\left(\left(\mathrm{M}-\mathrm{CH}_{3}\right)^{+}\right.$,

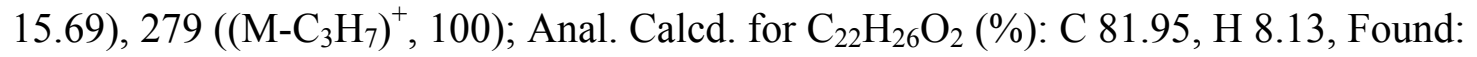
C 81.86, H 8.08.

13.

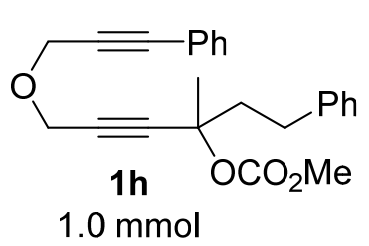

$1.0 \mathrm{mmol}$

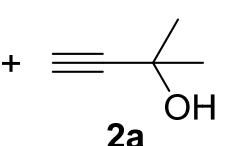

2a 1.2 equiv.

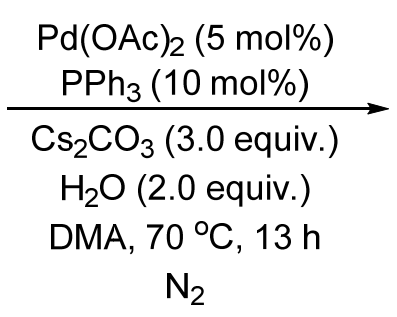

$\mathrm{N}_{2}$

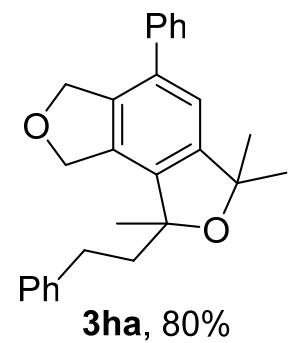

3ha, $80 \%$

Following Typical Procedure, the reaction of $\mathrm{Cs}_{2} \mathrm{CO}_{3}(976.3 \mathrm{mg}, 3.0 \mathrm{mmol})$, 
$\mathrm{Pd}(\mathrm{OAc})_{2}(11.7 \mathrm{mg}, 0.05 \mathrm{mmol}), \mathrm{PPh}_{3}(27.3 \mathrm{mg}, 0.1 \mathrm{mmol}), 2 \mathrm{a}(103.3 \mathrm{mg}, 1.2 \mathrm{mmol})$, 1h $(375.3 \mathrm{mg}, 1.0 \mathrm{mmol})$, and $\mathrm{H}_{2} \mathrm{O}(35.2 \mathrm{mg}, 2.0 \mathrm{mmol})$ in DMA (10 mL) afforded 3ha $(305.3 \mathrm{mg}, 80 \%)($ eluent: petroleum ether/ethyl acetate/ethyl ether $=50 / 1 / 1)$ as a solid: m.p. 97.9-98.5 ${ }^{\circ} \mathrm{C}$ (n-hexane/DCM); ${ }^{1} \mathrm{H}$ NMR (300 MHz, $\left.\mathrm{CDCl}_{3}\right) \delta$ 7.48-7.29 (m, 5 H, ArH), 7.28-7.18 (m, 2 H, ArH), 7.18-7.08 (m, 3 H, ArH), 7.06 (s, 1 H, ArH), 5.24-5.06 (m, $\left.4 \mathrm{H}, \mathrm{OCH}_{2} \times 2\right), 2.76\left(\mathrm{td}, J_{1}=12.8 \mathrm{~Hz}, J_{2}=4.6 \mathrm{~Hz}, 1 \mathrm{H}\right.$, one proton of $\left.\mathrm{CH}_{2}\right), 2.32\left(\mathrm{td}, J_{1}=12.8 \mathrm{~Hz}, J_{2}=4.5 \mathrm{~Hz}, 1 \mathrm{H}\right.$, one proton of $\left.\mathrm{CH}_{2}\right), 2.16\left(\mathrm{td}, J_{1}=13.1\right.$ $\mathrm{Hz}, J_{2}=4.9 \mathrm{~Hz}, 1 \mathrm{H}$, one proton of $\left.\mathrm{CH}_{2}\right), 2.00\left(\mathrm{td}, J_{1}=13.1 \mathrm{~Hz}, J_{2}=4.4 \mathrm{~Hz}, 1 \mathrm{H}\right.$, one proton of $\left.\mathrm{CH}_{2}\right), 1.64\left(\mathrm{~s}, 3 \mathrm{H}, \mathrm{CH}_{3}\right), 1.61\left(\mathrm{~s}, 3 \mathrm{H}, \mathrm{CH}_{3}\right), 1.55\left(\mathrm{~s}, 3 \mathrm{H}, \mathrm{CH}_{3}\right) ;{ }^{13} \mathrm{C} \mathrm{NMR}$ $\left(75 \mathrm{MHz}, \mathrm{CDCl}_{3}\right) \delta 147.5,142.2,140.0,137.2,136.6,135.8,132.3,128.6,128.3$, $127.8,127.4,125.6,120.3,86.2,84.7,73.1,71.3,42.5,31.4,31.1,29.9,28.2$; IR (KBr) $v\left(\mathrm{~cm}^{-1}\right) 3084,3060,3026,2970,2925,2857,1602,1575,1497,1473,1453,1403$, 1369, 1359, 1343, 1300, 1244, 1181, 1148, 1104, 1086, 1056, 1029; MS (EI): m/z (\%) $384\left(\mathrm{M}^{+}, 1.92\right), 280$ (100); Anal. Calcd. for $\mathrm{C}_{27} \mathrm{H}_{28} \mathrm{O}_{2}$ (\%): C 84.34, H 7.34, Found: $\mathrm{C}$ 84.06, H 7.28.

14. Synthesis of 1,1-diethyl-3,3-dimethyl-5-phenyl-1,3,6,8-tetrahydrobenzo[1,2-c:3,4 $\left.-c^{\prime}\right]$ difuran (3ia). (zyc-2-18)

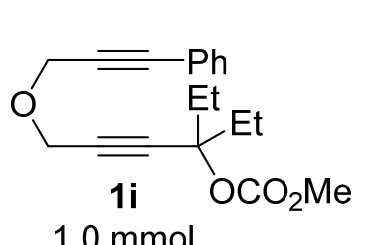

$1.0 \mathrm{mmol}$

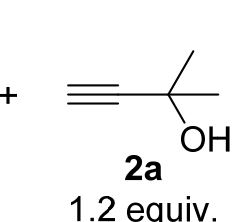

1.2 equiv.

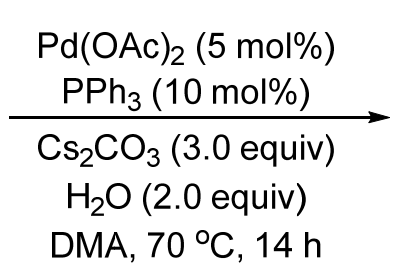

$\mathrm{N}_{2}$

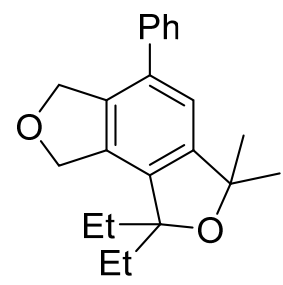

3ia, $76 \%$

Following Typical Procedure, the reaction of $\mathrm{Cs}_{2} \mathrm{CO}_{3}(976.3 \mathrm{mg}, 3.0 \mathrm{mmol})$, 
$\mathrm{Pd}(\mathrm{OAc})_{2}(11.4 \mathrm{mg}, 0.05 \mathrm{mmol}), \mathrm{PPh}_{3}(26.9 \mathrm{mg}, 0.1 \mathrm{mmol}), 2 \mathrm{a}(103.8 \mathrm{mg}, 1.2 \mathrm{mmol})$, 1i $(314.8 \mathrm{mg}, 1.0 \mathrm{mmol})$, and $\mathrm{H}_{2} \mathrm{O}(36.2 \mathrm{mg}, 2.0 \mathrm{mmol})$ in DMA $(10 \mathrm{~mL})$ afforded 3ia (245.3 mg, 76\%) (eluent: petroleum ether/ethyl acetate/ethyl ether $=100 / 2 / 1)$ as a solid: m.p. 84.7-85.5 ${ }^{\circ} \mathrm{C}$ (n-hexane/DCM); ${ }^{1} \mathrm{H}$ NMR (300 MHz, $\left.\mathrm{CDCl}_{3}\right) \delta$ 7.48-7.30 (m, $5 \mathrm{H}, \mathrm{ArH}), 7.02(\mathrm{~s}, 1 \mathrm{H}, \mathrm{ArH}), 5.20-5.14\left(\mathrm{~m}, 2 \mathrm{H}, \mathrm{OCH}_{2}\right)$, 5.14-5.07 (m, $2 \mathrm{H}$, $\left.\mathrm{OCH}_{2}\right), 1.98-1.82\left(\mathrm{~m}, 2 \mathrm{H}, \mathrm{CH}_{2}\right), 1.76-1.61\left(\mathrm{~m}, 2 \mathrm{H}, \mathrm{CH}_{2}\right), 1.58\left(\mathrm{~s}, 6 \mathrm{H}, \mathrm{CH}_{3} \times 2\right)$, $0.87\left(\mathrm{t}, J=7.4 \mathrm{~Hz}, 6 \mathrm{H}, \mathrm{CH}_{3} \times 2\right) ;{ }^{13} \mathrm{C} \mathrm{NMR}\left(75 \mathrm{MHz}, \mathrm{CDCl}_{3}\right) \delta$ 148.0, 140.2, 136.9, $136.3,135.5,132.4,128.6,127.8,127.4,120.2,89.9,84.4,73.1,71.6,31.6,30.5,8.6$; IR $(\mathrm{KBr}) \vee\left(\mathrm{cm}^{-1}\right) 3054,2976,2934,2916,2877,2854,1468,1456,1450,1437,1402$, 1376, 1356, 1334, 1286, 1250, 1178, 1157, 1144, 1116, 1055, 1029; MS (EI): m/z (\%)

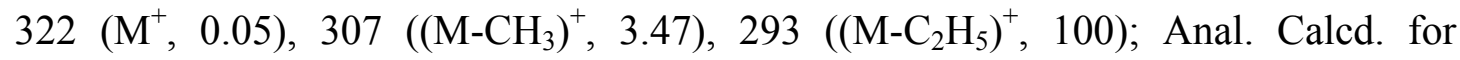
$\mathrm{C}_{22} \mathrm{H}_{26} \mathrm{O}_{2}(\%)$ : C 81.95, H 8.13, Found: C 81.90, H 8.06.

15. Synthesis of 3,3-dimethyl-5-phenyl-6,8-dihydro-3H-spiro[benzo[1,2-c:3,4-c']difuran-1,1'-cyclopentane] (3ja). (zyc-2-11)
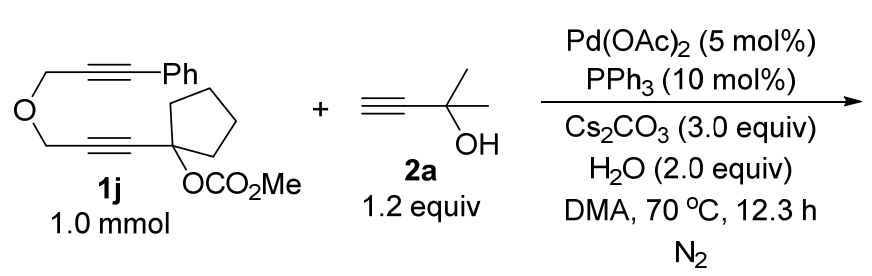

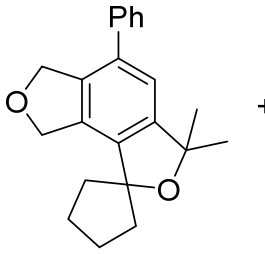

3ja, 44\% before separation: $3 \mathbf{j a}, 45 \%$; $\mathbf{4 j a}, 10 \%$

Following Typical Procedure, the reaction of $\mathrm{Cs}_{2} \mathrm{CO}_{3}(976.7 \mathrm{mg}, 3.0 \mathrm{mmol})$, $\mathrm{Pd}(\mathrm{OAc})_{2}(11.8 \mathrm{mg}, 0.05 \mathrm{mmol}), \mathrm{PPh}_{3}(27.0 \mathrm{mg}, 0.1 \mathrm{mmol}), 2 \mathrm{a}(103.2 \mathrm{mg}, 1.2 \mathrm{mmol})$, 1j (312.4 mg, $1.0 \mathrm{mmol})$, and $\mathrm{H}_{2} \mathrm{O}(36.2 \mathrm{mg}, 2.0 \mathrm{mmol})$ in DMA (10 mL) afforded 3ja $(142.1 \mathrm{mg}, 44 \%)$ and $\mathbf{4 j a}(33.2 \mathrm{mg}$, purity $=95 \%, 10 \%)$ (The crude product was 
firstly purified by chromatography on silica gel with the eluent (petroleum ether/ethyl acetate $=20 / 1(500 \mathrm{~mL})$ to $15 / 1(600 \mathrm{~mL}))$ to afford pure $3 \mathbf{j a}$ and impure $\mathbf{4 j a}$, which was further purified by chromatography on silica gel with the eluent (petroleum ether/ethyl acetate $=20 / 1)$ ).

3ja: solid; m.p. $125.6-127.4{ }^{\circ} \mathrm{C}$ (n-hexane/DCM); ${ }^{1} \mathrm{H}$ NMR $\left(300 \mathrm{MHz}, \mathrm{CDCl}_{3}\right) \delta$ 7.49-7.33 (m, $5 \mathrm{H}, \mathrm{ArH}), 7.04(\mathrm{~s}, 1 \mathrm{H}, \mathrm{ArH}), 5.16\left(\mathrm{~s}, 4 \mathrm{H}, \mathrm{OCH}_{2} \times 2\right), 2.05-1.87(\mathrm{~m}, 6$ $\left.\mathrm{H}, \mathrm{CH}_{2} \times 3\right), 1.86-1.70\left(\mathrm{~m}, 2 \mathrm{H}, \mathrm{CH}_{2}\right), 1.54\left(\mathrm{~s}, 6 \mathrm{H}, \mathrm{CH}_{3} \times 2\right) ;{ }^{13} \mathrm{C} \mathrm{NMR}(75 \mathrm{MHz}$, $\left.\mathrm{CDCl}_{3}\right) \delta 148.0,140.2,137.4,136.9,135.6,132.5,128.7,127.9,127.5,120.1,93.5$ 84.4, 73.1, 71.2, 40.6, 30.7, 25.4; IR (neat) $v\left(\mathrm{~cm}^{-1}\right)$ 3058, 3029, 2967, 2867, 1602, $1575,1502,1471,1449,1433,1404,1377,1359,1347,1316,1266,1244,1185,1153$, 1120, 1074, 1054, 1030; MS (EI): m/z (\%) 320 (M+, 72.57), 291 (100); Anal. Calcd. for $\mathrm{C}_{22} \mathrm{H}_{24} \mathrm{O}_{2}(\%)$ : C 82.46, H 7.55, Found: C 82.36, H 7.38.

4ja: liquid; ${ }^{1} \mathrm{H} \mathrm{NMR}\left(300 \mathrm{MHz}, \mathrm{CDCl}_{3}\right) \delta$ 7.50-7.30 (m, $\left.6 \mathrm{H}, \mathrm{ArH}\right)$, 5.74-5.66 (m, $1 \mathrm{H},=\mathrm{CH})$, 5.25-5.19 (m, $\left.2 \mathrm{H}, \mathrm{OCH}_{2}\right)$, 5.04-4.96 (m, $\left.2 \mathrm{H}, \mathrm{OCH}_{2}\right), 2.82(\mathrm{~s}, 1 \mathrm{H}, \mathrm{OH})$, 2.73-2.60 (m, $\left.2 \mathrm{H}, \mathrm{CH}_{2}\right), 2.58-2.48\left(\mathrm{~m}, 2 \mathrm{H}, \mathrm{CH}_{2}\right), 2.13-1.97\left(\mathrm{~m}, 2 \mathrm{H}, \mathrm{CH}_{2}\right), 1.65$ (s, 6 $\left.\mathrm{H}, \mathrm{CH}_{3} \times 2\right) ;{ }^{13} \mathrm{C} \mathrm{NMR}\left(75 \mathrm{MHz}, \mathrm{CDCl}_{3}\right) \delta 146.8,144.3,140.1,140.0,135.0,134.2$, $129.8,129.6,128.7,127.8,127.4,125.8,74.4,73.8,73.4,37.9,33.2,32.6,24.0$; IR (neat) $v\left(\mathrm{~cm}^{-1}\right) 3438,3054,3024,2968,2931,2849,1599,1471,1445,1361,1267$, 1237, 1165, 1124, 1075, 1050, 1040; MS (EI): m/z (\%) $320\left(\mathrm{M}^{+}, 4.81\right), 302$ (100); HRMS calcd. for $\mathrm{C}_{22} \mathrm{H}_{24} \mathrm{O}_{2}\left[\mathrm{M}^{+}\right]$: 320.1776 , Found: 320.1776 .

16. Synthesis of 5'-phenyl-6',8'-dihydrodispiro[cyclohexane-1,3'-benzo[1,2-c:3,4-c']d- 
ifuran-1',1"-cyclopentane] (3jh). (zyc-2-24)
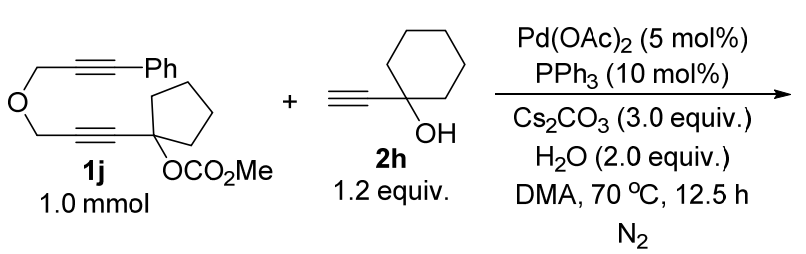

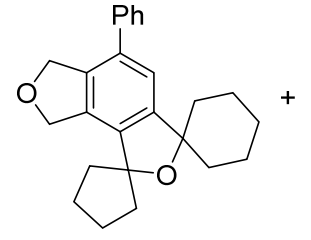

$3 \mathrm{jh}, 52 \%$

4jh, 6\%

Following Typical Procedure, the reaction of $\mathrm{Cs}_{2} \mathrm{CO}_{3}(976.3 \mathrm{mg}, 3.0 \mathrm{mmol})$, $\mathrm{Pd}(\mathrm{OAc})_{2}(11.2 \mathrm{mg}, 0.05 \mathrm{mmol}), \mathrm{PPh}_{3}(27.1 \mathrm{mg}, 0.1 \mathrm{mmol}), 2 \mathbf{h}(149.6 \mathrm{mg}, 1.2 \mathrm{mmol})$, 1j (313.6 mg, $1.0 \mathrm{mmol})$, and $\mathrm{H}_{2} \mathrm{O}(36.7 \mathrm{mg}, 2.0 \mathrm{mmol})$ in DMA (10 mL) afforded 3jh (189.7 mg, 52\%) and 4jh (22.9 mg, purity = 95\%, 6\%) (The crude product was first purified by chromatography on silica gel with the eluent (petroleum ether/ethyl acetate $=50 / 1$ to $20 / 1)$ to afford $3 \mathbf{j h}$ and impure $\mathbf{4 j h}$, which was further purified by chromatography on silica gel with the eluent (petroleum ether/DCM =2/1)).

3jh: solid; m.p. $162.4-164.6{ }^{\circ} \mathrm{C}$ (n-hexane/DCM); ${ }^{1} \mathrm{H}$ NMR $\left(300 \mathrm{MHz}, \mathrm{CDCl}_{3}\right) \delta$ 7.49-7.28 (m, $5 \mathrm{H}, \mathrm{ArH}), 7.04$ (s, $1 \mathrm{H}, \mathrm{ArH})$, 5.20-5.08 (m, $\left.4 \mathrm{H}, \mathrm{OCH}_{2} \times 2\right), 2.06-1.52$ (m, $17 \mathrm{H}, \mathrm{CH}_{2} \times 8$ and one proton of $\left.\mathrm{CH}_{2}\right), 1.40-1.18\left(\mathrm{~m}, 1 \mathrm{H}\right.$, one proton of $\mathrm{CH}_{2}$ ); ${ }^{13} \mathrm{C} \mathrm{NMR}\left(75 \mathrm{MHz}, \mathrm{CDCl}_{3}\right) \delta 148.4,140.4,137.4,137.3,135.2,132.5,128.6,127.9$, $127.4,120.3,92.9,85.2,73.0,71.2,40.7,39.3,25.4,25.3,22.4 ; \mathrm{IR}(\mathrm{KBr}) \vee\left(\mathrm{cm}^{-1}\right)$ $3080,3024,2933,2922,2866,2843,1465,1442,1427,1377,1347,1332,1263,1180$, 1154, 1132, 1094, 1071, 1052, 1029; MS (EI): m/z (\%) $360\left(\mathrm{M}^{+}, 75.52\right), 317(100)$; Anal. Calcd. for $\mathrm{C}_{25} \mathrm{H}_{28} \mathrm{O}_{2}$ (\%): C 83.29, H 7.83, Found: C 83.55, H 7.66.

4jh: liquid; ${ }^{1} \mathrm{H}$ NMR (300 MHz, $\left.\mathrm{CDCl}_{3}\right) \delta$ 7.50-7.30 (m, $\left.6 \mathrm{H}, \mathrm{ArH}\right)$, 5.70-5.62 (m, $1 \mathrm{H},=\mathrm{CH}), 5.25-5.17\left(\mathrm{~m}, 2 \mathrm{H}, \mathrm{OCH}_{2}\right), 5.04-4.96\left(\mathrm{~m}, 2 \mathrm{H}, \mathrm{OCH}_{2}\right), 2.67(\mathrm{~s}, 1 \mathrm{H}, \mathrm{OH})$, 2.58-2.47 (m, $3 \mathrm{H}, \mathrm{CH}_{2}$ and one proton of $\left.\mathrm{CH}_{2}\right), 2.14-1.66\left(\mathrm{~m}, 10 \mathrm{H}, \mathrm{CH}_{2} \times 5\right)$, 
1.65-1.53 (m, $\left.2 \mathrm{H}, \mathrm{CH}_{2}\right), 1.34-1.14\left(\mathrm{~m}, 1 \mathrm{H}\right.$, one proton of $\left.\mathrm{CH}_{2}\right) ;{ }^{13} \mathrm{C} \mathrm{NMR}(75 \mathrm{MHz}$, $\left.\mathrm{CDCl}_{3}\right) \delta 147.3,144.9,140.2,140.0,134.9,134.2,129.9,129.4,128.7,127.8,127.4$ 126.0, 75.5, 73.9, 73.5, 40.3, 38.8, 38.1, 33.2, 25.5, 24.0, 22.0; IR (neat) $v\left(\mathrm{~cm}^{-1}\right) 3448$, $3032,2927,2849,1605,1497,1465,1445,1361,1347,1258,1169,1147,1128,1059$, 1040; MS (EI): m/z (\%) $360\left(\mathrm{M}^{+}, 2.87\right), 342$ (100); HRMS calcd. for $\mathrm{C}_{25} \mathrm{H}_{28} \mathrm{O}_{2}\left[\mathrm{M}^{+}\right]$: 360.2089, Found: 360.2087.

17. Synthesis of 5-butyl-1,1,3,3-tetramethyl-1,3,6,8-tetrahydrobenzo[1,2-c:3,4-c']difuran (3ka). (zyc-3-77)
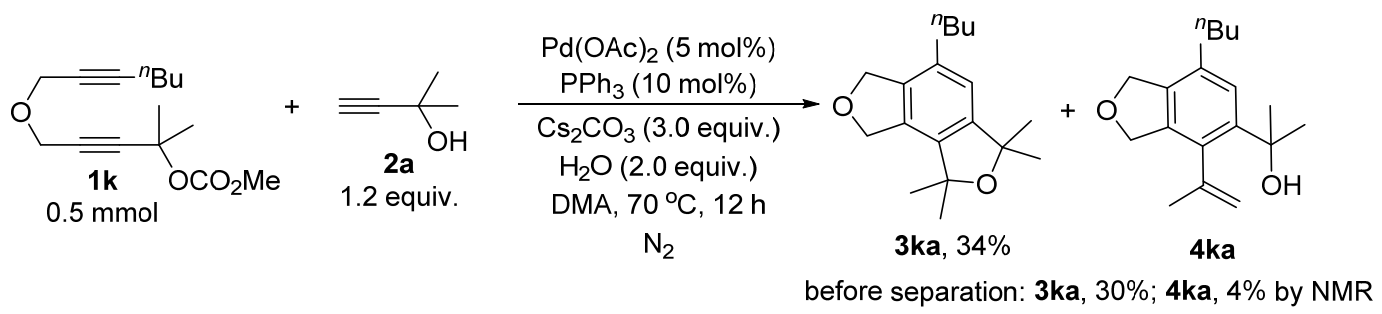

Following Typical Procedure, the reaction of $\mathrm{Cs}_{2} \mathrm{CO}_{3}(487.9 \mathrm{mg}, 1.5 \mathrm{mmol})$, $\mathrm{Pd}(\mathrm{OAc})_{2}(5.6 \mathrm{mg}, 0.025 \mathrm{mmol}), \mathrm{PPh}_{3}(13.1 \mathrm{mg}, 0.05 \mathrm{mmol}), 2 \mathrm{a}(50.5 \mathrm{mg}, 0.6 \mathrm{mmol})$, 1k (133.4 mg, $0.5 \mathrm{mmol})$, and $\mathrm{H}_{2} \mathrm{O}(18.0 \mu \mathrm{L}, \mathrm{d}=1.0 \mathrm{~g} / \mathrm{mL}, 18.0 \mathrm{mg}, 1.0 \mathrm{mmol})$ in DMA (5 mL) afforded 3ka (46.9 mg, 34\%) (eluent: petroleum ether/ethyl acetate = 30/1) as a liquid: ${ }^{1} \mathrm{H}$ NMR $\left(300 \mathrm{MHz}, \mathrm{CDCl}_{3}\right) \delta 6.80(\mathrm{~s}, 1 \mathrm{H}, \mathrm{ArH}), 5.18-5.12(\mathrm{~m}, 2 \mathrm{H}$, $\left.\mathrm{OCH}_{2}\right), 5.12-5.07$ (m, $\left.2 \mathrm{H}, \mathrm{OCH}_{2}\right), 2.58-2.47$ (m, $\left.2 \mathrm{H}, \mathrm{CH}_{2}\right), 1.64-1.53\left(\mathrm{~m}, 2 \mathrm{H}, \mathrm{CH}_{2}\right)$, $1.52\left(\mathrm{~s}, 6 \mathrm{H}, \mathrm{CH}_{3} \times 2\right), 1.48\left(\mathrm{~s}, 6 \mathrm{H}, \mathrm{CH}_{3} \times 2\right), 1.47-1.32\left(\mathrm{~m}, 2 \mathrm{H}, \mathrm{CH}_{2}\right), 0.95(\mathrm{t}, J=$ $\left.7.4 \mathrm{~Hz}, 3 \mathrm{H}, \mathrm{CH}_{3}\right) ;{ }^{13} \mathrm{C} \mathrm{NMR}\left(75 \mathrm{MHz}, \mathrm{CDCl}_{3}\right) \delta 146.6,137.6,136.8,135.8,131.3$, 119.8, 84.5, 83.6, 72.5, 71.5, 33.2, 32.2, 30.9, 28.9, 22.6, 13.9; IR (neat) $v\left(\mathrm{~cm}^{-1}\right) 2969$, 
2928, 2859, 1462, 1374, 1361, 1336, 1278, 1176, 1123, 1057; MS (EI): m/z (\%) 274 $\left(\mathrm{M}^{+}, 0.28\right), 259$ ([M-CH$\left.]^{+}, 100\right)$; HRMS calcd. for $\mathrm{C}_{18} \mathrm{H}_{26} \mathrm{O}_{2}\left[\mathrm{M}^{+}\right]$: 274.1933; Found: 274.1934.

4ak was not obtained by chromatography on silica gel.

18. Synthesis of 1,1,3,3-tetramethyl-5-phenyl-3,6,7,8-tetrahydro- $1 H$-indeno[4,5-c]furan (3la). (zyc-2-4)
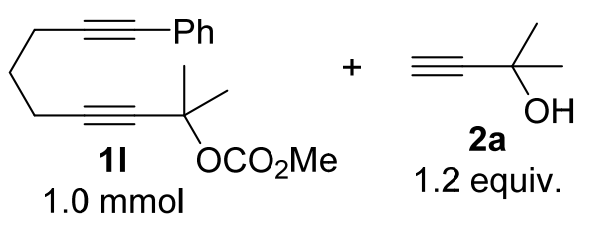

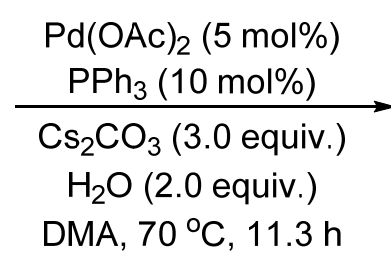

$\mathrm{N}_{2}$

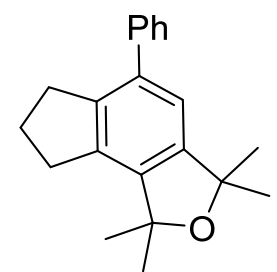

3la, $49 \%$

Following Typical Procedure, the reaction of $\mathrm{Cs}_{2} \mathrm{CO}_{3}(976.5 \mathrm{mg}, 3.0 \mathrm{mmol})$, $\mathrm{Pd}(\mathrm{OAc})_{2}(11.5 \mathrm{mg}, 0.05 \mathrm{mmol}), \mathrm{PPh}_{3}(26.9 \mathrm{mg}, 0.1 \mathrm{mmol}), 2 \mathrm{a}(103.9 \mathrm{mg}, 1.2 \mathrm{mmol})$, 11 (283.8 mg, $1.0 \mathrm{mmol})$, and $\mathrm{H}_{2} \mathrm{O}(36.8 \mathrm{mg}, 2.0 \mathrm{mmol})$ in DMA (10 mL) afforded 3la (142.4 mg, 49\%) (eluent: petroleum ether $(600 \mathrm{~mL})$ to petroleum ether/ethyl acetate $=$ 50/1 (500 mL)) as a solid: m.p. 94.8-95.5 ${ }^{\circ} \mathrm{C}$ (n-hexane/DCM); ${ }^{1} \mathrm{H}$ NMR $(300 \mathrm{MHz}$, $\left.\mathrm{CDCl}_{3}\right) \delta$ 7.48-7.27 (m, $\left.5 \mathrm{H}, \mathrm{ArH}\right), 6.92(\mathrm{~s}, 1 \mathrm{H}, \mathrm{ArH}), 2.96\left(\mathrm{q}, J=7.8 \mathrm{~Hz}, 4 \mathrm{H}, \mathrm{CH}_{2} \times\right.$ 2), 2.17-2.03 (m, $\left.2 \mathrm{H}, \mathrm{CH}_{2}\right), 1.59\left(\mathrm{~s}, 6 \mathrm{H}, \mathrm{CH}_{3} \times 2\right), 1.54\left(\mathrm{~s}, 6 \mathrm{H}, \mathrm{CH}_{3} \times 2\right) ;{ }^{13} \mathrm{C} \mathrm{NMR}$ $\left(75 \mathrm{MHz}, \mathrm{CDCl}_{3}\right) \delta 145.5,142.2,141.6,140.4,137.9,137.1,128.6,128.2,126.8$, 119.4, 84.1, 84.0, 32.2, 31.1, 30.2, 28.9, 25.9; IR (KBr) $v\left(\mathrm{~cm}^{-1}\right) 3058,3024,2973$, 2962, 2925, 1497, 1473, 1448, 1397, 1372, 1358, 1304, 1270, 1240, 1175, 1132, 1092, 1074, 1027; MS (EI): m/z (\%) $292\left(\mathrm{M}^{+}, 1.88\right), 277$ (100); Anal. Calcd. for $\mathrm{C}_{21} \mathrm{H}_{24} \mathrm{O}$ (\%): C 86.26, H 8.27, Found: C 86.38, H 8.15. 
19. Synthesis of 1',1'-dimethyl-5'-phenyl-7'-tosyl-1',6',7',8'-tetrahydrospiro[cyclooctane-1,3'-furo[3,4-e]isoindole] (3mi). (zyc-2-10)

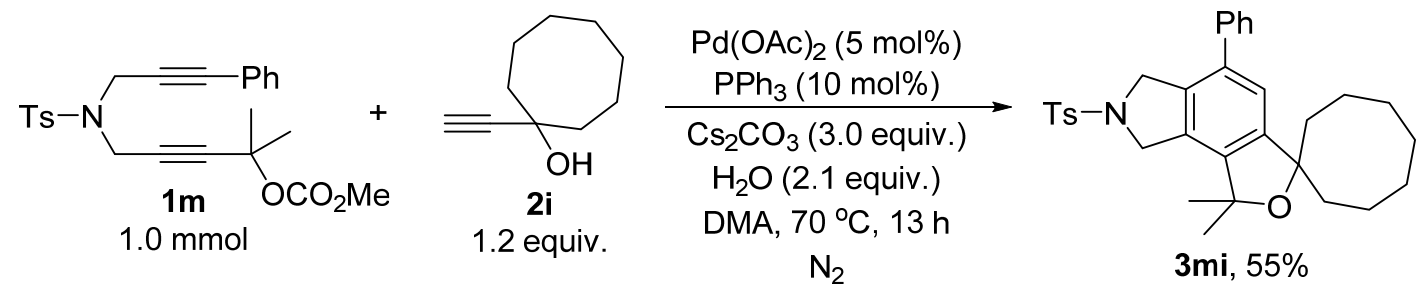

Following Typical Procedure, the reaction of $\mathrm{Cs}_{2} \mathrm{CO}_{3}(977.5 \mathrm{mg}, 3.0 \mathrm{mmol})$, $\mathrm{Pd}(\mathrm{OAc})_{2}(11.8 \mathrm{mg}, 0.05 \mathrm{mmol}), \mathrm{PPh}_{3}(26.7 \mathrm{mg}, 0.1 \mathrm{mmol}), 2 \mathbf{i}(183.3 \mathrm{mg}, 1.2 \mathrm{mmol})$, 1m (439.6 mg, $1.0 \mathrm{mmol})$, and $\mathrm{H}_{2} \mathrm{O}(37.5 \mathrm{mg}, 2.1 \mathrm{mmol})$ in DMA (10 mL) afforded 3mi $(281.6 \mathrm{mg}, 55 \%)$ (eluent: petroleum ether/ethyl acetate $=20 / 1(1000 \mathrm{~mL})$ to $10 / 1$ $(440 \mathrm{~mL}))$ as a solid; m.p. $176.5-177.3{ }^{\circ} \mathrm{C}$ (n-hexane/DCM); ${ }^{1} \mathrm{H}$ NMR $(300 \mathrm{MHz}$, $\left.\mathrm{CDCl}_{3}\right) \delta 7.75(\mathrm{~d}, J=8.1 \mathrm{~Hz}, 2 \mathrm{H}, \mathrm{ArH}), 7.50-7.34(\mathrm{~m}, 3 \mathrm{H}, \mathrm{ArH}), 7.33-7.27(\mathrm{~m}, 4 \mathrm{H}$, ArH), 7.01 (s, $1 \mathrm{H}, \mathrm{ArH}), 4.66\left(\mathrm{~s}, 2 \mathrm{H}, \mathrm{NCH}_{2}\right), 4.60\left(\mathrm{~s}, 2 \mathrm{H}, \mathrm{NCH}_{2}\right), 2.40\left(\mathrm{~s}, 3 \mathrm{H}, \mathrm{CH}_{3}\right)$, 2.10-1.92 (m, $\left.2 \mathrm{H}, \mathrm{CH}_{2}\right), 1.92-1.60\left(\mathrm{~m}, 9 \mathrm{H}, \mathrm{CH}_{2} \times 4\right.$ and one proton of $\left.\mathrm{CH}_{2}\right)$, 1.60-1.42 (m, $9 \mathrm{H}, \mathrm{CH}_{3} \times 2, \mathrm{CH}_{2}$ and one proton of $\left.\mathrm{CH}_{2}\right) ;{ }^{13} \mathrm{C} \mathrm{NMR}\left(75 \mathrm{MHz}, \mathrm{CDCl}_{3}\right)$ $\delta 148.0,143.7,139.8,139.6,136.7,134.2,133.5,129.8,129.4,128.7,128.1,127.6$, $127.5,121.5,88.9,83.2,52.9,51.4,38.5,29.0,28.2,24.9,22.3,21.5$; IR (KBr) $v$ $\left(\mathrm{cm}^{-1}\right)$ 3058, 3024, 2972, 2923, 2850, 1596, 1493, 1470, 1446, 1350, 1276, 1164, 1097, 1066; MS (EI): m/z (\%) $515\left(\mathrm{M}^{+}, 40.89\right), 444$ (100); Anal. Calcd. for $\mathrm{C}_{32} \mathrm{H}_{37} \mathrm{NO}_{3} \mathrm{~S}$ (\%): C 74.53, H 7.23, N 2.72, Found: C 74.33, H 7.33, N 2.43.

20. Synthesis of 1,1-dimethyl-5-phenyl-6-propyl-6,8-dihydro-1H-spiro[benzo[1,2-c:3, 
4-c']difuran-3,1'-cyclooctane] (3ni). (zyc-2-13)

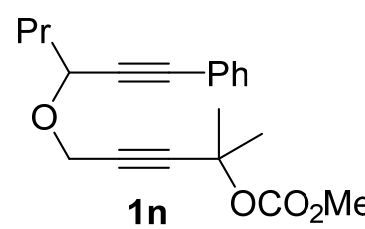

$1.0 \mathrm{mmol}$

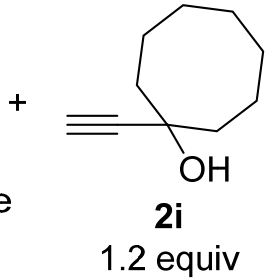

1.2 equiv

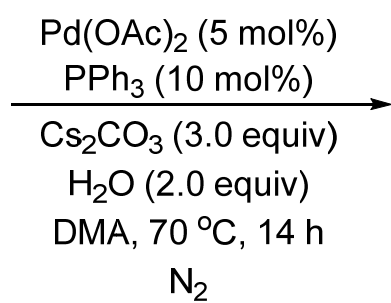

$\mathrm{N}_{2}$

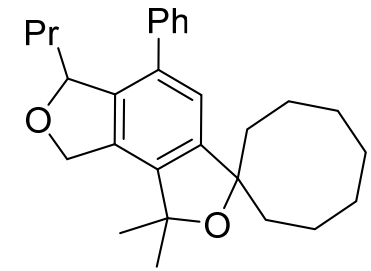

3 ni, $57 \%$

Following Typical Procedure, the reaction of $\mathrm{Cs}_{2} \mathrm{CO}_{3}(976.1 \mathrm{mg}, 3.0 \mathrm{mmol})$,

$\mathrm{Pd}(\mathrm{OAc})_{2}(11.3 \mathrm{mg}, 0.05 \mathrm{mmol}), \mathrm{PPh}_{3}(27.1 \mathrm{mg}, 0.1 \mathrm{mmol}), 2 \mathbf{i}(183.2 \mathrm{mg}, 1.2 \mathrm{mmol})$, 1n $(328.6 \mathrm{mg}, 1.0 \mathrm{mmol})$, and $\mathrm{H}_{2} \mathrm{O}(36.7 \mathrm{mg}, 2.0 \mathrm{mmol})$ in DMA $(10 \mathrm{~mL})$ afforded 3ni $(230.9 \mathrm{mg}, 57 \%)$ (eluent: petroleum ether/ethyl acetate $=40 / 1)$ as a liquid: ${ }^{1} \mathrm{H}$ NMR (300 MHz, $\left.\mathrm{CDCl}_{3}\right) \delta$ 7.48-7.29 (m, $\left.5 \mathrm{H}, \mathrm{ArH}\right), 7.00(\mathrm{~s}, 1 \mathrm{H}, \mathrm{ArH})$, 5.68-5.57 (m, $1 \mathrm{H}, \mathrm{OCH}), 5.23-5.08\left(\mathrm{~m}, 2 \mathrm{H}, \mathrm{OCH}_{2}\right), 2.23-1.62\left(\mathrm{~m}, 11 \mathrm{H}, \mathrm{CH}_{2} \times 5\right.$ and one proton of $\left.\mathrm{CH}_{2}\right), 1.62-1.41\left(\mathrm{~m}, 9 \mathrm{H}, \mathrm{CH}_{3} \times 2, \mathrm{CH}_{2}\right.$ and one proton of $\left.\mathrm{CH}_{2}\right), 1.35-1.10(\mathrm{~m}, 4 \mathrm{H}$, $\left.\mathrm{CH}_{2} \times 2\right), 0.69\left(\mathrm{t}, J=7.1 \mathrm{~Hz}, 3 \mathrm{H}, \mathrm{CH}_{3}\right) ;{ }^{13} \mathrm{C} \mathrm{NMR}\left(75 \mathrm{MHz}, \mathrm{CDCl}_{3}\right) \delta$ 147.1, 140.5, $139.7,138.5,135.8,132.4,128.4,128.2,127.2,121.5,89.1,83.3,83.2,69.8,38.6$, $38.3,36.1,29.1,28.9,28.3,28.2,24.9,22.4,22.2,18.3,13.6$; IR (neat) $v\left(\mathrm{~cm}^{-1}\right) 3080$, $3059,3028,2955,2925,2865,2852,1601,1575,1499,1471,1447,1402,1376,1359$, 1331, 1281, 1250, 1161, 1137, 1119, 1097, 1071, 1041, 1003; MS (EI): m/z (\%) 404 $\left(\mathrm{M}^{+}, 45.41\right), 333$ (100); HRMS calcd. for $\mathrm{C}_{28} \mathrm{H}_{36} \mathrm{O}_{2}\left[\mathrm{M}^{+}\right]$: 404.2715 ; Found: 404.2713.

21. Synthesis of (R)-1,1-dimethyl-5-phenyl-6-propyl-6,8-dihydro-1H-spiro[benzo[1, 2-c:3,4-c']difuran-3,1'-cyclooctane] ((R)-3ni). (zyc-2-19) 


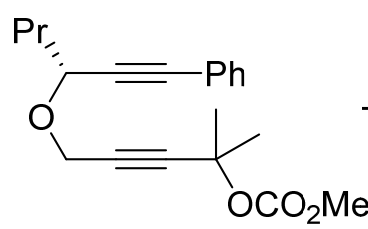

$(R)-1 \mathrm{n}$, ee $>99 \%$

$1.0 \mathrm{mmol}$

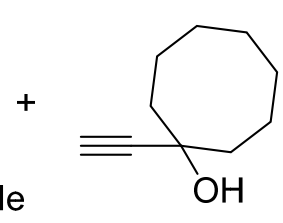

2i

1.2 equiv.

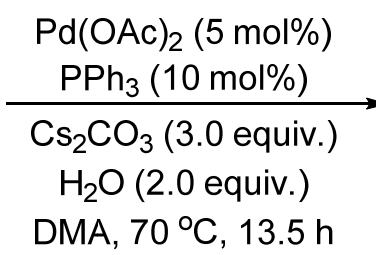

$\mathrm{N}_{2}$

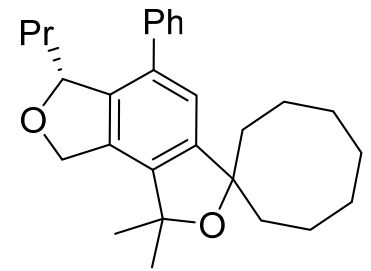

$(R)-3 n i, 57 \%$, ee $=98 \%$

Following Typical Procedure, the reaction of $\mathrm{Cs}_{2} \mathrm{CO}_{3}(975.4 \mathrm{mg}, 3.0 \mathrm{mmol})$, $\mathrm{Pd}(\mathrm{OAc})_{2}(11.4 \mathrm{mg}, 0.05 \mathrm{mmol}), \mathrm{PPh}_{3}(26.9 \mathrm{mg}, 0.1 \mathrm{mmol}), 2 \mathrm{i}(182.9 \mathrm{mg}, 1.2 \mathrm{mmol})$, (R)-1n (328.3 mg, $1.0 \mathrm{mmol},>99 \%$ ee), and $\mathrm{H}_{2} \mathrm{O}(36.5 \mathrm{mg}, 2.0 \mathrm{mmol})$ in DMA (10 $\mathrm{mL}$ ) afforded (R)-3ni (229.8 mg, 57\%) (eluent: petroleum ether/ethyl acetate/ $\mathrm{Et}_{2} \mathrm{O}=$ 100/2/1) as a liquid: $98 \%$ ee (HPLC condition: Chiralcel IA column, $n$-hexane/i-PrOH $=400 / 1,1.0 \mathrm{~mL} / \mathrm{min}, \lambda=214 \mathrm{~nm}, \mathrm{t}_{\mathrm{R}}($ major $)=6.2 \mathrm{~min}, \mathrm{t}_{\mathrm{R}}($ minor $\left.)=7.8 \mathrm{~min}\right) ;[\alpha]_{\mathrm{D}}{ }^{20}$ $=-41.8\left(\mathrm{c}=0.965, \mathrm{CHCl}_{3}\right) ;{ }^{1} \mathrm{H} \mathrm{NMR}\left(300 \mathrm{MHz}, \mathrm{CDCl}_{3}\right) \delta 7.50-7.29(\mathrm{~m}, 5 \mathrm{H}, \mathrm{ArH})$, $7.00(\mathrm{~s}, 1 \mathrm{H}, \mathrm{ArH}), 5.68-5.57(\mathrm{~m}, 1 \mathrm{H}, \mathrm{OCH}), 5.23-5.08\left(\mathrm{~m}, 2 \mathrm{H}, \mathrm{OCH}_{2}\right), 2.23-1.62(\mathrm{~m}$, $11 \mathrm{H}, \mathrm{CH}_{2} \times 5$ and one proton of $\left.\mathrm{CH}_{2}\right), 1.62-1.41\left(\mathrm{~m}, 9 \mathrm{H}, \mathrm{CH}_{3} \times 2, \mathrm{CH}_{2}\right.$ and one proton of $\left.\mathrm{CH}_{2}\right), 1.35-1.10\left(\mathrm{~m}, 4 \mathrm{H}, \mathrm{CH}_{2} \times 2\right), 0.69\left(\mathrm{t}, J=7.1 \mathrm{~Hz}, 3 \mathrm{H}, \mathrm{CH}_{3}\right) ;{ }^{13} \mathrm{C} \mathrm{NMR}$ $\left(75 \mathrm{MHz}, \mathrm{CDCl}_{3}\right) \delta 147.2,140.5,139.7,138.5,135.9,132.4,128.4,128.2,127.2$ $121.6,89.2,83.4,83.3,69.9,38.7,38.3,36.1,29.1,28.9,28.4,28.2,25.0,22.4,22.3$, 18.3, 13.7; IR (neat) $v\left(\mathrm{~cm}^{-1}\right)$ 3062, 3028, 2959, 2925, 2869, 2852, 1601, 1570, 1500 , 1472, 1447, 1376, 1359, 1331, 1281, 1161, 1137, 1071, 1041, 1008; MS (EI): m/z (\%) $404\left(\mathrm{M}^{+}\right.$, 53.55), 333 (100); HRMS calcd. for $\mathrm{C}_{28} \mathrm{H}_{36} \mathrm{O}_{2}\left[\mathrm{M}^{+}\right]$: 404.2715; Found: 404.2717 .

22. Synthesis of 8-hexyl-1,1,3,3-tetramethyl-5-phenyl-1,3,6,8-tetrahydrobenzo[1,2-c: 3,4-c']difuran (3oa). (zyc-2-14) 


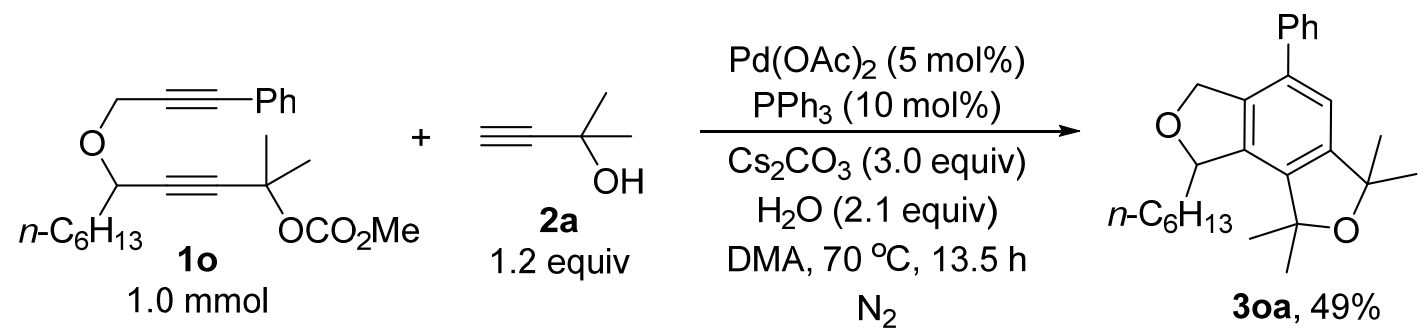

Following Typical Procedure, the reaction of $\mathrm{Cs}_{2} \mathrm{CO}_{3}(976.2 \mathrm{mg}, 3.0 \mathrm{mmol})$, $\mathrm{Pd}(\mathrm{OAc})_{2}(11.3 \mathrm{mg}, 0.05 \mathrm{mmol}), \mathrm{PPh}_{3}(26.9 \mathrm{mg}, 0.1 \mathrm{mmol}), 2 \mathrm{a}(103.9 \mathrm{mg}, 1.2 \mathrm{mmol})$, 10 (370.0 mg, $1.0 \mathrm{mmol})$, and $\mathrm{H}_{2} \mathrm{O}(37.1 \mathrm{mg}, 2.1 \mathrm{mmol})$ in DMA (10 mL) afforded 3oa $(185.2 \mathrm{mg}, 49 \%)$ (eluent: petroleum ether/ethyl acetate $=50 / 1)$ as a liquid: ${ }^{1} \mathrm{H}$ NMR (300 MHz, $\left.\mathrm{CDCl}_{3}\right) \delta$ 7.48-7.27 (m, $\left.5 \mathrm{H}, \mathrm{ArH}\right), 7.03(\mathrm{~s}, 1 \mathrm{H}, \mathrm{ArH})$, 5.47-5.36 (m, $1 \mathrm{H}, \mathrm{OCH}), 5.28\left(\mathrm{dd}, J_{1}=12.6 \mathrm{~Hz}, J_{2}=2.4 \mathrm{~Hz}, 1 \mathrm{H}\right.$, one proton of $\left.\mathrm{OCH}_{2}\right), 4.93(\mathrm{~d}, J$ $=12.6 \mathrm{~Hz}, 1 \mathrm{H}$, one proton of $\left.\mathrm{OCH}_{2}\right), 1.97-1.78\left(\mathrm{~m}, 2 \mathrm{H}, \mathrm{CH}_{2}\right), 1.66\left(\mathrm{~s}, 3 \mathrm{H}, \mathrm{CH}_{3}\right)$, 1.62-1.47 (m, $11 \mathrm{H}, \mathrm{CH}_{3} \times 3$ and $\left.\mathrm{CH}_{2}\right), 1.47-1.23\left(\mathrm{~m}, 6 \mathrm{H}, \mathrm{CH}_{2} \times 3\right), 0.89(\mathrm{t}, J=6.8$ $\left.\mathrm{Hz}, 3 \mathrm{H}, \mathrm{CH}_{3}\right) ;{ }^{13} \mathrm{C} \mathrm{NMR}\left(75 \mathrm{MHz}, \mathrm{CDCl}_{3}\right) \delta 147.5,140.0,138.2,137.8,136.2,135.7$, $128.5,127.8,127.3,120.4,84.0,83.7,82.6,71.1,35.6,31.7,31.0,30.94,30.88,29.1$, 28.8, 25.6, 22.5, 14.0; IR (neat) $v\left(\mathrm{~cm}^{-1}\right) 3058,3028,2969,2926,2856,1602,1570$, $1501,1467,1446,1373,1361,1339,1272,1246,1175,1161,1139,1106,1052 ; \mathrm{MS}$ (EI): $m / z(\%) 378\left(\mathrm{M}^{+}, 5.20\right), 363$ (100), 293 (100); HRMS calcd. for $\mathrm{C}_{26} \mathrm{H}_{34} \mathrm{O}_{2}\left[\mathrm{M}^{+}\right]$: 378.2559; Found: 378.2562.

23. Synthesis of (R)-8-hexyl-1,1,3,3-tetramethyl-5-phenyl-1,3,6,8-tetrahydrobenzo[1, 2-c:3,4-c']difuran ((R)-3oa). (zyc-2-20) 


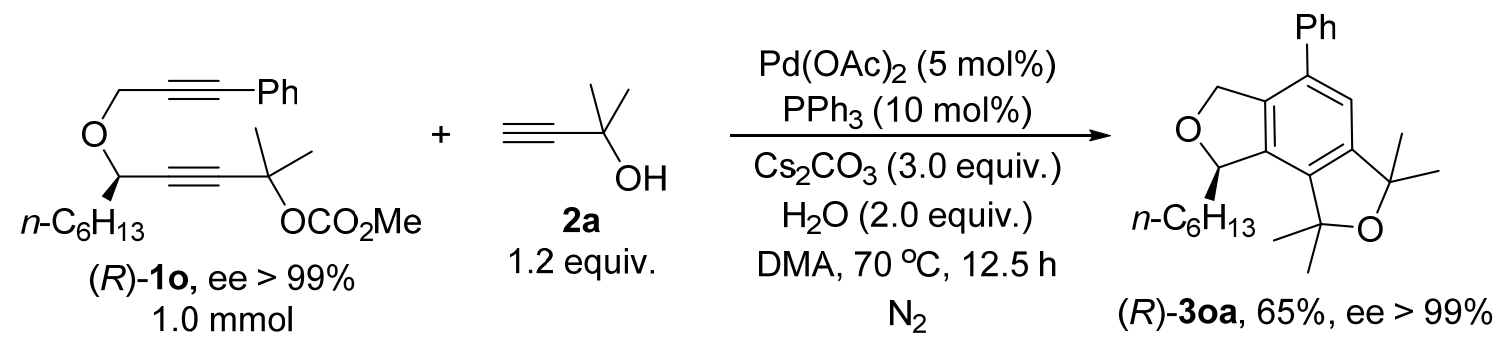

Following Typical Procedure, the reaction of $\mathrm{Cs}_{2} \mathrm{CO}_{3}(976.0 \mathrm{mg}, 3.0 \mathrm{mmol})$, $\mathrm{Pd}(\mathrm{OAc})_{2}(11.5 \mathrm{mg}, 0.05 \mathrm{mmol}), \mathrm{PPh}_{3}(26.6 \mathrm{mg}, 0.1 \mathrm{mmol}), 2 \mathrm{a}(103.5 \mathrm{mg}, 1.2 \mathrm{mmol})$, (R)-10 (370.9 mg, $1.0 \mathrm{mmol})$, and $\mathrm{H}_{2} \mathrm{O}(36.2 \mathrm{mg}, 2.0 \mathrm{mmol})$ in DMA (10 mL) afforded $(R)$-3oa $(246.5 \mathrm{mg}, 65 \%)$ (eluent: petroleum ether/ethyl acetate/DCM = 50/1/1) as a liquid: $>99 \%$ ee (HPLC condition: Chiralcel IA column, $n$-hexane $/ \mathrm{i}-\mathrm{PrOH}=400 / 1,1.0 \mathrm{~mL} / \mathrm{min}, \lambda=214 \mathrm{~nm}, \mathrm{t}_{\mathrm{R}}($ major $)=7.3 \mathrm{~min} ;[\alpha]_{\mathrm{D}}^{20}=$ $+26.2\left(\mathrm{c}=0.960, \mathrm{CHCl}_{3}\right) ;{ }^{1} \mathrm{H} \mathrm{NMR}\left(300 \mathrm{MHz}, \mathrm{CDCl}_{3}\right) \delta$ 7.48-7.27 (m, $\left.5 \mathrm{H}, \mathrm{ArH}\right)$, $7.03(\mathrm{~s}, 1 \mathrm{H}, \mathrm{ArH}), 5.47-5.36(\mathrm{~m}, 1 \mathrm{H}, \mathrm{OCH}), 5.27\left(\mathrm{dd}, J_{1}=12.6 \mathrm{~Hz}, J_{2}=2.4 \mathrm{~Hz}, 1 \mathrm{H}\right.$, one proton of $\left.\mathrm{OCH}_{2}\right), 4.93\left(\mathrm{~d}, J=12.6 \mathrm{~Hz}, 1 \mathrm{H}\right.$, one proton of $\left.\mathrm{OCH}_{2}\right), 1.97-1.78(\mathrm{~m}, 2$ $\left.\mathrm{H}, \mathrm{CH}_{2}\right), 1.66\left(\mathrm{~s}, 3 \mathrm{H}, \mathrm{CH}_{3}\right), 1.62-1.47\left(\mathrm{~m}, 11 \mathrm{H}, \mathrm{CH}_{3} \times 3\right.$ and $\left.\mathrm{CH}_{2}\right), 1.47-1.23(\mathrm{~m}, 6 \mathrm{H}$, $\left.\mathrm{CH}_{2} \times 3\right), 0.89\left(\mathrm{t}, J=6.8 \mathrm{~Hz}, 3 \mathrm{H}, \mathrm{CH}_{3}\right) ;{ }^{13} \mathrm{C} \mathrm{NMR}\left(75 \mathrm{MHz}, \mathrm{CDCl}_{3}\right) \delta 147.6,140.0$, $138.2,137.8,136.2,135.7,128.5,127.8,127.3,120.4,84.0,83.6,82.6,71.1,35.6$, $31.7,31.0,30.93,30.87,29.1,28.8,25.6,22.5,14.0$; IR (neat) $v\left(\mathrm{~cm}^{-1}\right) 3058,3028$ 2969, 2926, 2856, 1601, 1573, 1500, 1466, 1446, 1373, 1361, 1340, 1272, 1245, 1175 , 1161, 1138, 1106, 1052; MS (EI): m/z (\%) $378\left(\mathrm{M}^{+}, 4.65\right), 363$ (100), 293 (100); HRMS calcd. for $\mathrm{C}_{26} \mathrm{H}_{34} \mathrm{O}_{2}\left[\mathrm{M}^{+}\right]$: 378.2559; Found: 378.2555 .

24. Synthesis of 1,4-bis(6,6,8,8-tetramethyl-1,3-dihydrobenzo[1,2-c:3,4-c']difuran-4-yl)benzene (3pa). (zyc-2-37) 

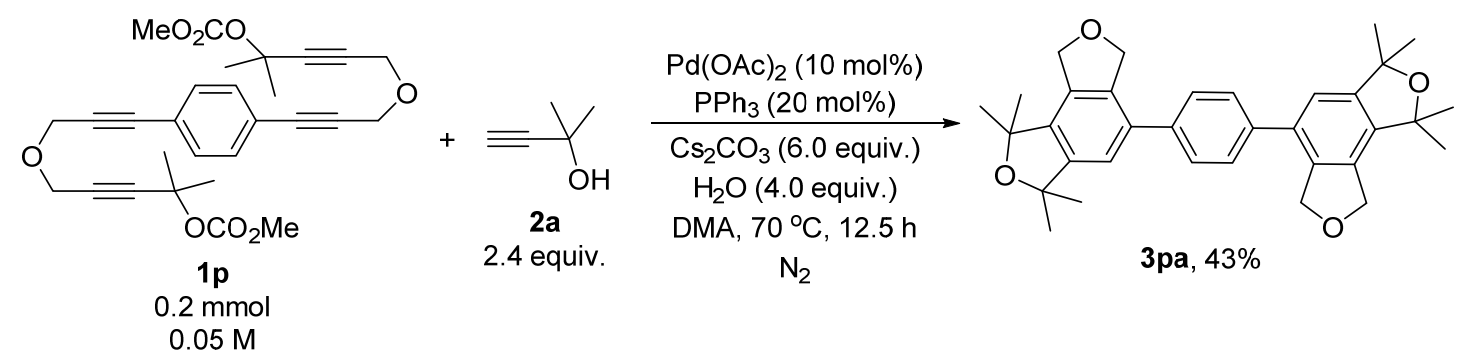

Following Typical Procedure I, the reaction of $\mathrm{Cs}_{2} \mathrm{CO}_{3}(390.1 \mathrm{mg}, 1.2 \mathrm{mmol})$, $\mathrm{Pd}(\mathrm{OAc})_{2}(4.9 \mathrm{mg}, 0.02 \mathrm{mmol}), \mathrm{PPh}_{3}(10.7 \mathrm{mg}, 0.04 \mathrm{mmol}), 2 \mathrm{a}(41.2 \mathrm{mg}, 0.48 \mathrm{mmol})$, 1p (102.0 mg, $0.2 \mathrm{mmol})$, and $\mathrm{H}_{2} \mathrm{O}(14.3 \mathrm{mg}, 0.8 \mathrm{mmol})$ in DMA (4 mL) afforded 3pa (45.2 mg, 43\%) (eluent: petroleum ether/ethyl acetate $=10 / 1)$ as a solid: m.p. > $280{ }^{\circ} \mathrm{C}$ (n-hexane/DCM); ${ }^{1} \mathrm{H}$ NMR $\left(300 \mathrm{MHz}, \mathrm{CDCl}_{3}\right) \delta 7.49$ (s, $\left.4 \mathrm{H}, \mathrm{ArH}\right), 7.10$ (s, 2 $\mathrm{H}, \mathrm{ArH}), 5.23\left(\mathrm{~s}, 4 \mathrm{H}, \mathrm{OCH}_{2} \times 2\right), 5.22\left(\mathrm{~s}, 4 \mathrm{H}, \mathrm{OCH}_{2} \times 2\right), 1.59\left(\mathrm{~s}, 12 \mathrm{H}, \mathrm{CH}_{3} \times 4\right)$, $1.56\left(\mathrm{~s}, 12 \mathrm{H}, \mathrm{CH}_{3} \times 4\right) ;{ }^{13} \mathrm{C} \mathrm{NMR}\left(75 \mathrm{MHz}, \mathrm{CDCl}_{3}\right) \delta 147.2,139.3,138.6,137.3$, 135.1, 132.5, 128.2, 120.4, 84.6, 83.7, 73.1, 71.2, 31.0, 28.9; IR (KBr) v (cm $\left.{ }^{-1}\right) 2969$, 2923, 2843, 1472, 1454, 1448, 1386, 1373, 1361, 1334, 1285, 1244, 1173, 1140, 1107 , 1061; MS (EI): m/z (\%) $510\left(\mathrm{M}^{+}, 6.69\right), 495$ (100); Anal. Calcd. for $\mathrm{C}_{34} \mathrm{H}_{38} \mathrm{O}_{4}$ (\%): C 79.97, H 7.50, Found: C 79.96, H 7.45. 


\section{References}

[1] X. Huang, W. Wu, C. Fu, Y. Yu, S. Ma, Chem. Eur. J. 2015, 21, 15540.

[2] S. Song, C. Fu, X. Huang, S. Ma, Adv. Synth. Catal. 2018, 360, 1019.

[3] Y. Zhang, W. Wu, C. Fu, X. Huang, S. Ma, Chem. Sci. 2019, doi: 10.1039/C8SC04681F 
$682^{\prime} 92$

BOS $0 E$

$9 t 1 \cdot \varepsilon t$

$8 \angle Z^{\circ} \vdash G$
$0 \triangleright 9^{\circ} 99$

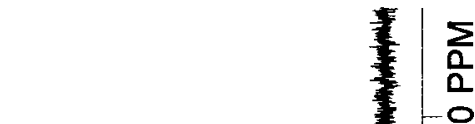

$601 \angle S$

$\angle L S^{\circ} 9 L$

$19 \angle 9 L$

$000 \angle L$

$\varepsilon Z \nabla^{\circ} L L$

$99 \varepsilon 18$

$091 \div 8$

$001 \cdot 98$

$6 \angle 9^{\circ} 98$

$\varepsilon \nabla \varepsilon 乙 乙 \downarrow$

의 92 L

$6818 Z 1$

60ย'8Zレ

LEヤ'8ZL

Z89' $L$ L

OEO'เレ

GLE'ESL

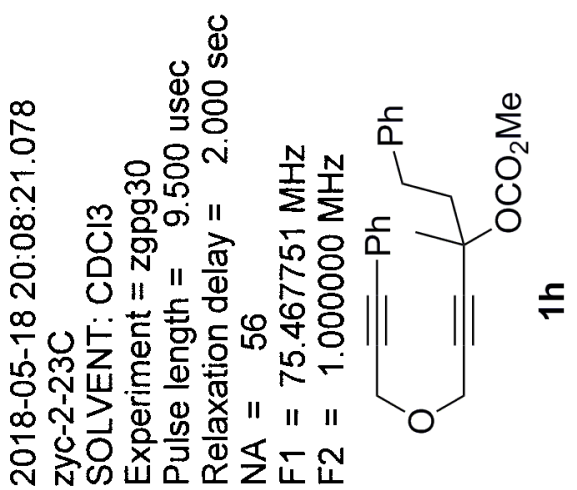



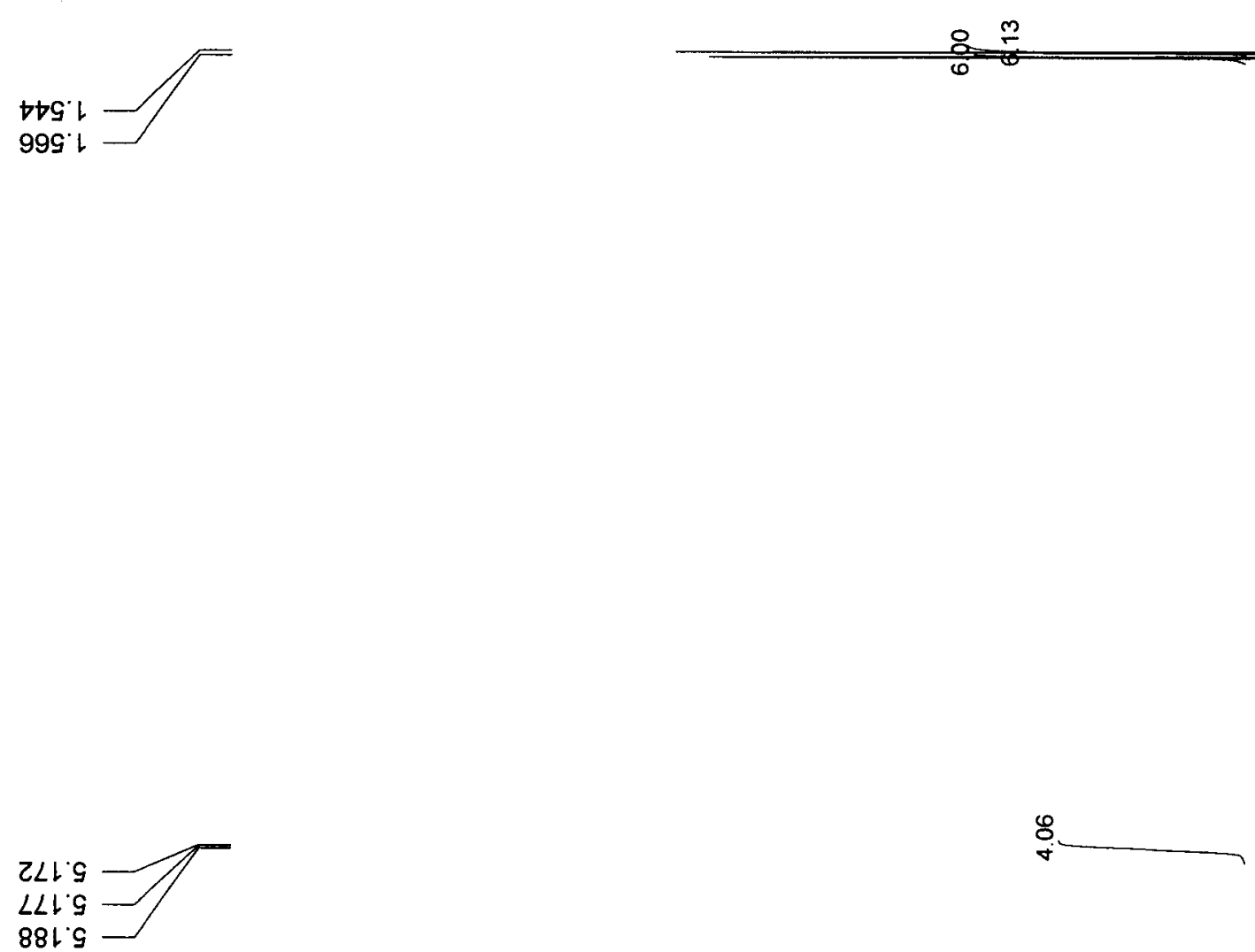

8819
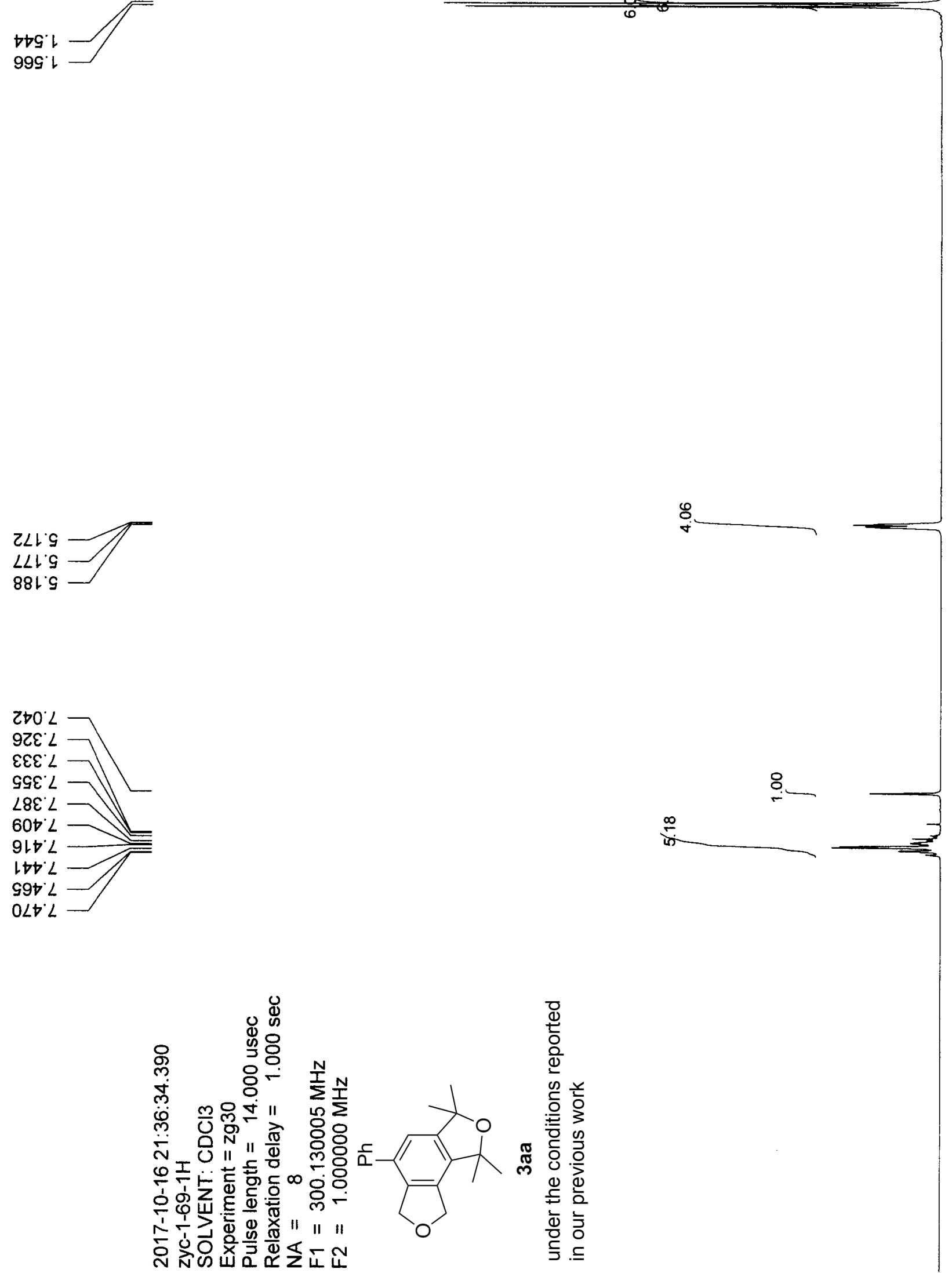

$-N$ 


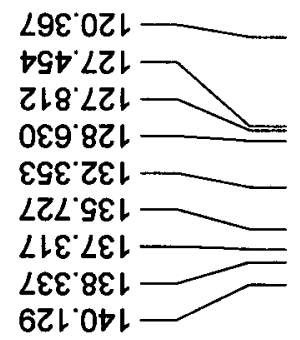

$190^{\circ} \angle t$

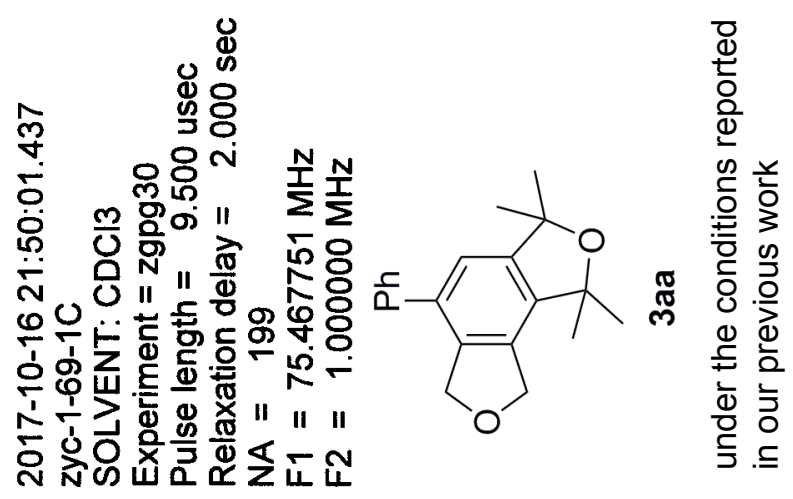


ڤ̊ํํ

690.9

$\angle O Z ' 9$ †ाZ 9

ozZ 9

$\angle 9 Z 9$

ZLZ'

$\angle L Z G$

$\angle Z E$ '

6๖E' 2

ZOt $L$

จเ十 L

$\varepsilon \varepsilon t^{\circ} L$

$\angle E \nabla L$

$190^{\circ} \mathrm{L}$

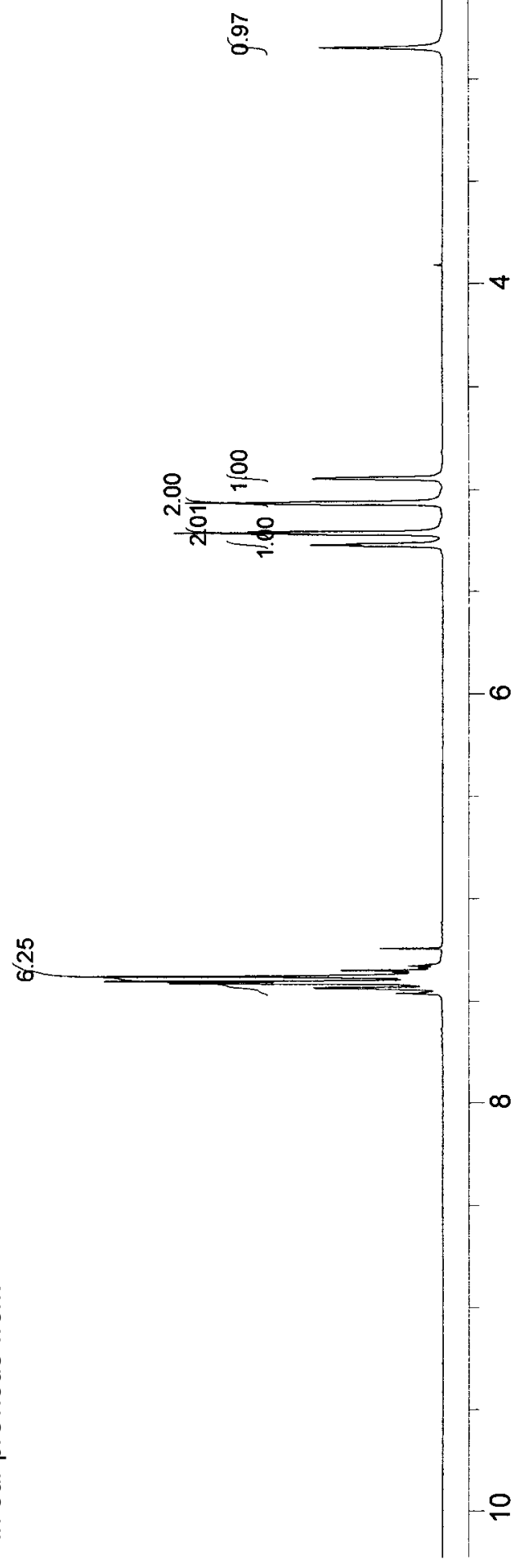


ZZ8'

$96 Z^{\circ} \varepsilon L$

$998^{\circ} \varepsilon$

$9 L L \circ L$

$\angle \angle 9^{\circ} 9$

$000 \angle L$

$\varepsilon Z \nabla^{\circ} \angle L$

OOLGLL_-

891921

GE† LZL

$8 \rightarrow L \angle Z L$

$899^{\circ} 82$

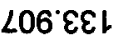

6เ乙`॰

L৮LSEL

$800^{\circ} 6 \varepsilon L$

6LOODL

$\angle \succ 8^{\circ} \mathrm{G}$ L

$\varepsilon 9 \varepsilon^{\circ} \angle \downarrow L$

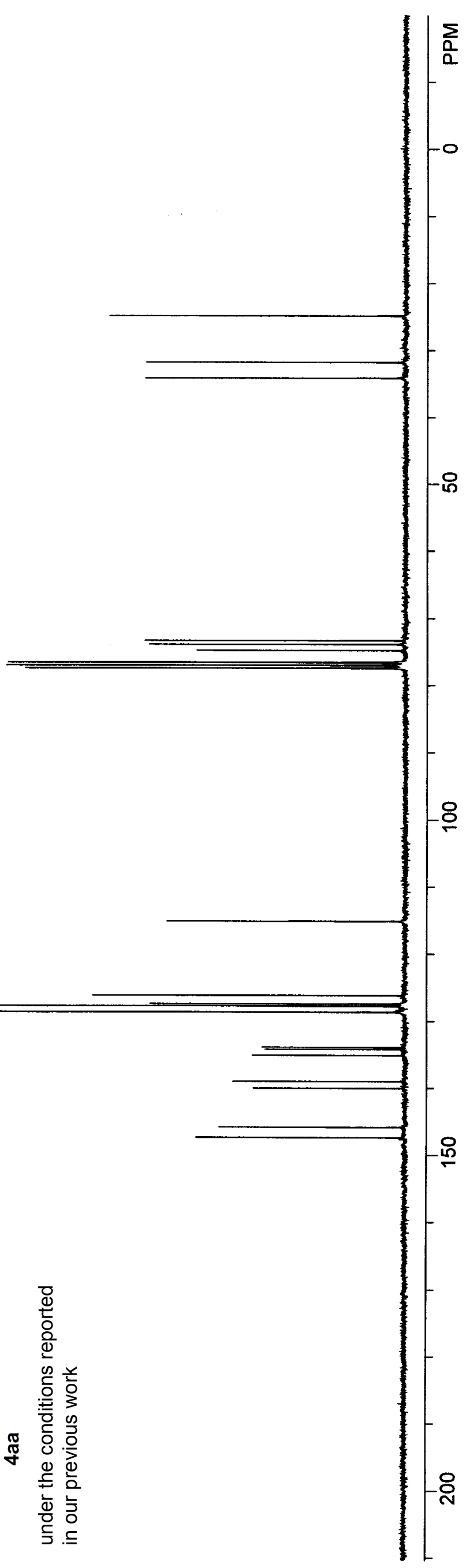




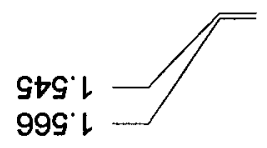

999'

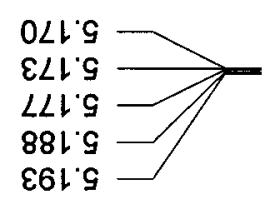

$\stackrel{2}{9}$

$\mathrm{ZtO} 0^{\circ}$

$\varepsilon \varepsilon \varepsilon$

GSE $L$

$\varepsilon 8 \varepsilon^{\circ} L$

$\angle 8 \varepsilon^{\circ} L$

$60 t^{\circ} \mathrm{L}$

$\angle L D^{\circ} \angle$

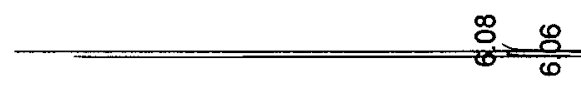

$\omega$

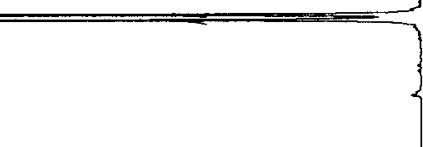

$8 \varepsilon \nabla^{\circ} L$

IVt $L$

$99 t^{\circ} \mathrm{L}$

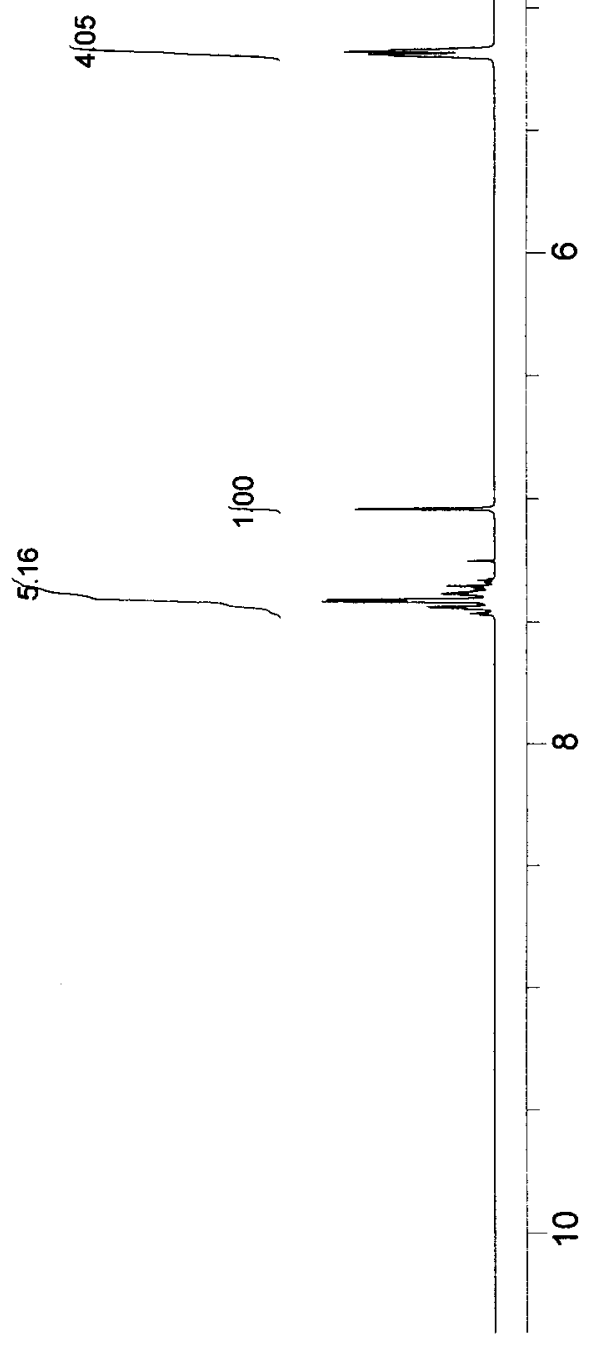




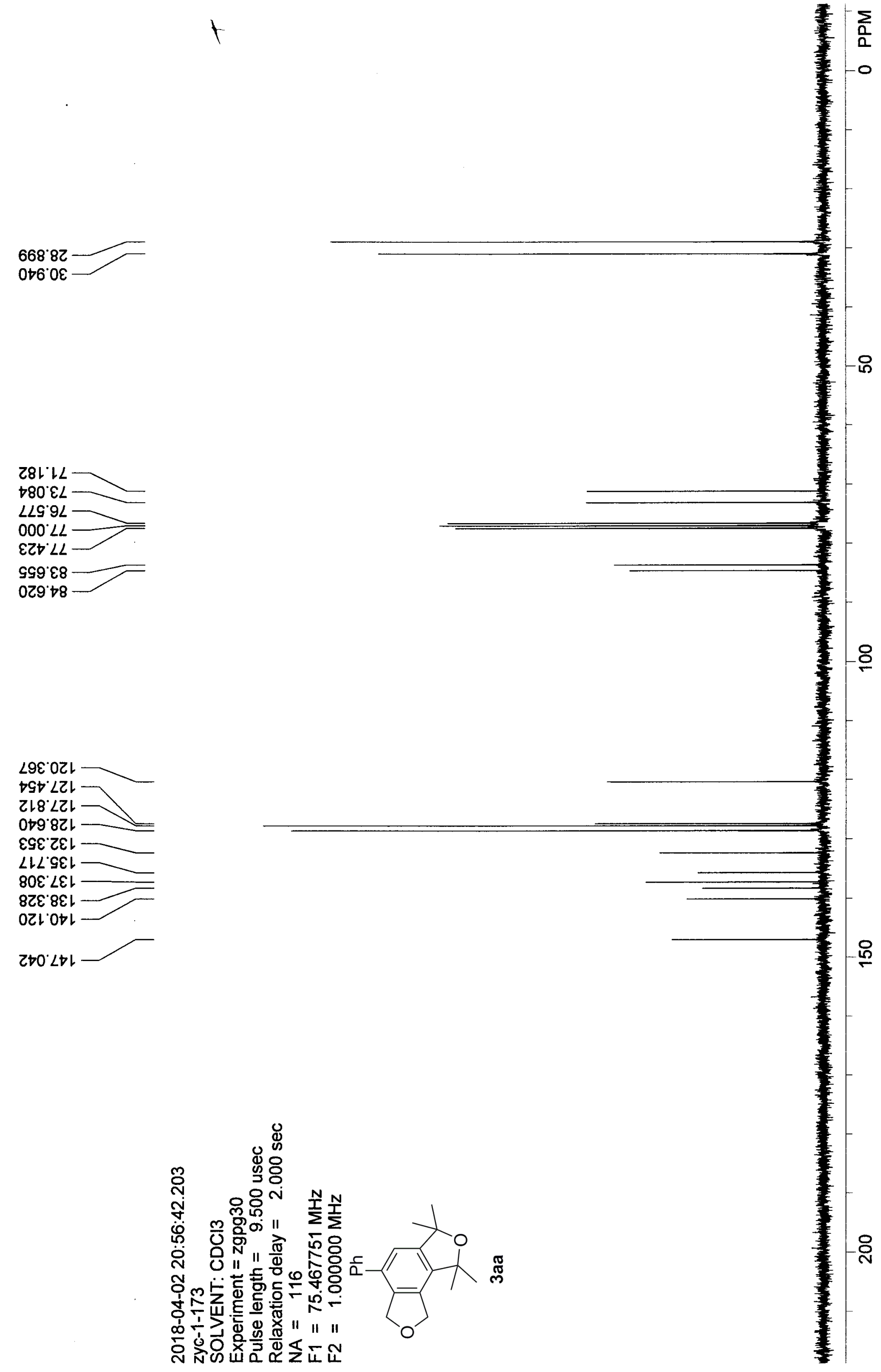



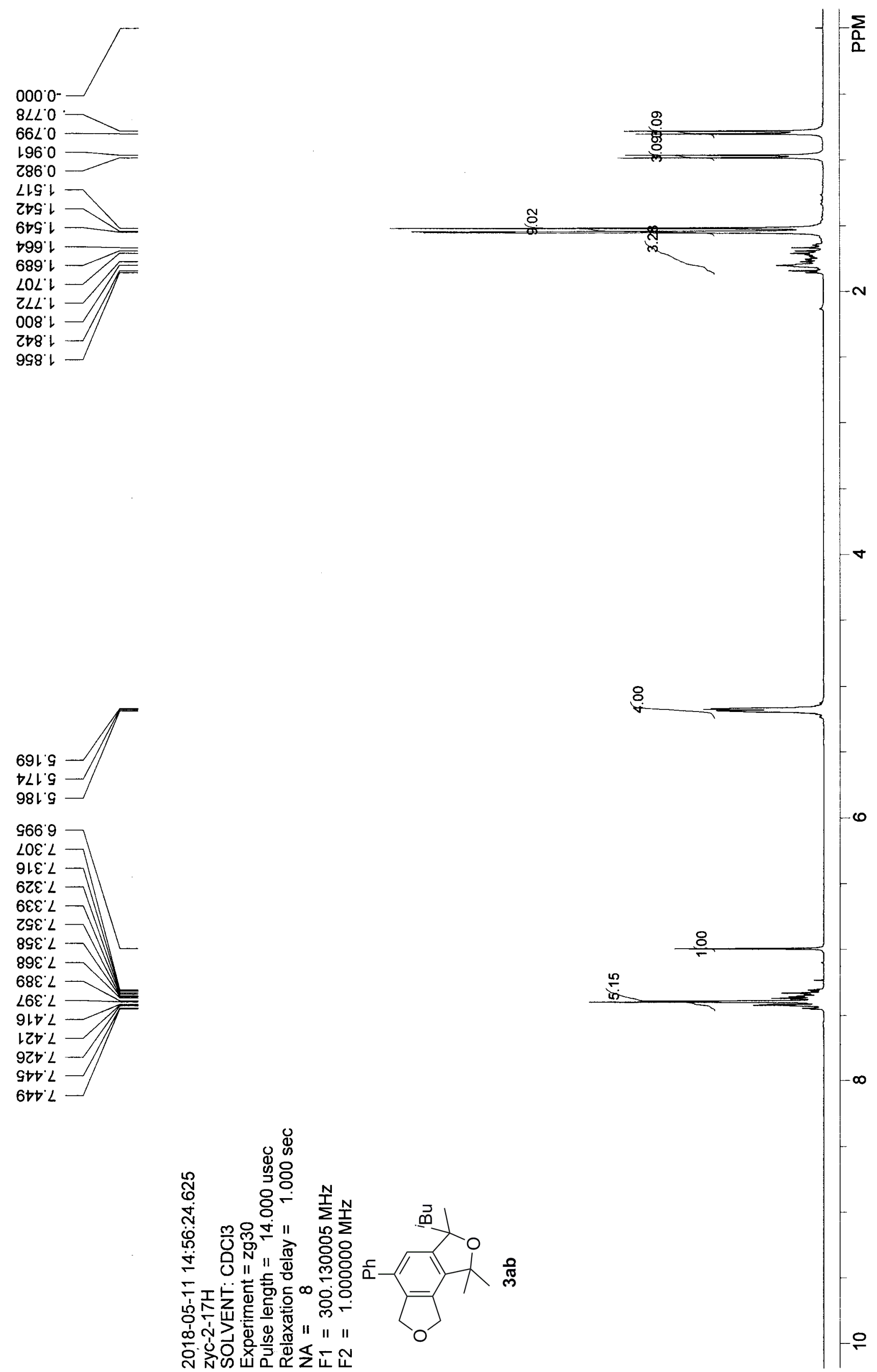
$G \angle L \circ Z$

$98 \varepsilon \triangleright 乙$

1 1092

$\angle 1082$

$0 \varepsilon Z^{\prime} 6 Z$

$297^{\circ} 0 \varepsilon$

706.09

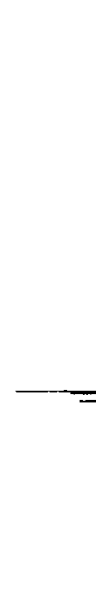

$801 \% L$

$10^{\circ} \varepsilon L$

$\angle L G$ ' $L$

$000 . \angle L$

EZt $\angle L$

$69 \mathcal{E}^{\prime} \angle 8$

$889^{\circ} 02$

SZE $\angle 2$

8Es' $8 Z 1$

$\nabla Z Z ' Z \varepsilon$

乙† $\subseteq \varepsilon l$

$0 \angle L \angle E L$

9LG $8 \varepsilon L$

621001

sts. 901

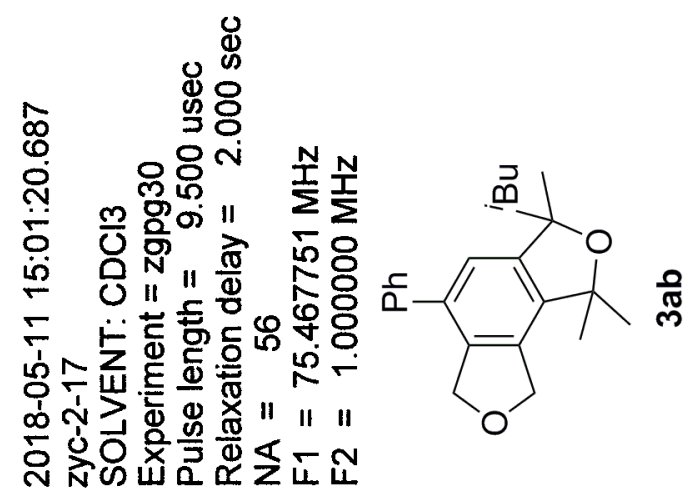

$-8$ 

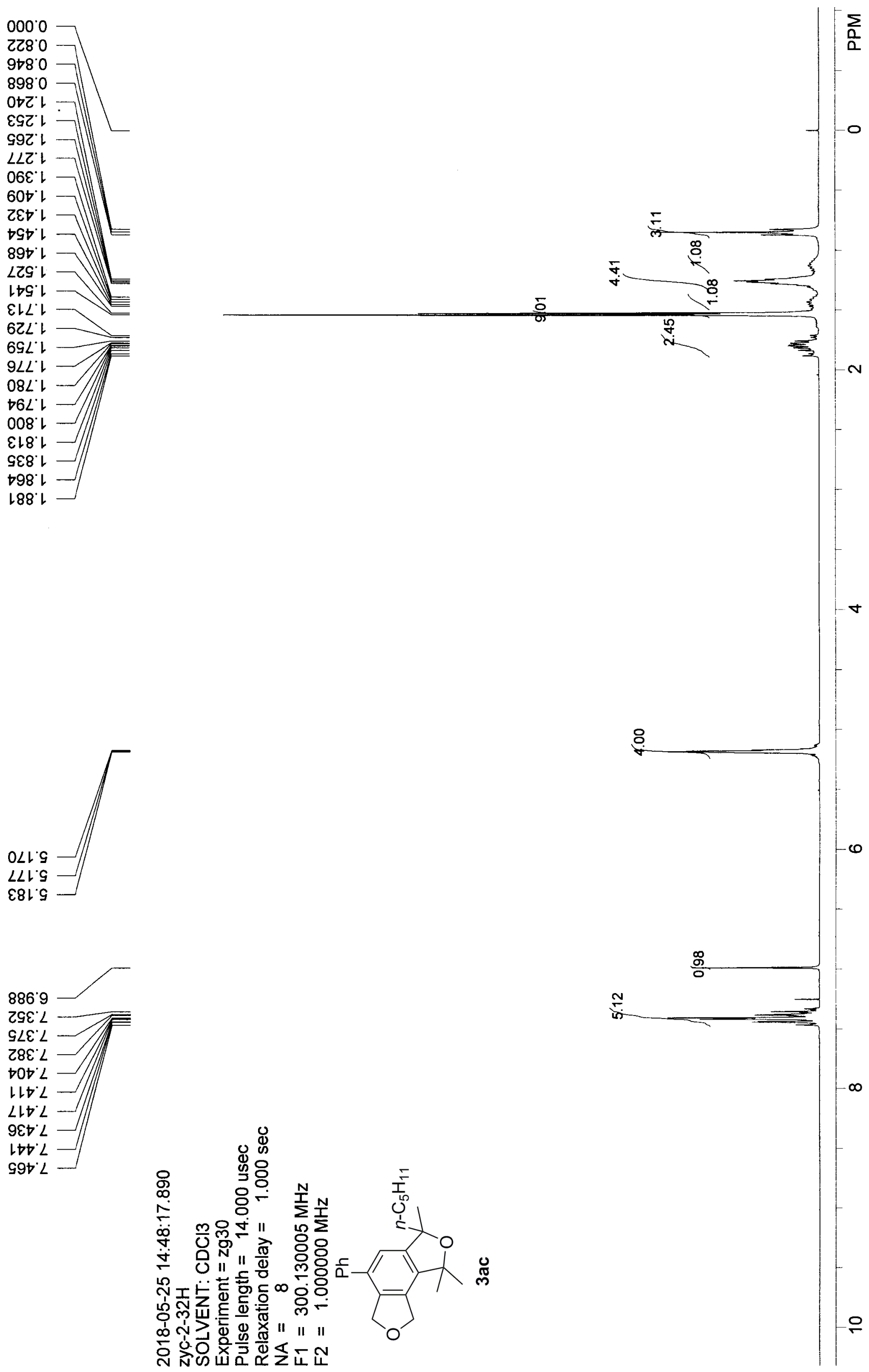
เ86'ع -

99t乙2

$\forall 90 \circ 乙$

$\angle Z L^{\circ} 8 Z$

०ะट 62

$200^{\circ} 0 \varepsilon$

$0 \angle 0 ₹ \varepsilon$

6とヤてち

Z8L'L

$\varepsilon 60^{\circ} \varepsilon$

$\angle L G^{\prime} 9 L$

$000 \angle L$

$\varepsilon 乙 \nabla^{\circ} L L$

9Z9' $\& 8$

०६ट $\angle 8$

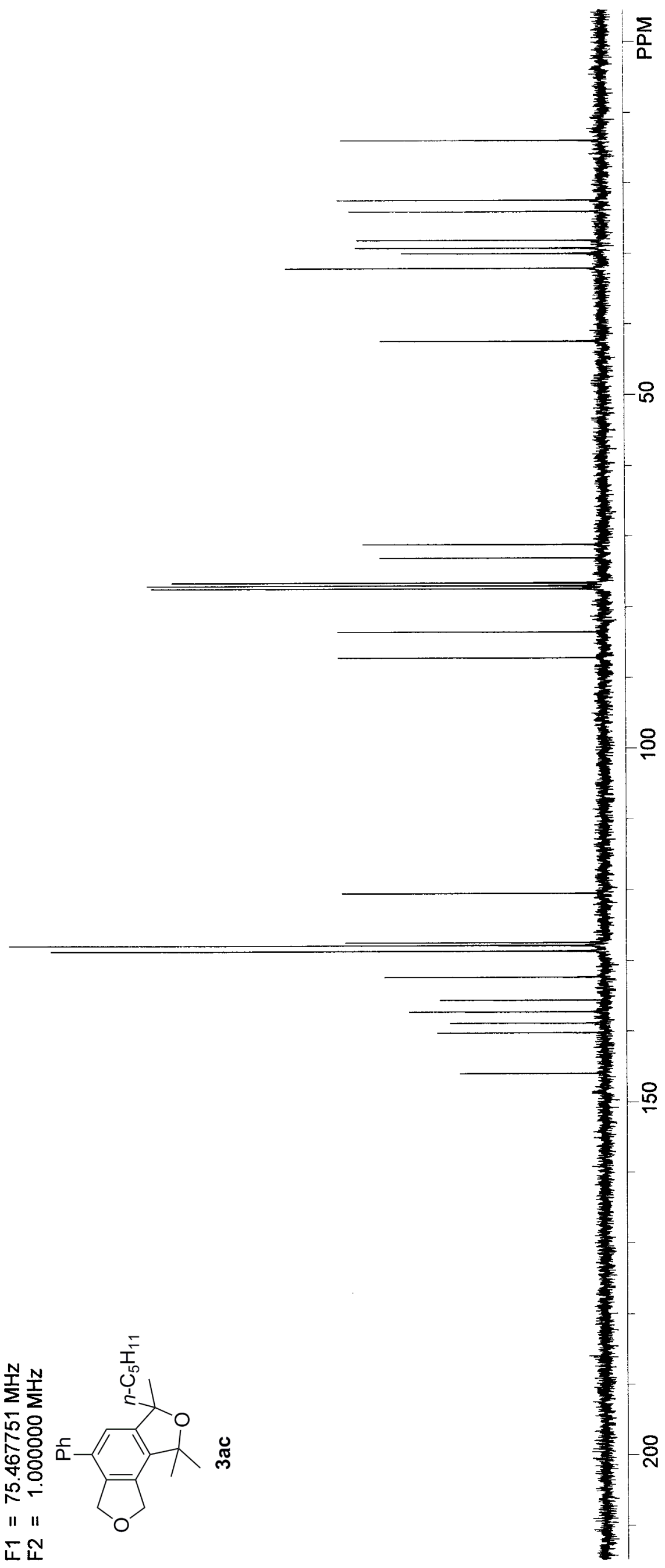



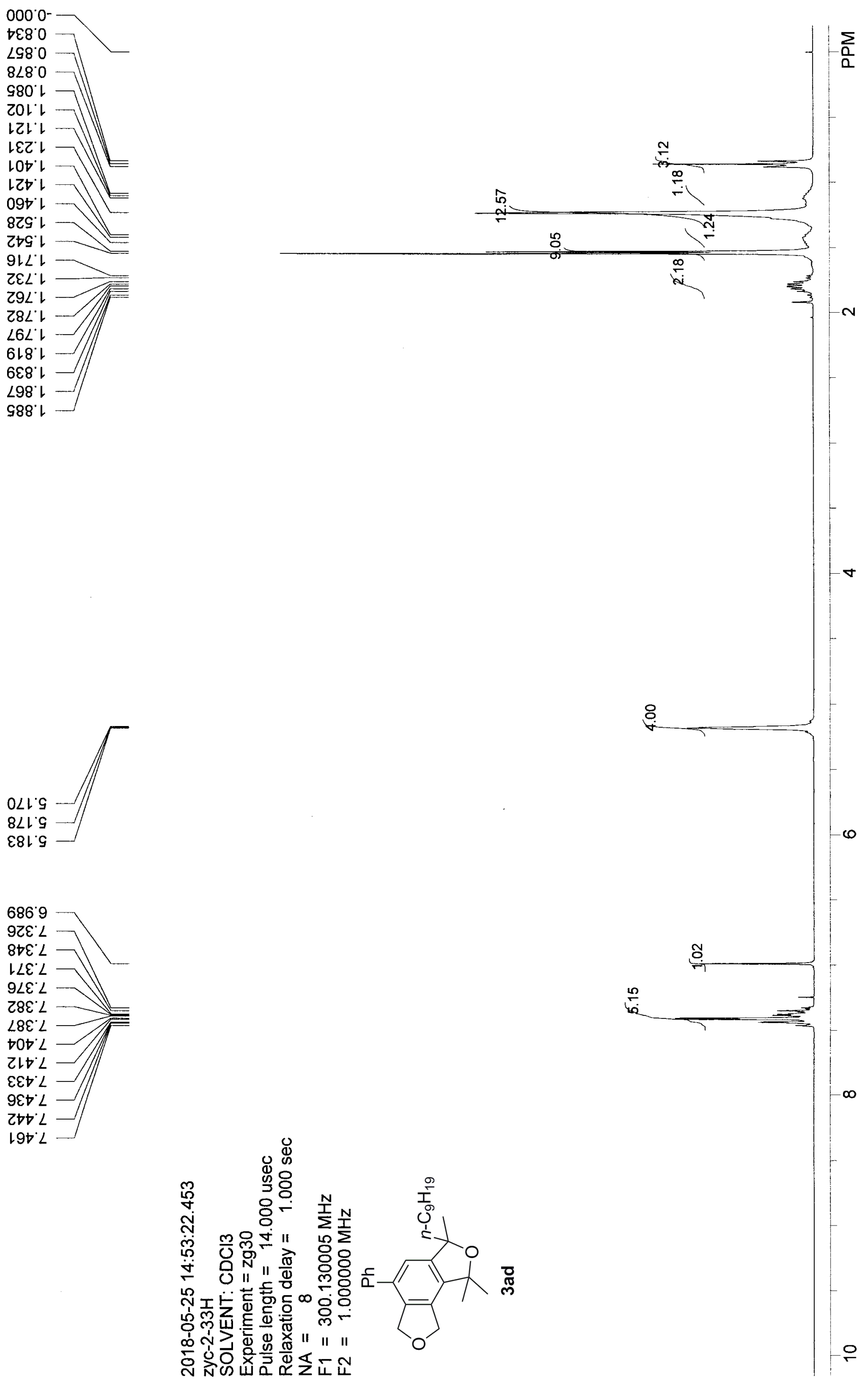
$9 \varepsilon 0^{\circ} \downarrow$

$\checkmark 69^{\prime} 22$

$\angle L \varepsilon \triangleright \tau$

LZI 82

$6 \varepsilon 262$

乙๕ 62

L87'6Z

$+98^{\circ} 62$

เ 1008

†08 $L \varepsilon$

$99 t$ Zt

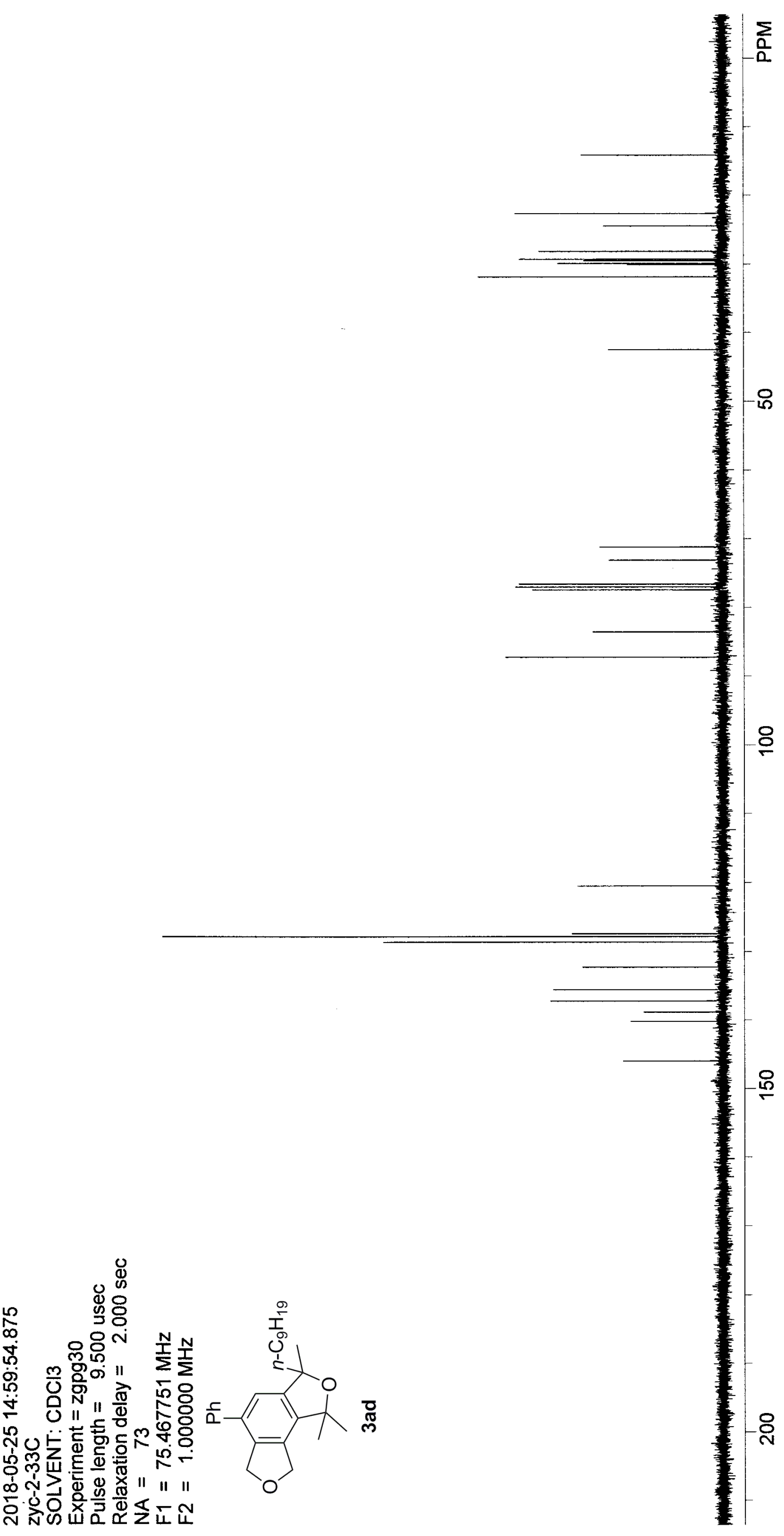

Z8L L

$\varepsilon 60^{\circ} \varepsilon L$

$\angle \angle G^{\prime} 9 \angle$

$000 \angle L$

हट๐ $L L$

9ZS $\varepsilon 8$

OtZ: $\angle 8$

$\angle \angle D O Z$

$80 T^{\circ} \angle Z$

乙Z8 $\angle Z$

Z19.8ZL

$08 Z^{\circ} Z \varepsilon$

6LG'GE

GZ乙 $\angle \varepsilon \downarrow$

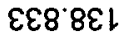

†6เ Ot

S86'Gヤ 


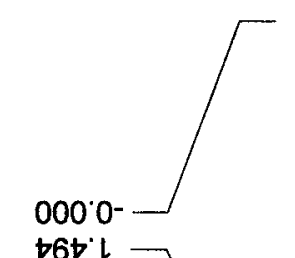

$\left\{\begin{array}{l}\sum_{0} \\ 8\end{array}\right.$

$76 t^{\circ}$

$\nabla 16$.

St6.

E96 ᄂ

$\angle L 6$.

$966^{\circ} \mathrm{L}$

2002

s.tZ

$0+t 2$

89t 2

$\nabla \angle t$

LOS' 2

1092

$\downarrow \varepsilon 9 \cdot z$

$\varepsilon \mapsto 9 \tau$

$\angle 99^{\circ} \mathrm{Z}$

$\angle \angle 9^{\circ} \mathrm{Z}$

$10 L 2$

OLLZ

$0 \angle L 9$

8

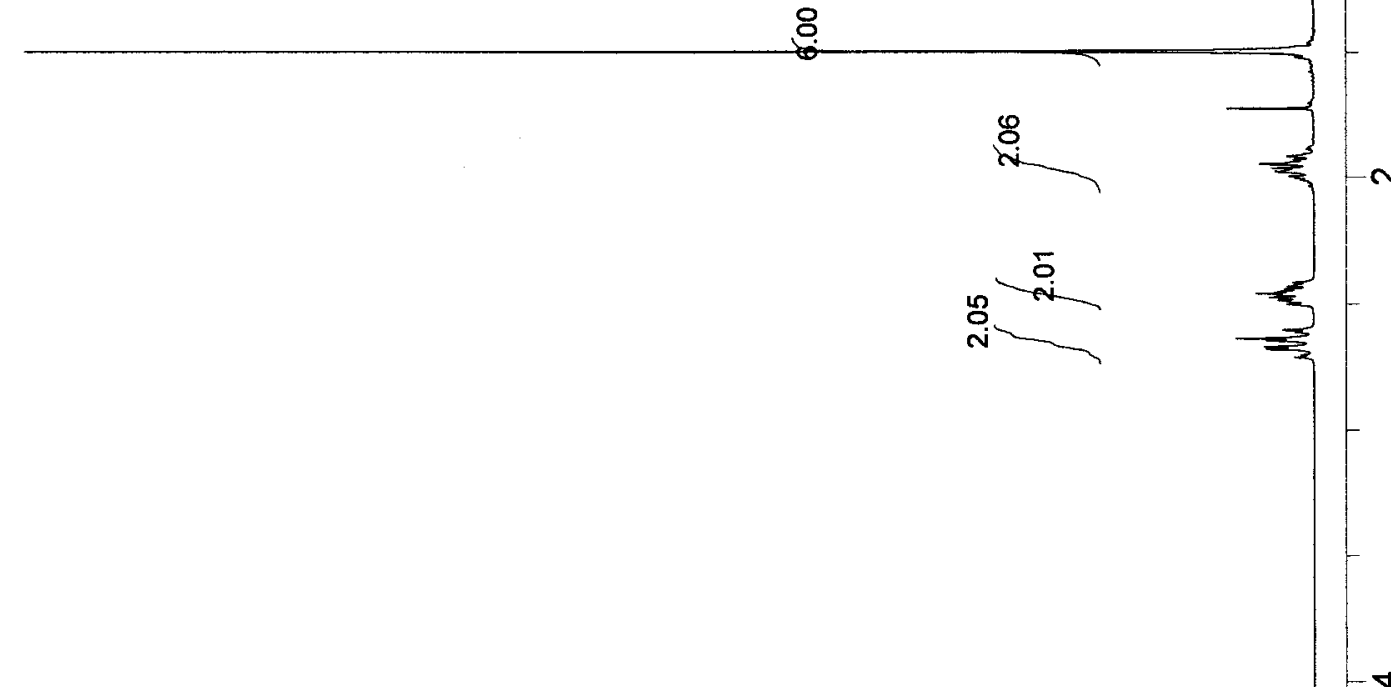

SGE $L$

$08 \varepsilon^{\prime} L$

Got' $L$

$\angle L t L$

$8 \varepsilon b^{\circ} L$

Itt $L$

196'L

$99 t^{\circ} L$
$68 t^{\circ} L$

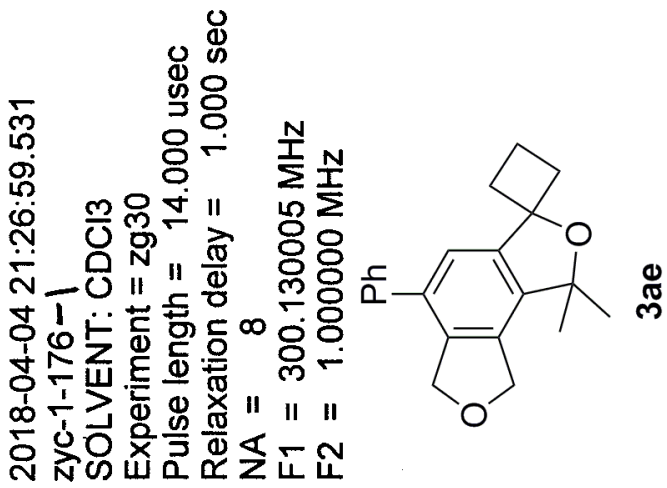

8 
$\dashv 98^{\circ} 8 \varepsilon$

$16 L^{\circ}$

EOl $\varepsilon$

$\angle L G^{\prime} 9$

$000^{\circ} \angle L$

घ己Ь $L L$

SOL 78

60198
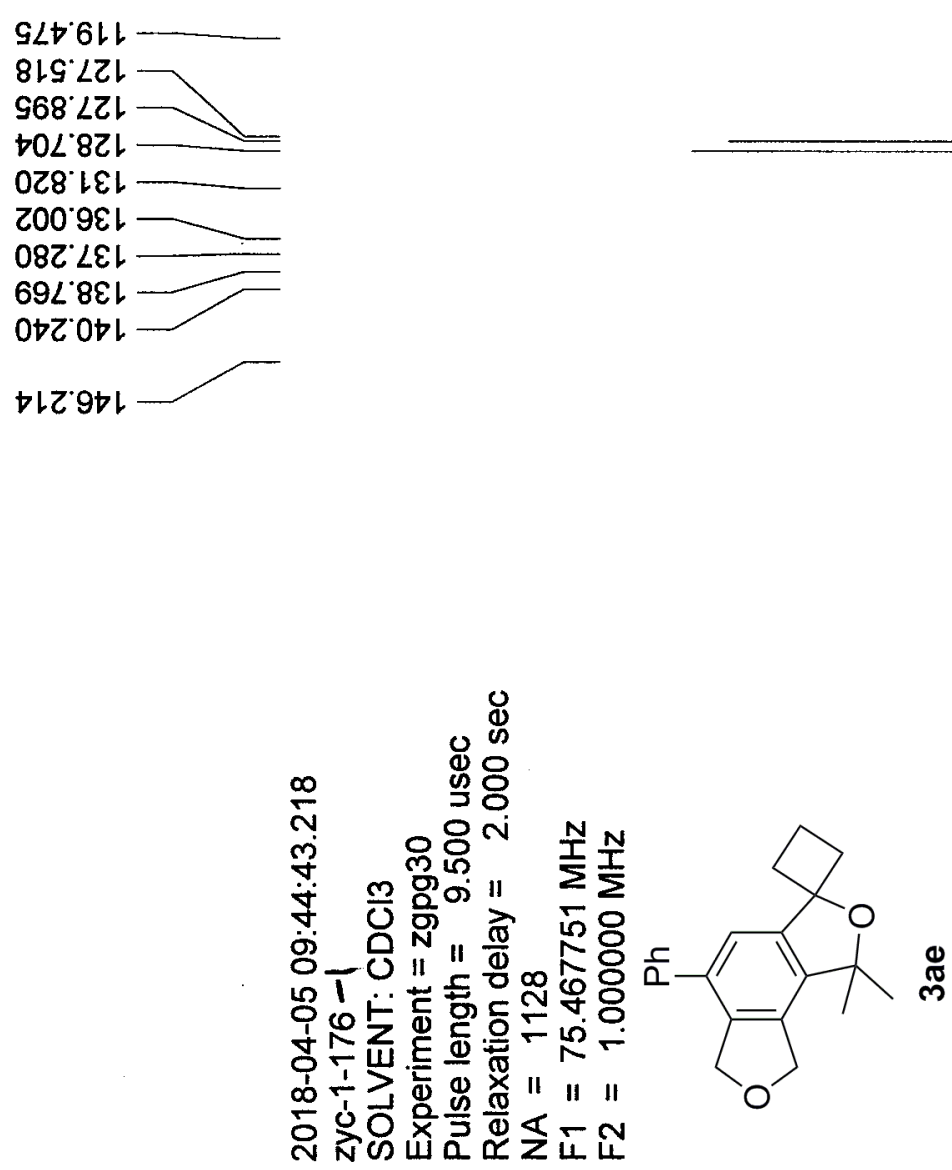

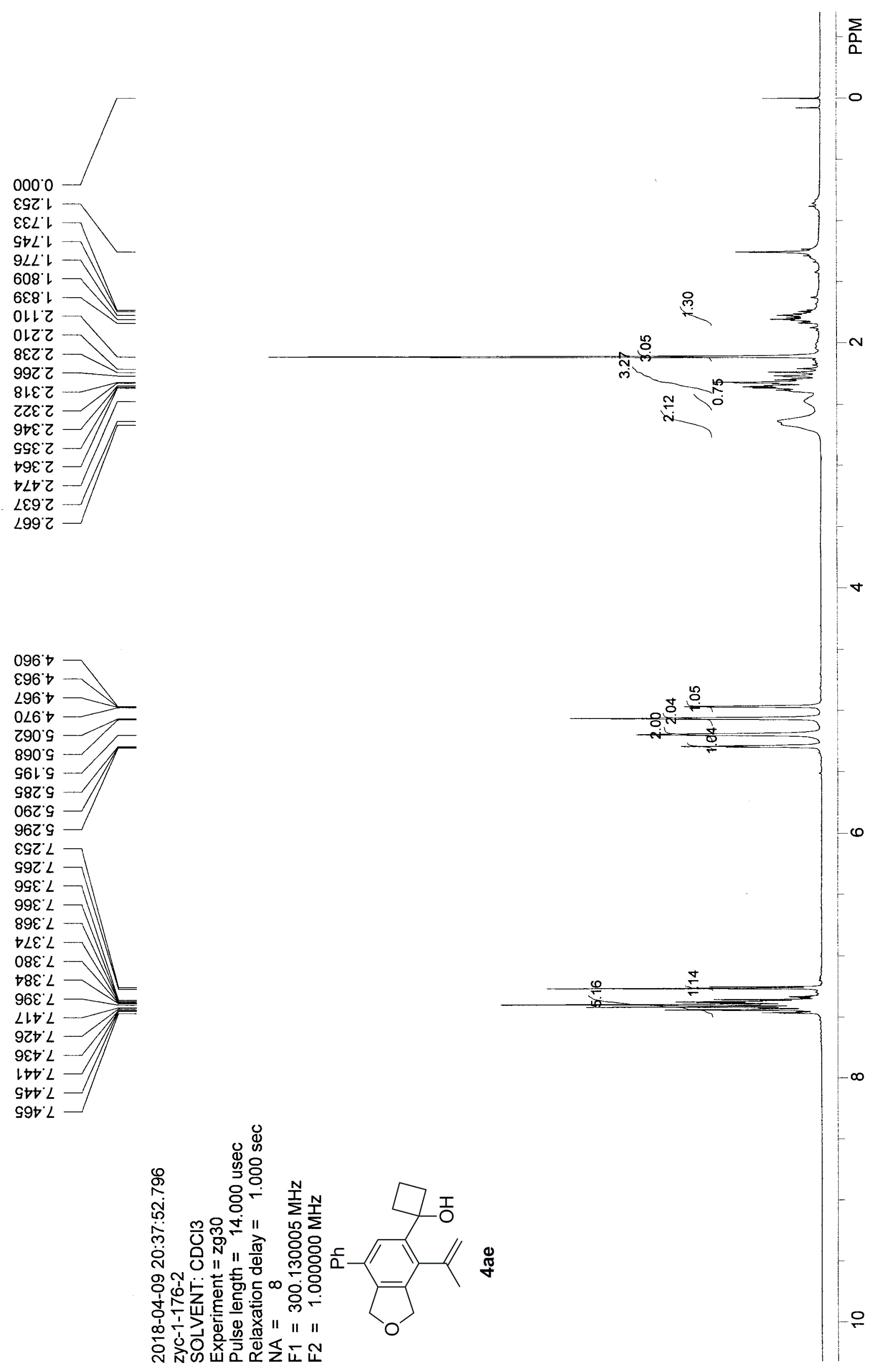
689 ' 1

$820^{\circ} \bullet Z$

$\angle 99^{\circ} 9 \varepsilon$

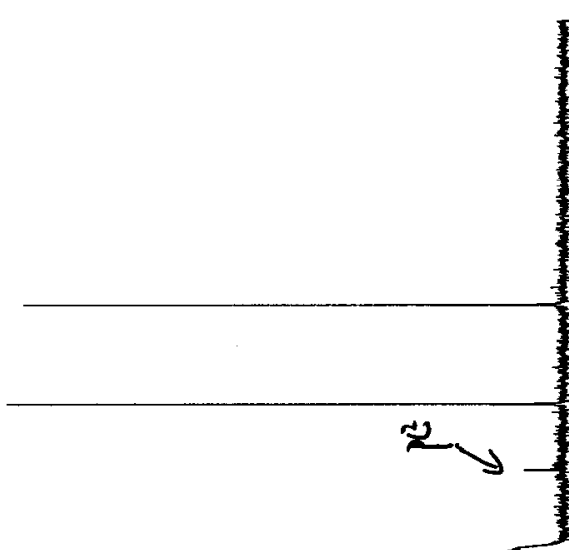

$990^{\circ} \varepsilon L$

$8 Z L \cdot E L$

LLG' $9 L$

$000^{\circ} \angle L$

$\varepsilon Z \nabla^{\circ} L L$

EZL $6 L$

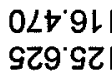

$Z \angle \nabla \angle Z L$

$\angle S L L Z$

9L9' 82

$\varepsilon 6 \mathrm{C}^{\circ} \triangleright \varepsilon$

OZL $9 \varepsilon$

$\succ 60.9 \varepsilon \downarrow$

$066.8 \varepsilon 1$

乙 $\angle 8^{\circ} 6 \varepsilon$

66l'Eり

699'tbl

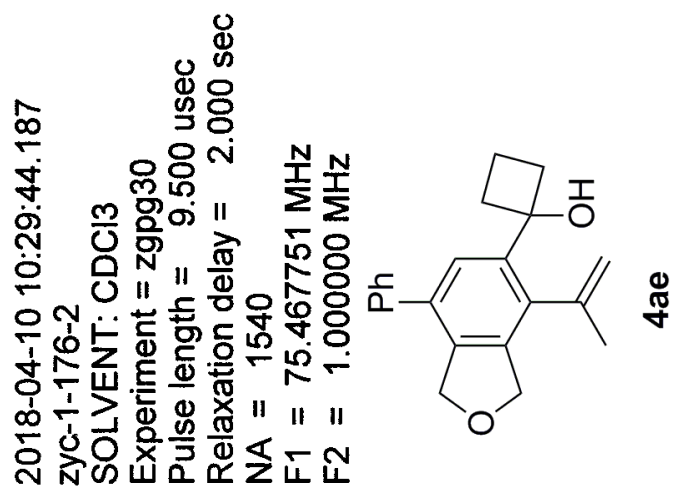



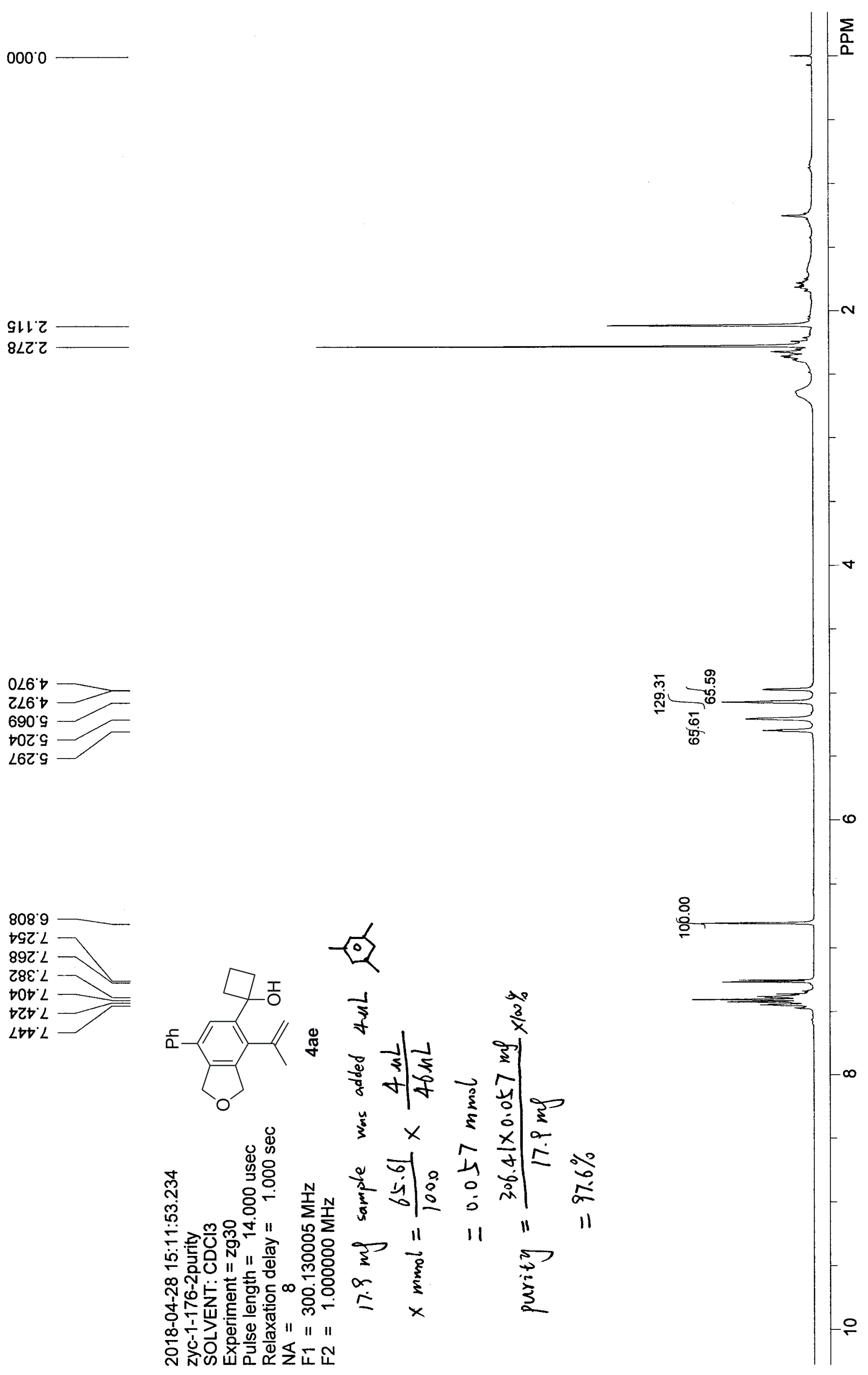

GIL

$8 \angle Z Z$ 

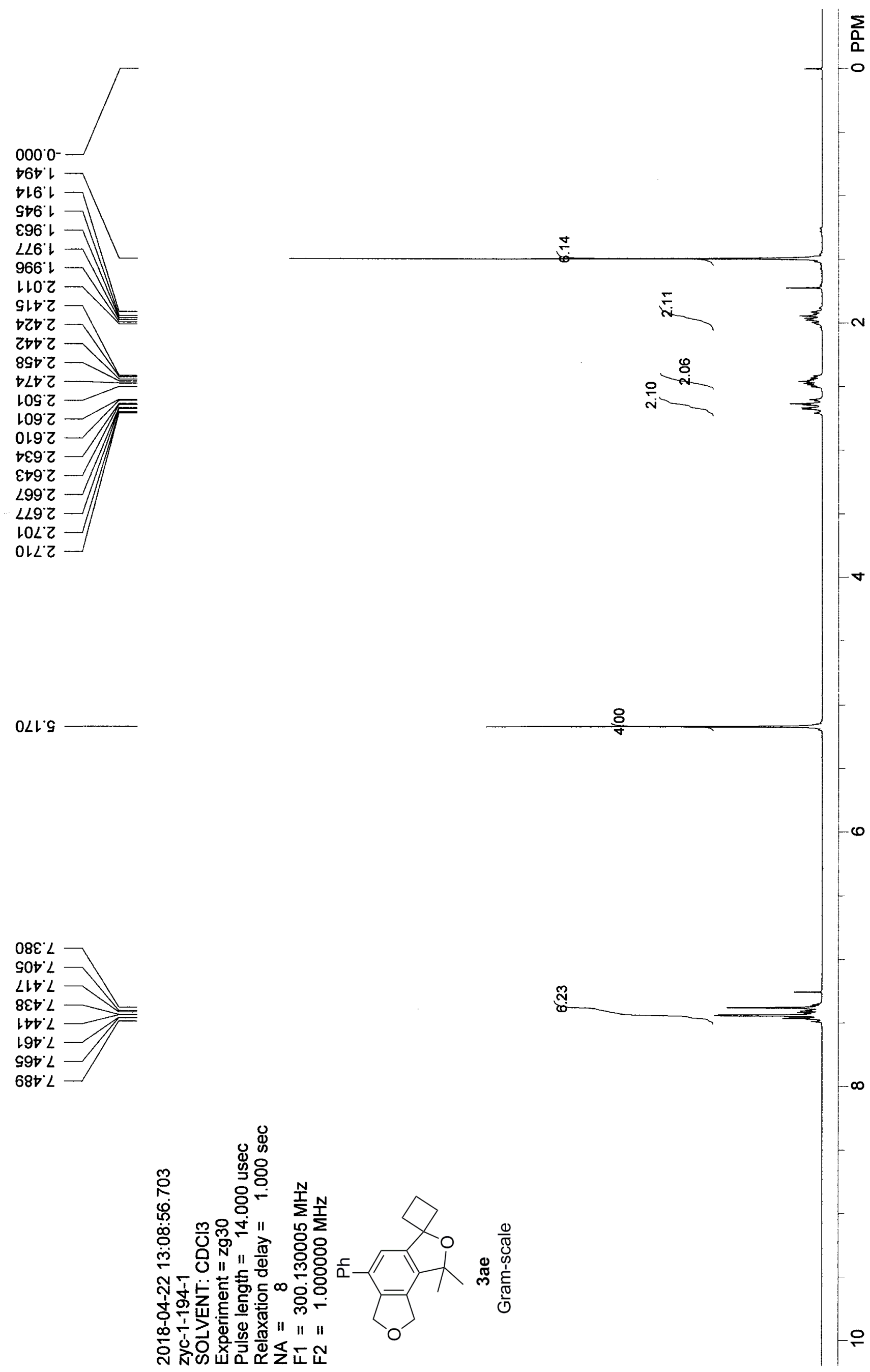

$0 \angle L^{\circ} 9$

จเ6.

St6.

ع96'

$\angle \angle 6^{\circ} \mathrm{L}$

$966^{\circ} \mathrm{L}$

1102

งเナZ

てヤヤて

89t 2

$\checkmark \angle D^{\circ} Z$

LOS 2

1092

OL9' $\mathrm{C}$

$\downarrow \varepsilon 9^{\circ} 乙$

$\varepsilon \triangleright 9^{\circ} 乙$

$\angle 99^{\circ} \mathrm{Z}$

$\angle \angle 9^{\circ} \mathrm{Z}$

$10 \angle 2$

OLLZ

$08 \varepsilon^{\circ} L$

SOt' $L$

$\angle L D L$

$8 \varepsilon+L$

$10+2$

$190^{\circ} \mathrm{L}$

S9t $L$

$680^{\circ} \mathrm{L}$ 
ฤตเยレ

$866^{\circ} \angle Z$

$9 \varepsilon 8^{\circ} 8 \mathcal{E}$

†SL

$990^{\circ} \varepsilon$

$899^{\circ} 9$

$000^{\circ} L L$
$\varepsilon Z \nabla^{\circ} L L$

$890^{\circ} \vee 8$

$001 \cdot 98$
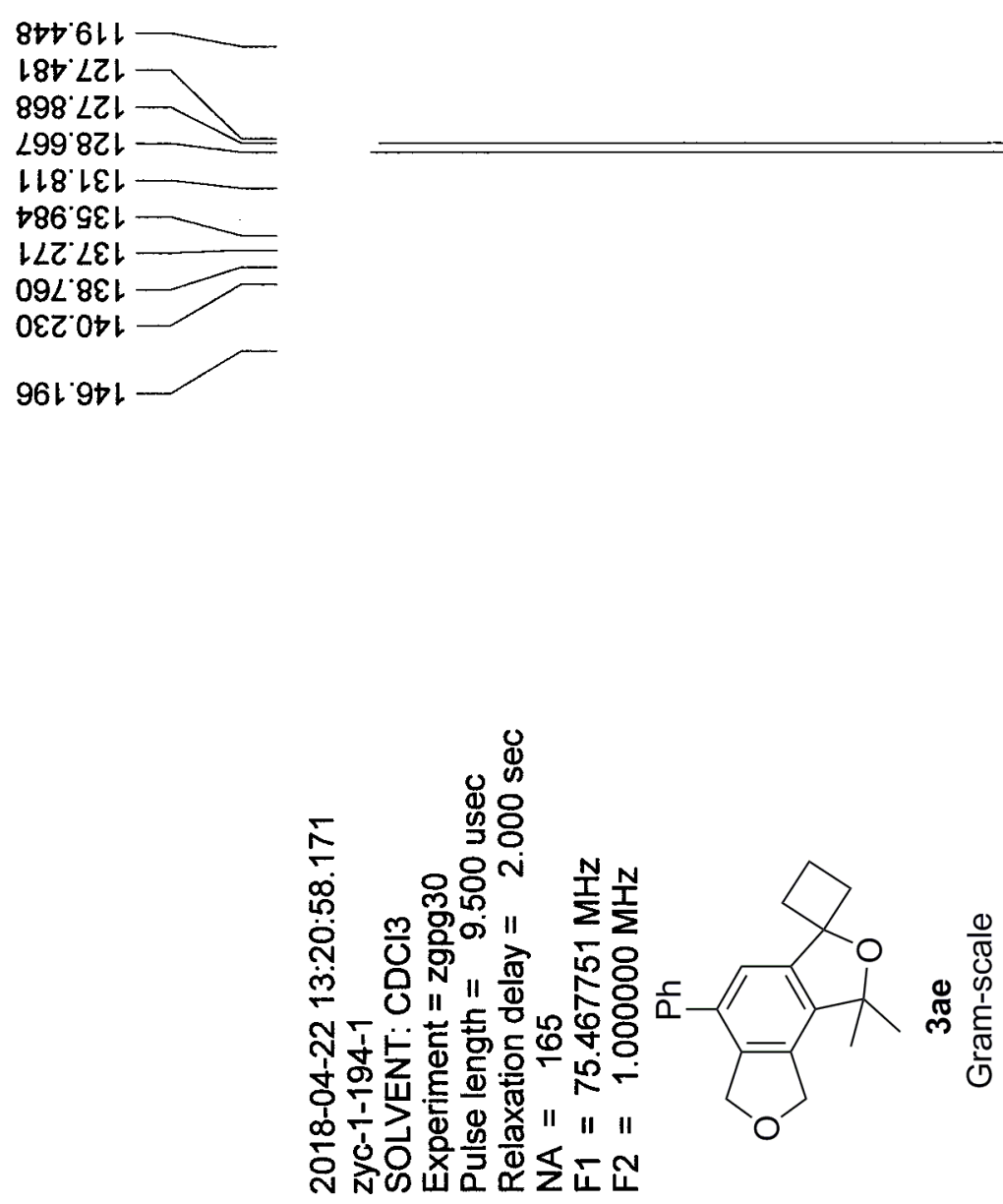
$000^{\circ} 0^{-}$
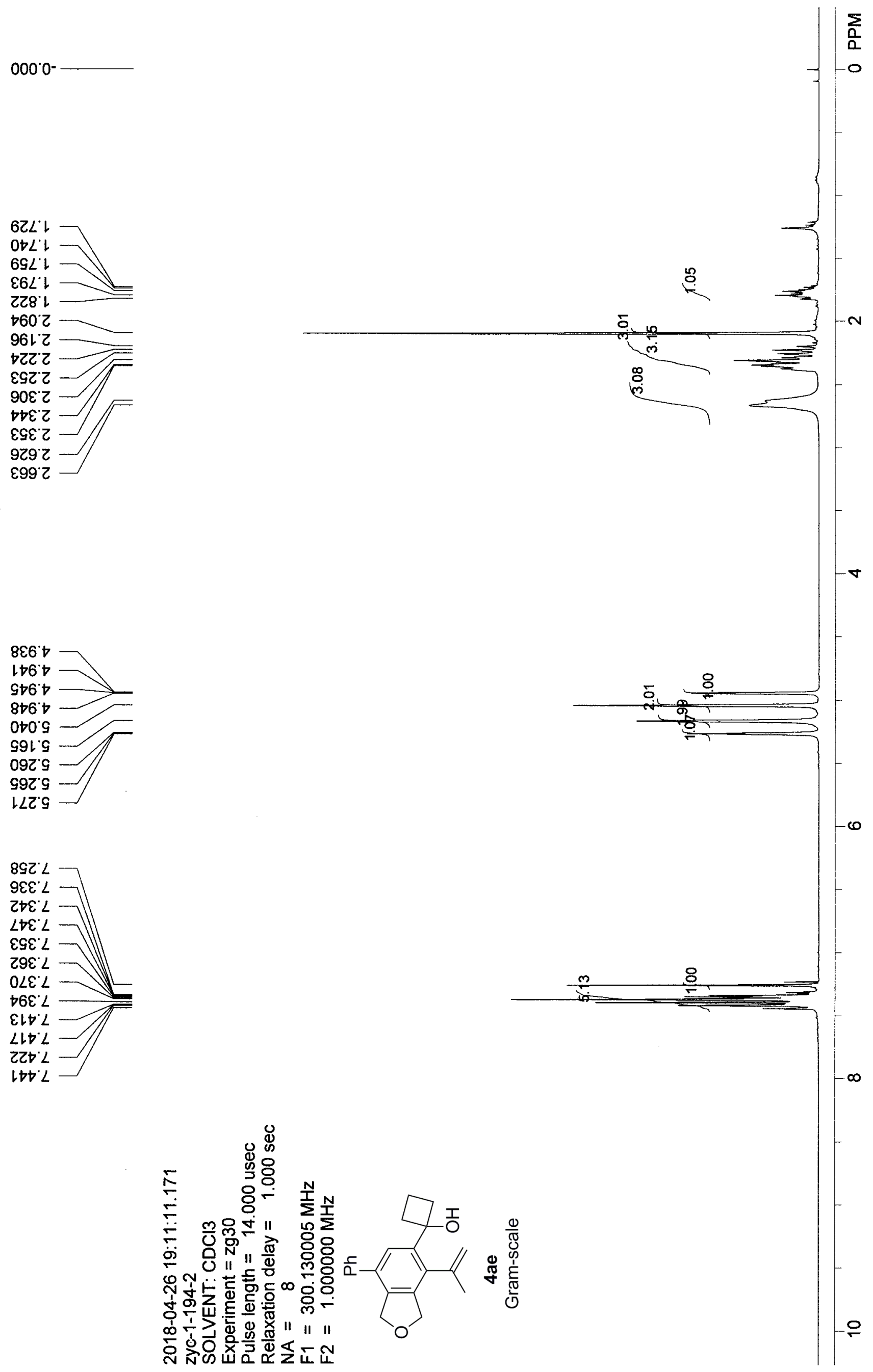

8\&6't

It6 $t$

8เ6.

$0+0 \mathrm{~s}$

S9l's

0929

S9Z' 9

LLZ'G

$892 L$

$9 \varepsilon \varepsilon ' L$

乙† ' $L$

$\angle \downarrow E \cdot \angle$

¿SE $L$

乙9\&' $L$

$0 \angle \varepsilon L$

$\nabla 6 \varepsilon<$

Elti $L$

$\angle L D^{\circ} L$

ZCD 2

Lt $L$ 
$\vdash \varepsilon \mathcal{G}^{\prime} \mathrm{l}$

$\varepsilon 96$ ' $\varepsilon 乙$

6 เو $9 \varepsilon$

$\varepsilon 86^{\circ} \mathrm{LL}$

$\angle Z 9^{\circ} \varepsilon L$

$\angle \angle G^{\circ} 9 L$

$000 \% L$

$\varepsilon Z \nabla^{\circ} L L$

S86 82

0૬ร'9

889'GZL

$66 \varepsilon^{\prime} \angle Z$ L

$\rightarrow \angle 9^{\circ} \angle Z L$

219.8ZL

$\varepsilon L L \triangleright \varepsilon$

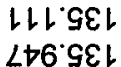

$0 \angle 8^{\circ} 8 \varepsilon L$

$808^{\circ} 6 \varepsilon$ L

06! E๐

ててナ゙ャレ

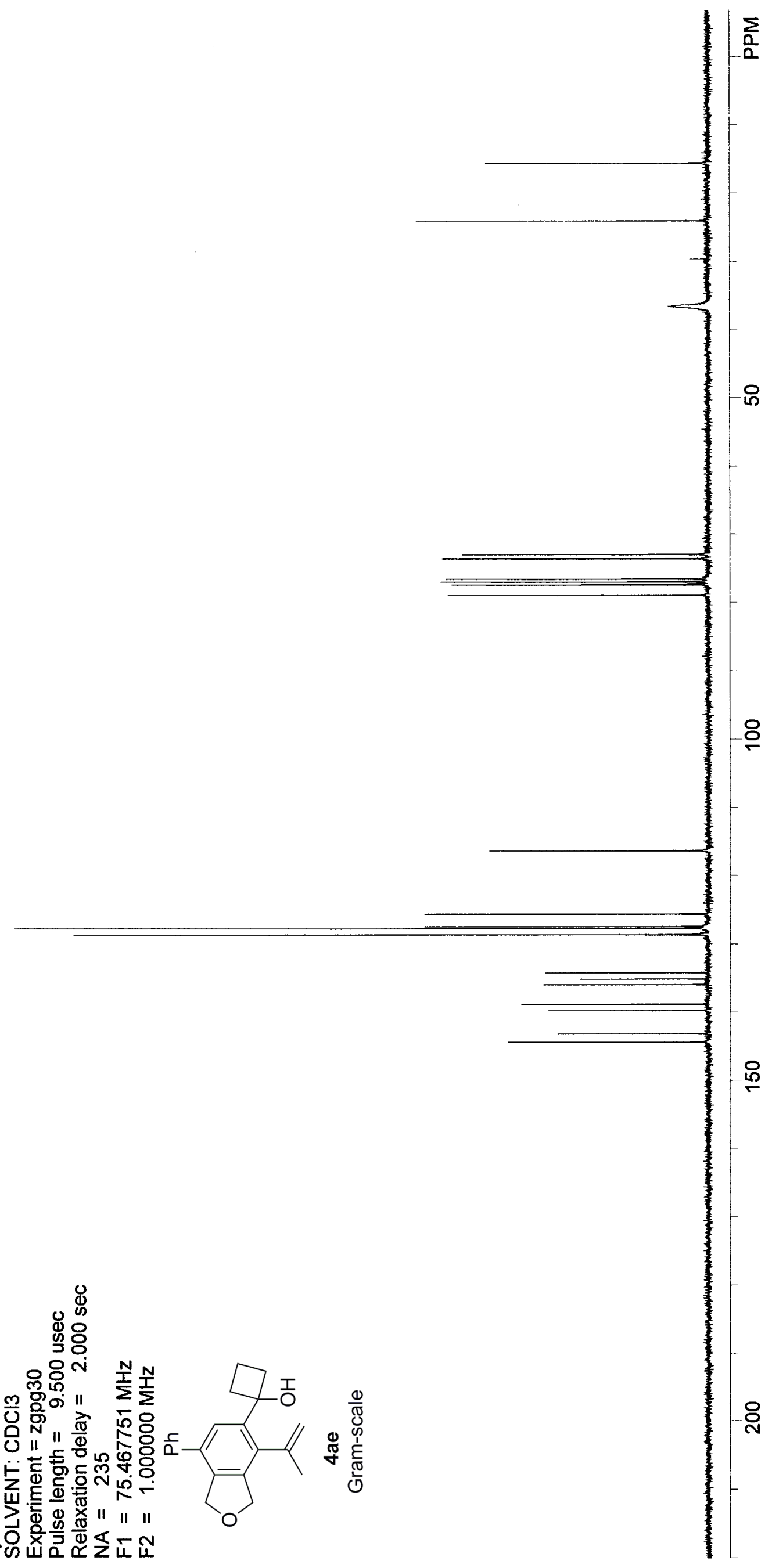



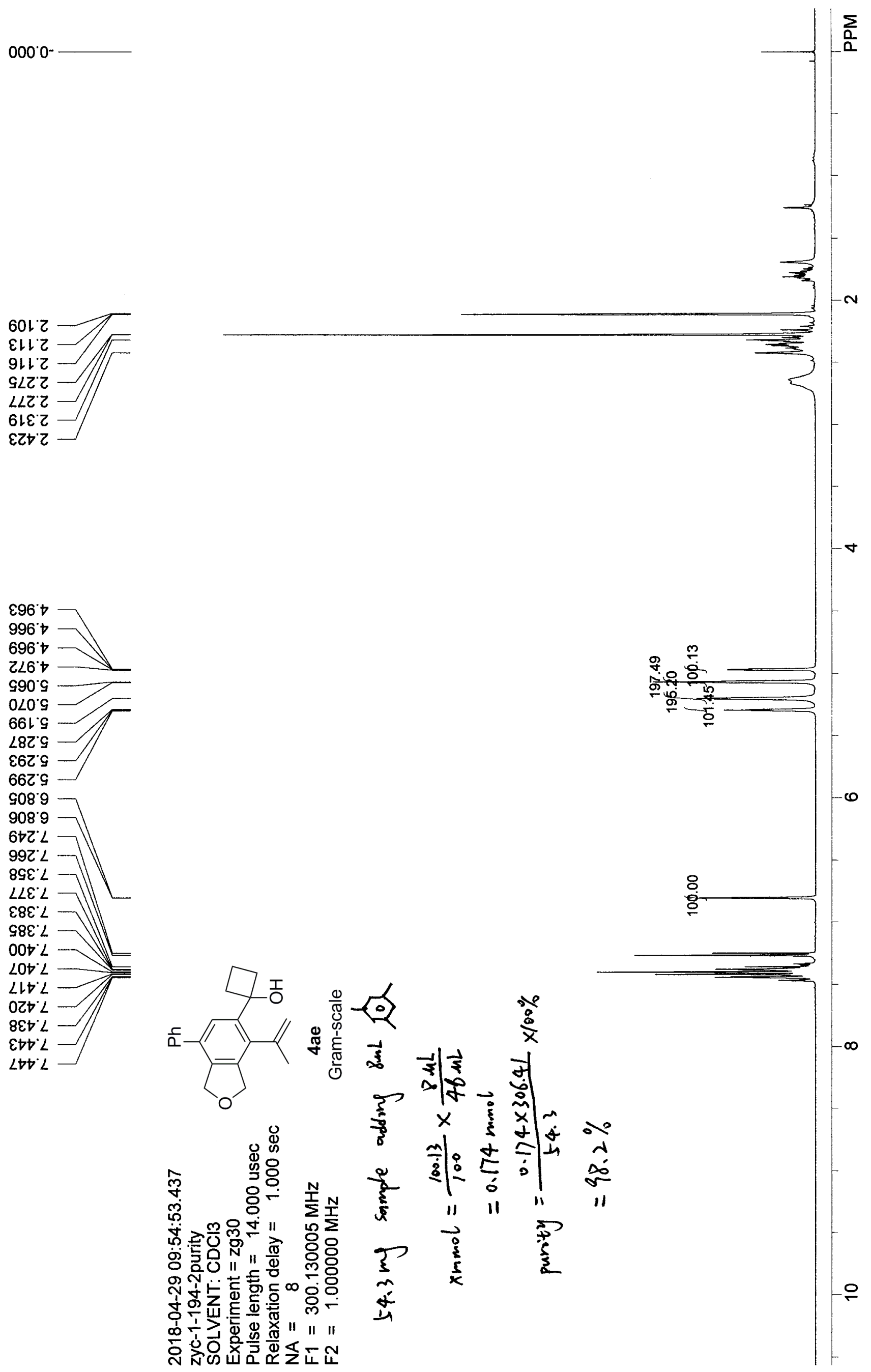


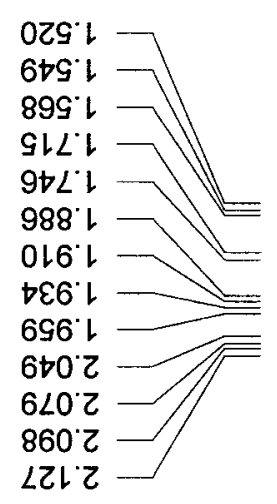

$\left\{\begin{array}{l}\sum_{0} \\ -8\end{array}\right.$

$291 \cdot 9$

s9.' 9

$891^{\circ} \mathrm{G}$

$6 \mathrm{LI} \mathrm{S}$

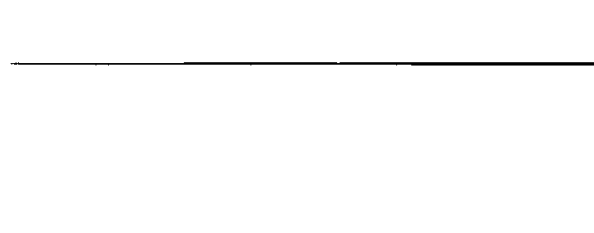

움
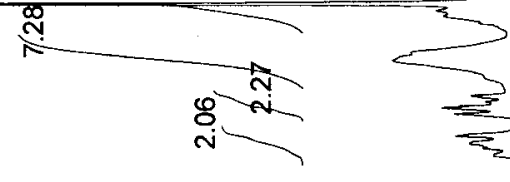

8
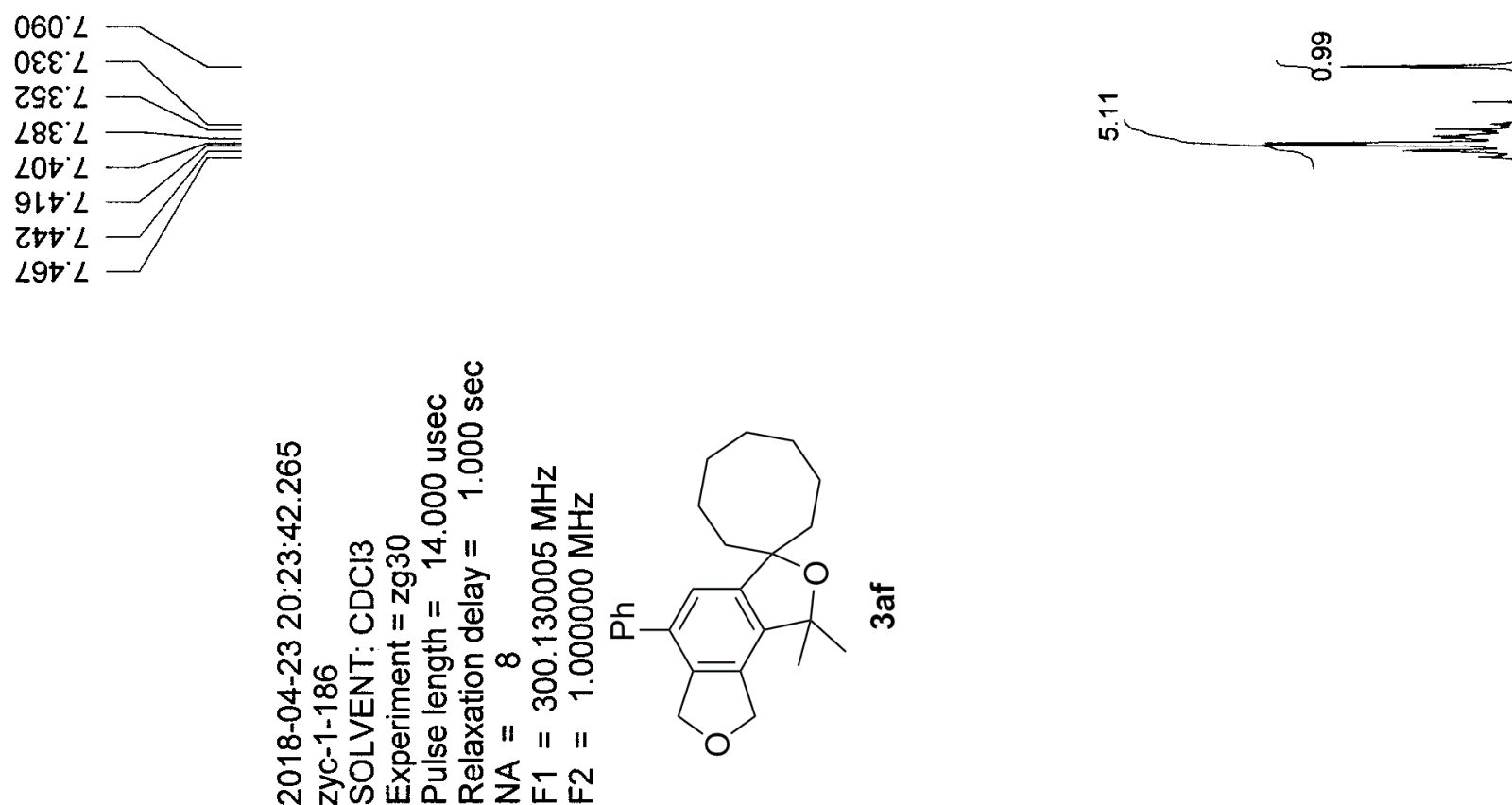
$8 \angle 9^{\circ} 8 \varepsilon$
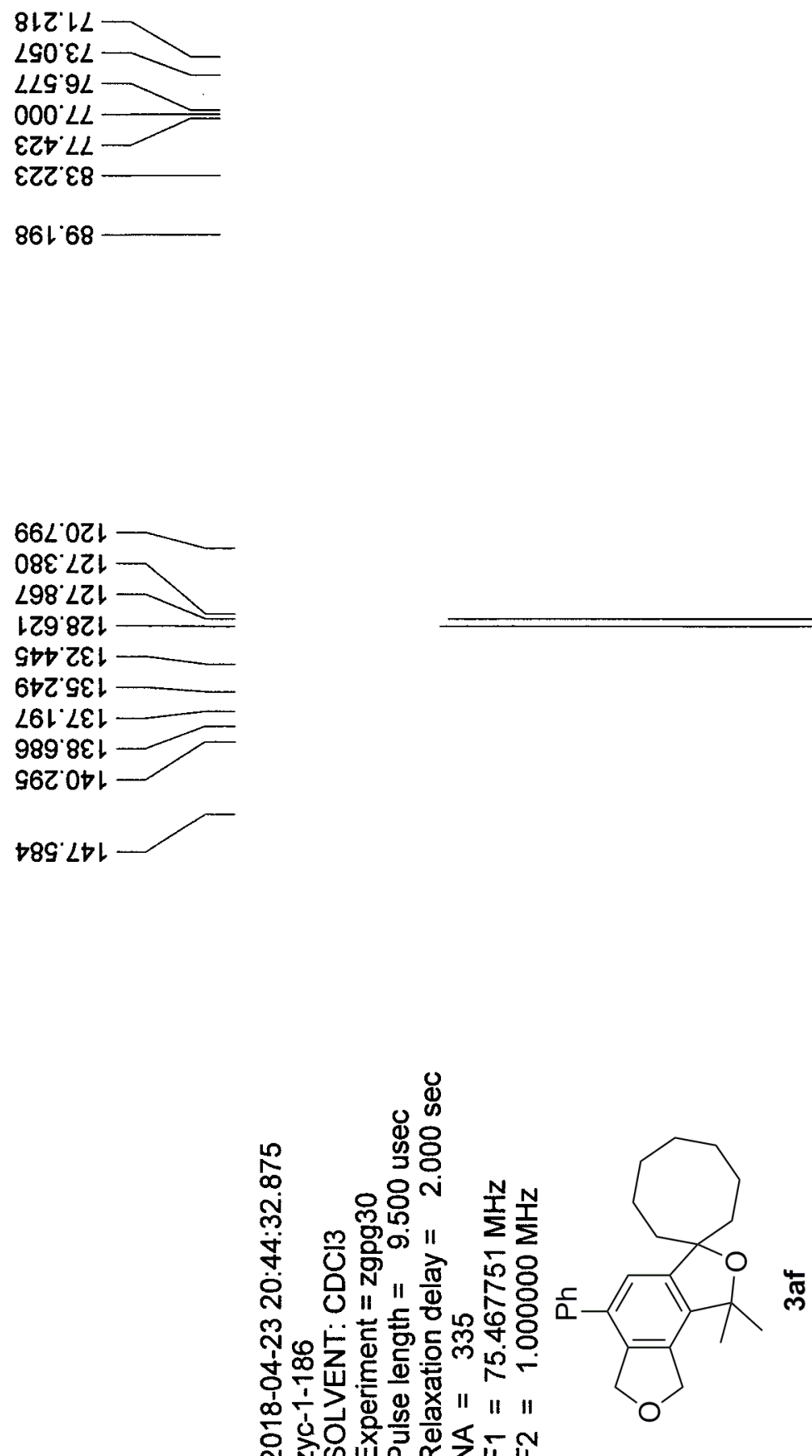
bIt

819'

OEL'L

IGL'L

$9 \angle L L$

$108^{\circ} \mathrm{L}$

628 !

998'

$\varepsilon 88^{\prime}$.

$806^{\circ} \mathrm{L}$

$8 Z 6^{\prime}$ '
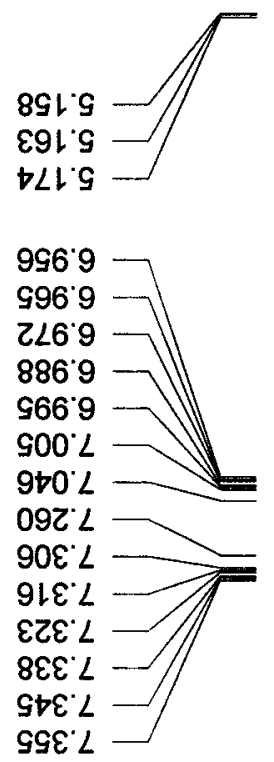

$\longrightarrow\left\{\frac{\Sigma}{n}\right.$

$\stackrel{\infty}{\circ}$

8

స̃ $=\stackrel{9}{\circ}$

홍

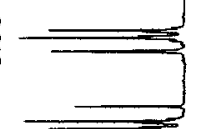

$\stackrel{f}{q}$
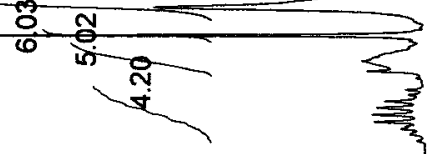

$N$

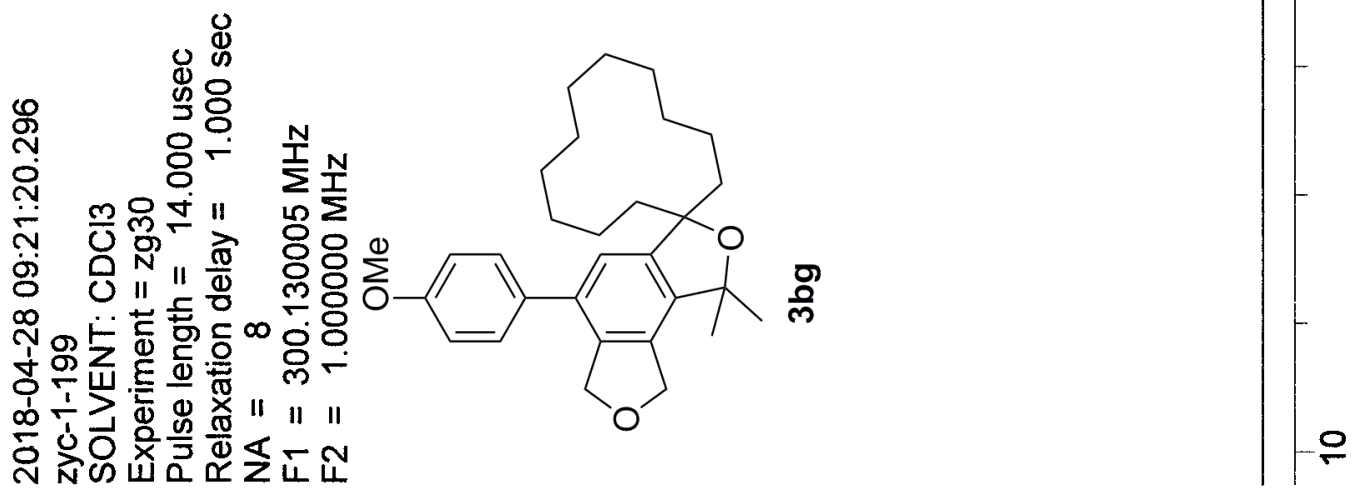


$006^{\circ} 6 \mathrm{~L}$

9ZZ टZ

$899^{\circ} \mathrm{ZZ}$

896.92

9tG' 92

S90 62

ᄂ.9.9E

08299

†9Z LL

$89 L^{\circ} \varepsilon$

$\angle \angle G 9 L$

$000^{\circ} \angle L$

EZt $L L$

乙๖६ $\varepsilon 8$

86168

๑ย๐ャレレ

てレレレレ

$866^{\circ} 8 \mathrm{Z}$ L
$\nabla \triangleright \varepsilon^{\prime} 乙 \varepsilon\llcorner$

$\succ 89$ ¿ह।

$\varepsilon \nabla L ' \nabla \varepsilon$

$\angle 96$ ' $9 \varepsilon$

$9 \varepsilon \mathcal{C}^{\prime} 8 \varepsilon\llcorner$

20L'9t

L66.89L

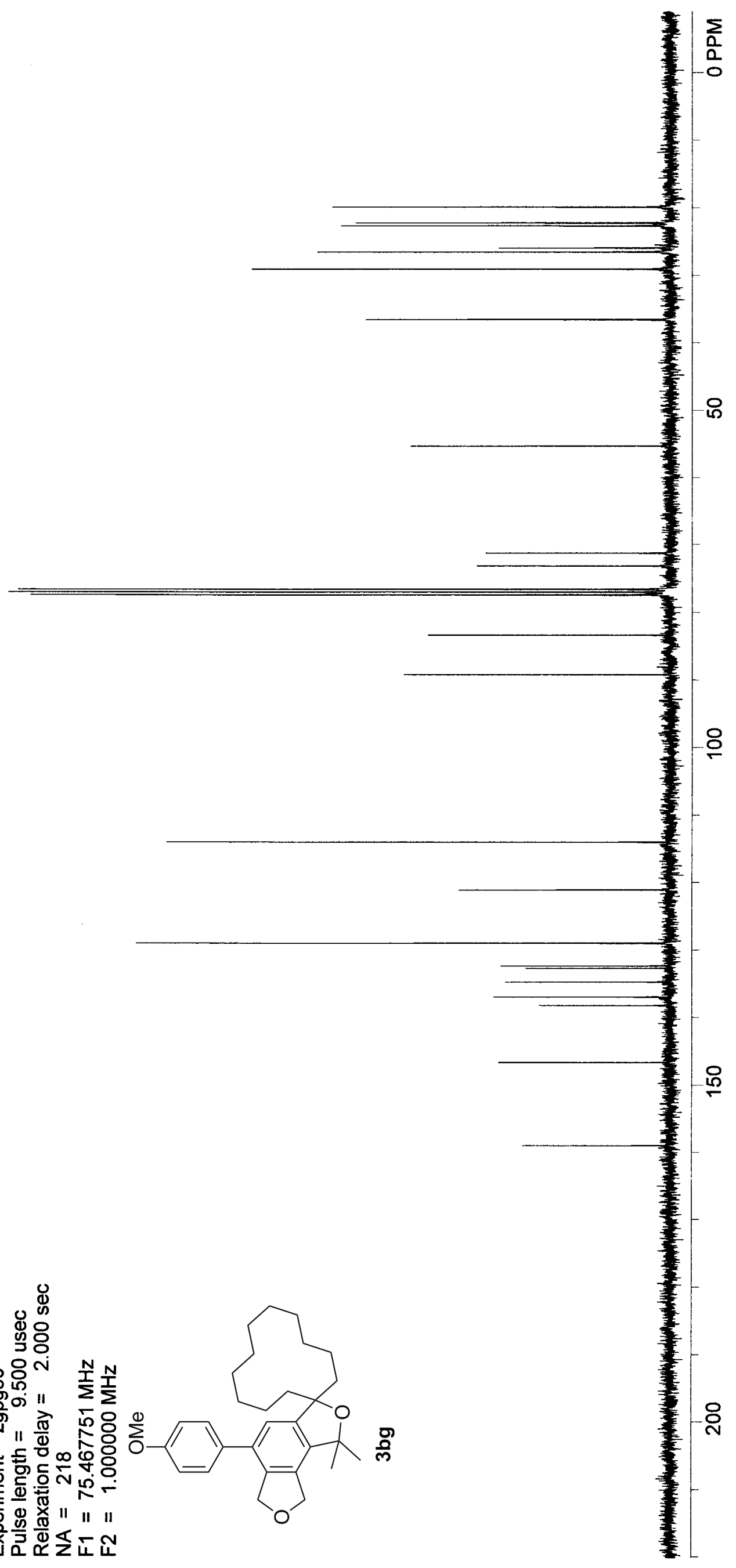


$000^{\circ} 0$
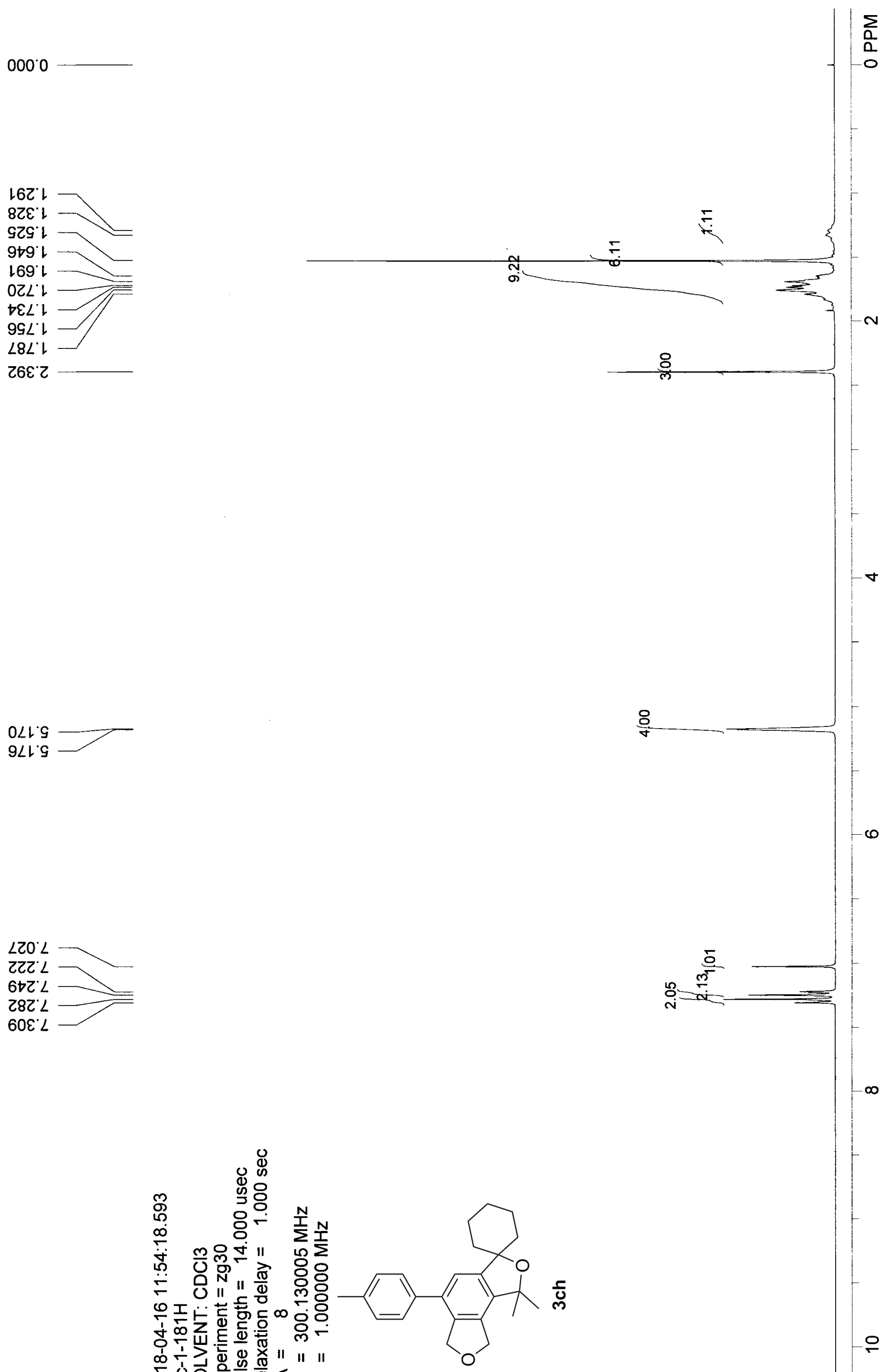
$960^{\circ} に 2$

$9 \varepsilon \varepsilon^{\circ} 乙 Z$

เЬZ 92

$8 \varepsilon l^{\circ} 62$

$869^{\circ} 6 \varepsilon$

$281^{\circ} \mathrm{LL}$

OEL $E L$

$\angle L G^{\prime} 9 \angle$

$000^{\circ} L L$

\&乙† $L L$

ยะย $\varepsilon 8$

$\angle L 9^{\circ} 98$

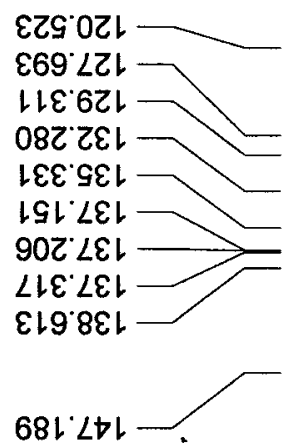

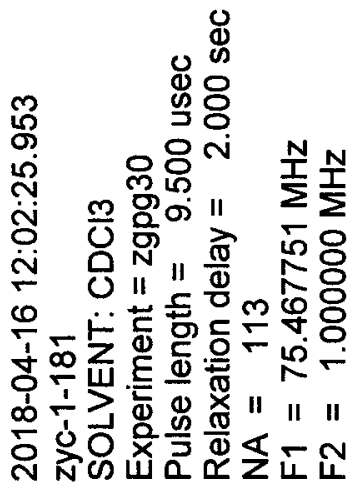

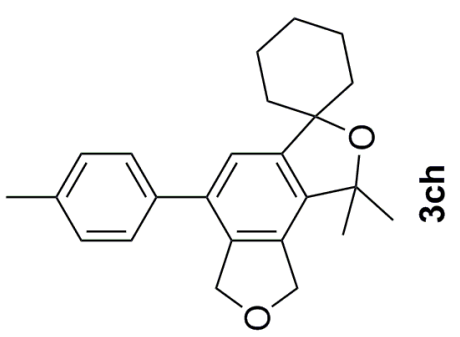

잉

움 

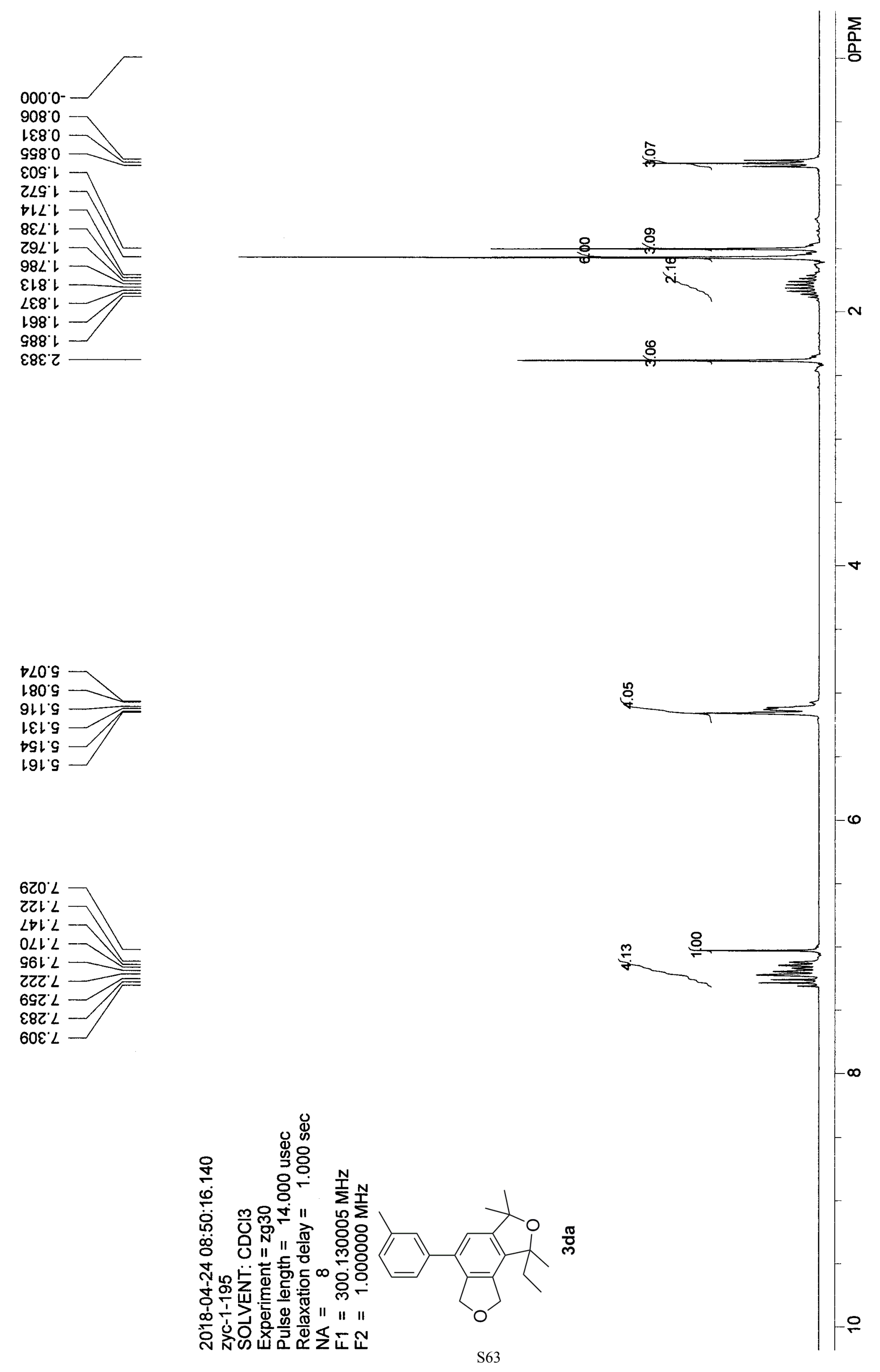
SIZ'L

S69 LZ

$189^{\circ} 62$

†ะट $\llcorner\varepsilon$

เ०LZE
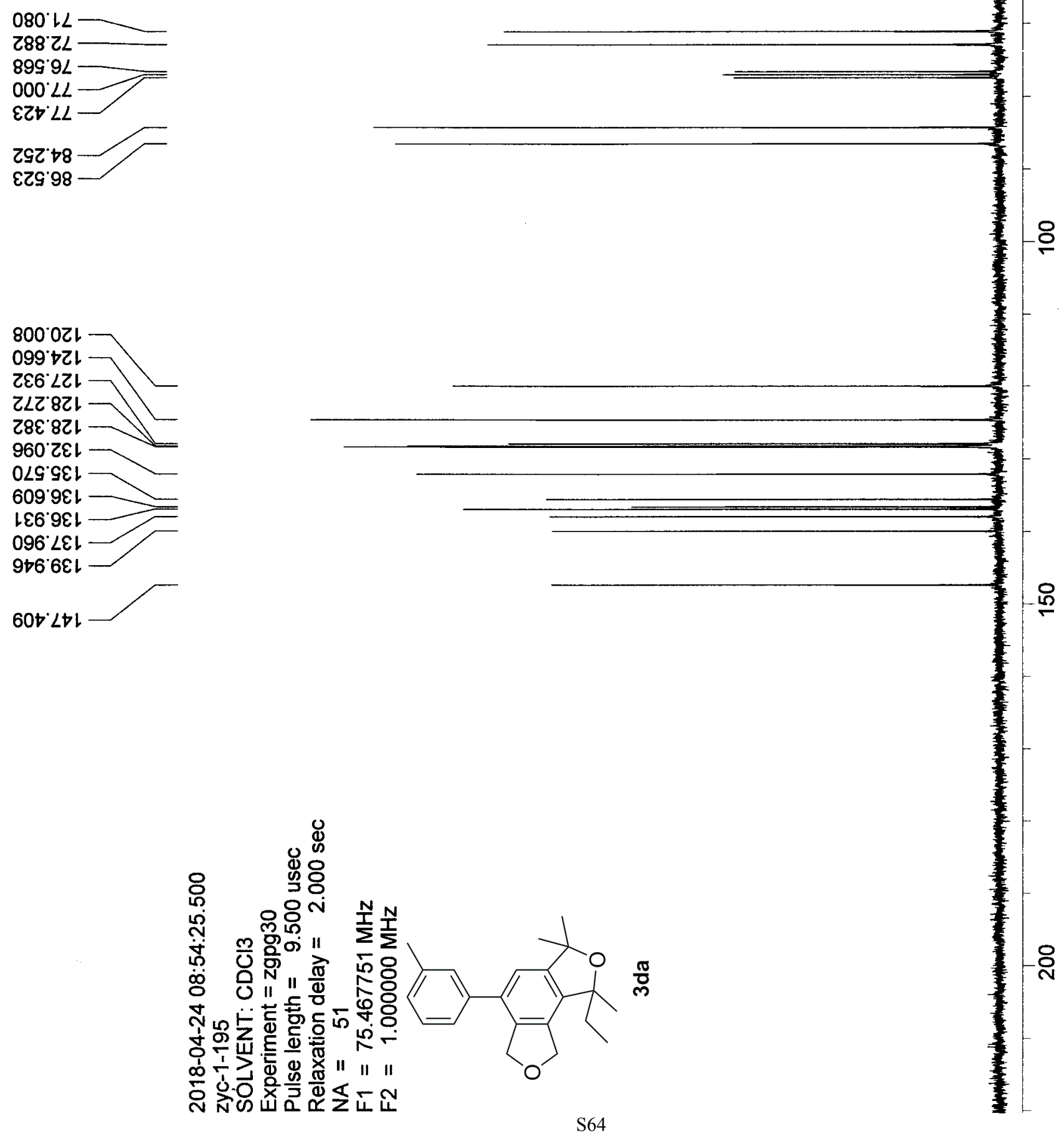
EGट !

$\angle 6 Z$

†ह'।

LZG'

ES9'

9L9'

$169^{\prime} \mathrm{L}$

8LL'

$\checkmark G L '$

$88 L !$

$628^{\prime}$ ᄂ

$\left\{\begin{array}{l|l}\sum & \sum \\ 0 \\ 0 \\ -0\end{array}\right.$

GZL'

IEL'

$6 \varepsilon+9$

$\angle L L G$

E81' 9

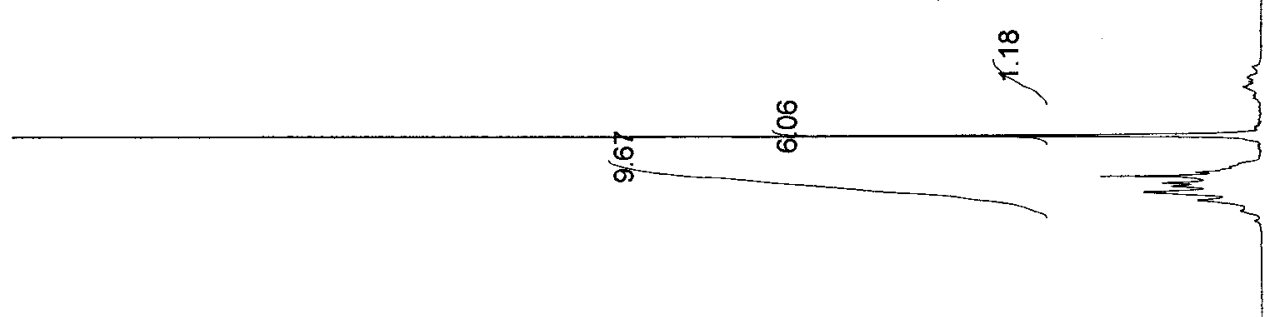

$-N$

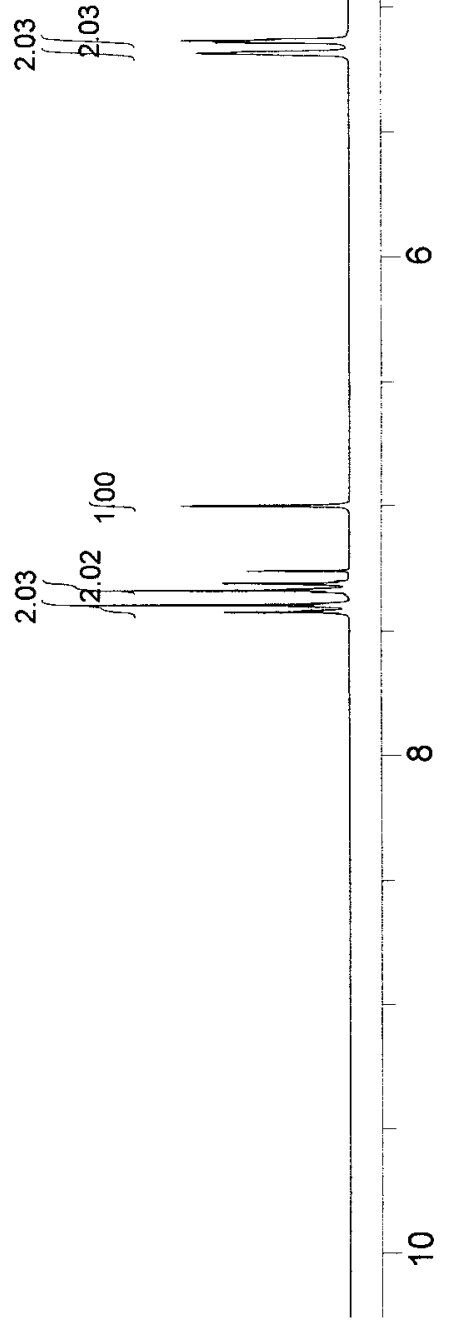

옥

$000^{\circ} \mathrm{L}$

OLE L

$6 \varepsilon \varepsilon^{\circ} L$

$96 \varepsilon \angle$

GZ†'L 
$9 \varepsilon \varepsilon \mathrm{ZZ}$

เ৮Z $9 Z$

02162

$989^{\circ} 6 \varepsilon$

$60 Z$ L

$\varepsilon 86.2 L$

$\angle L G^{\prime} 9 L$

$000 \% L$

$\varepsilon Z \nabla L L$

$88 \varepsilon^{\circ} \varepsilon 8$

SOL'G8
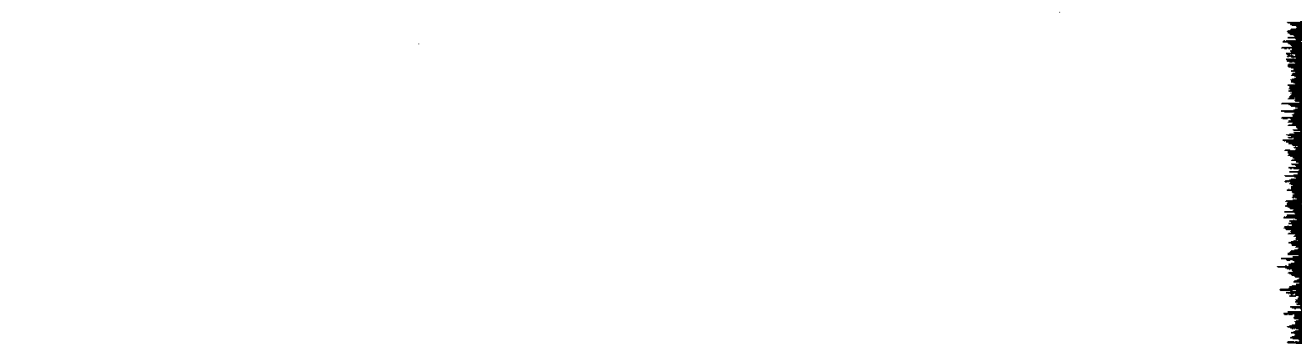

SOS.0ZL

$\varepsilon \varepsilon 8.8 Z$

เ9l.6Zl

S9G'ZहL

SLt $\varepsilon \varepsilon \downarrow$

Z8เ $\bullet \varepsilon L$

$86 Z \angle \varepsilon L$

$9898 \varepsilon$

เレย $6 \varepsilon เ$

$9 t b<b l$

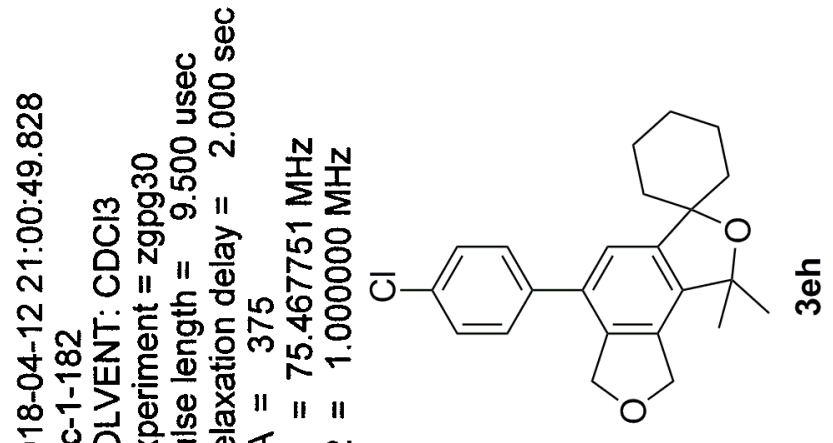

क.

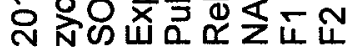



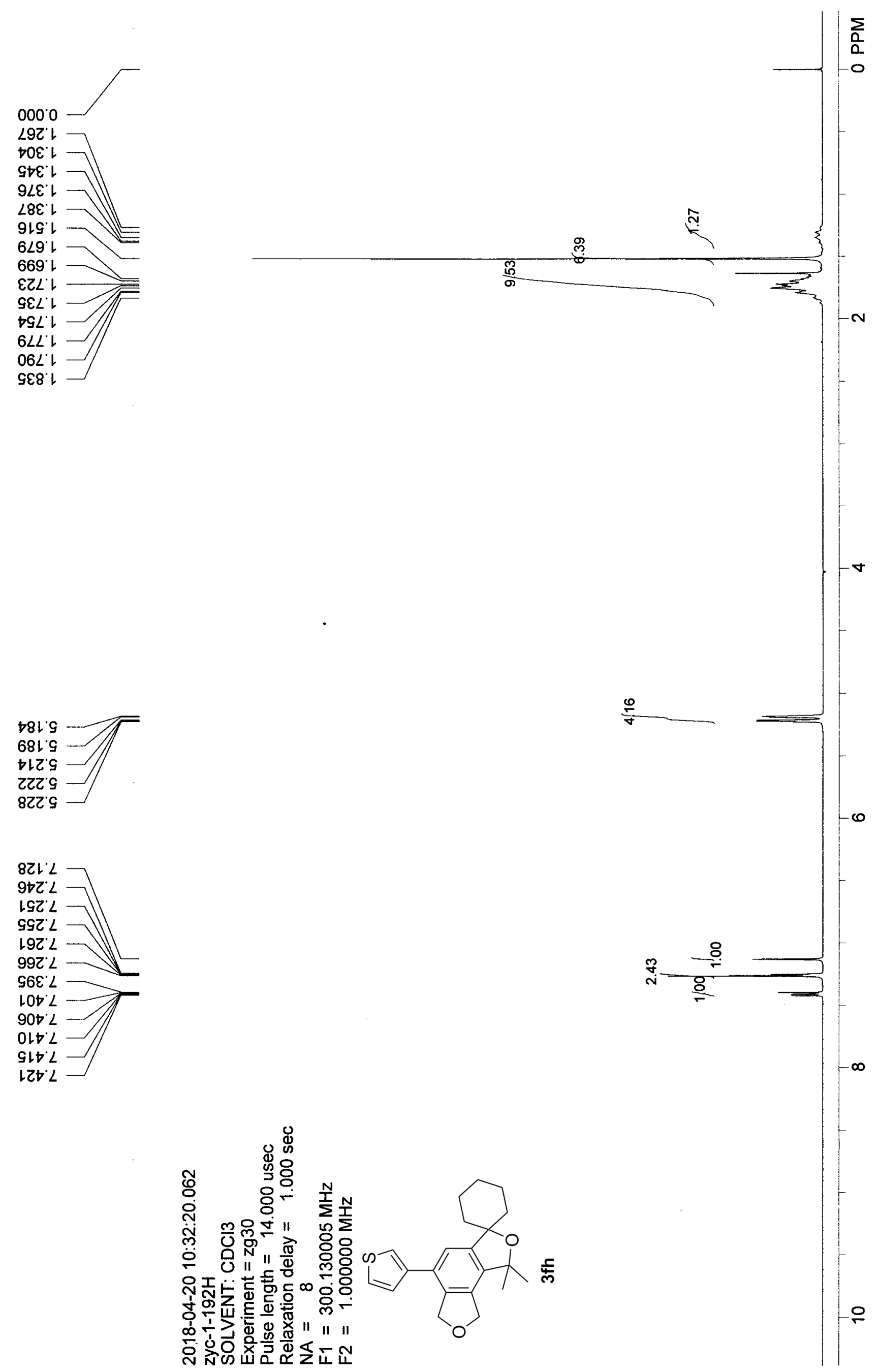
$08 \mathcal{Q}^{\circ} 6 \varepsilon$

ZLLL

$0 \angle t^{\circ} \varepsilon L$

$\angle L G^{\prime} 9 L$

$000^{\circ} \mathrm{LL}$

$\varepsilon Z \nabla^{\circ} L L$

\section{乙†ย' $\varepsilon 8$}

$229^{\circ} 98$

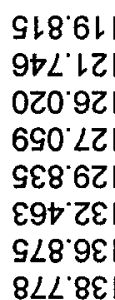

$8 \angle L 8 E L$
$\forall ヤ 9^{\circ} 0 \triangleright 1$

$68 L^{\prime} \angle t \downarrow$

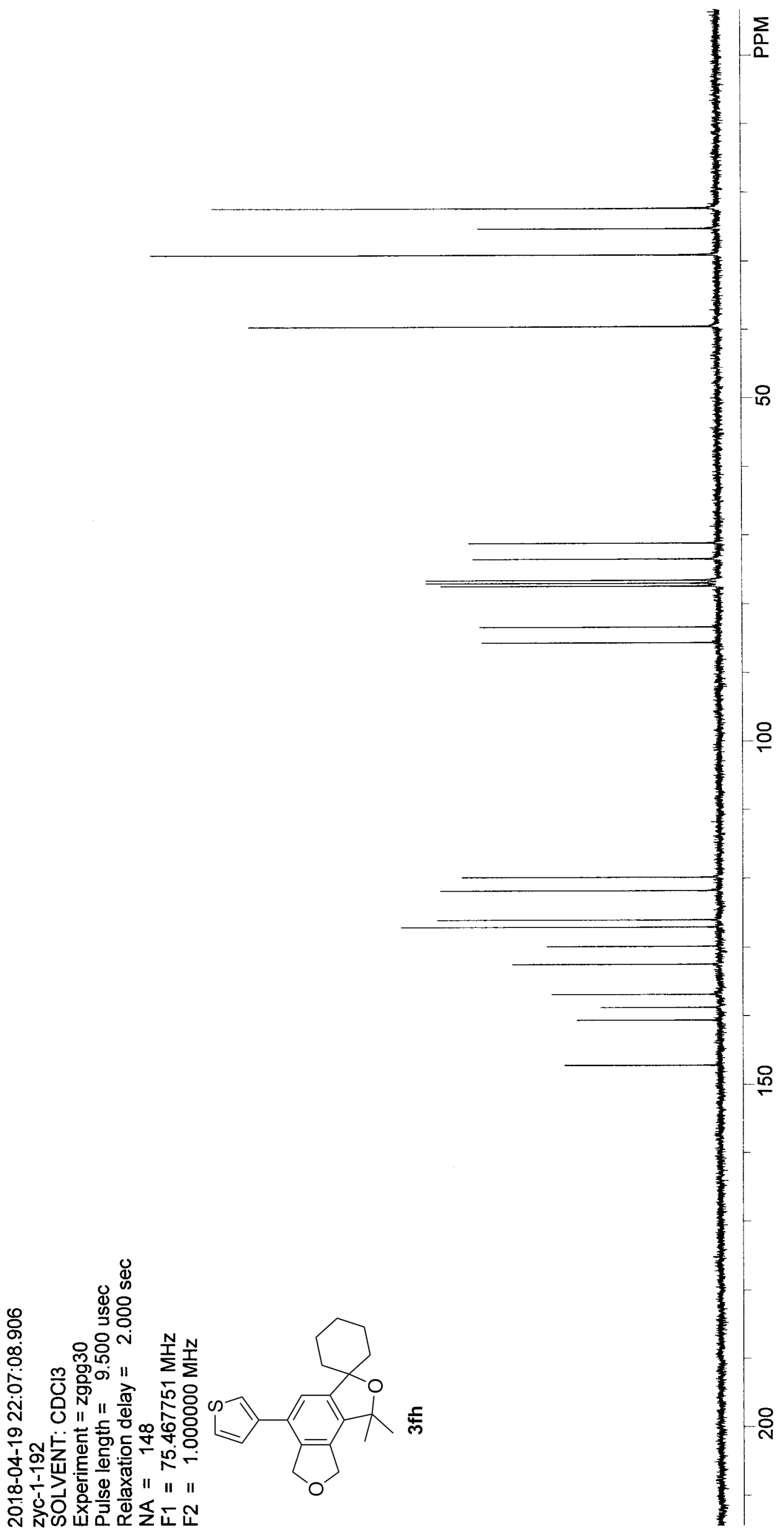



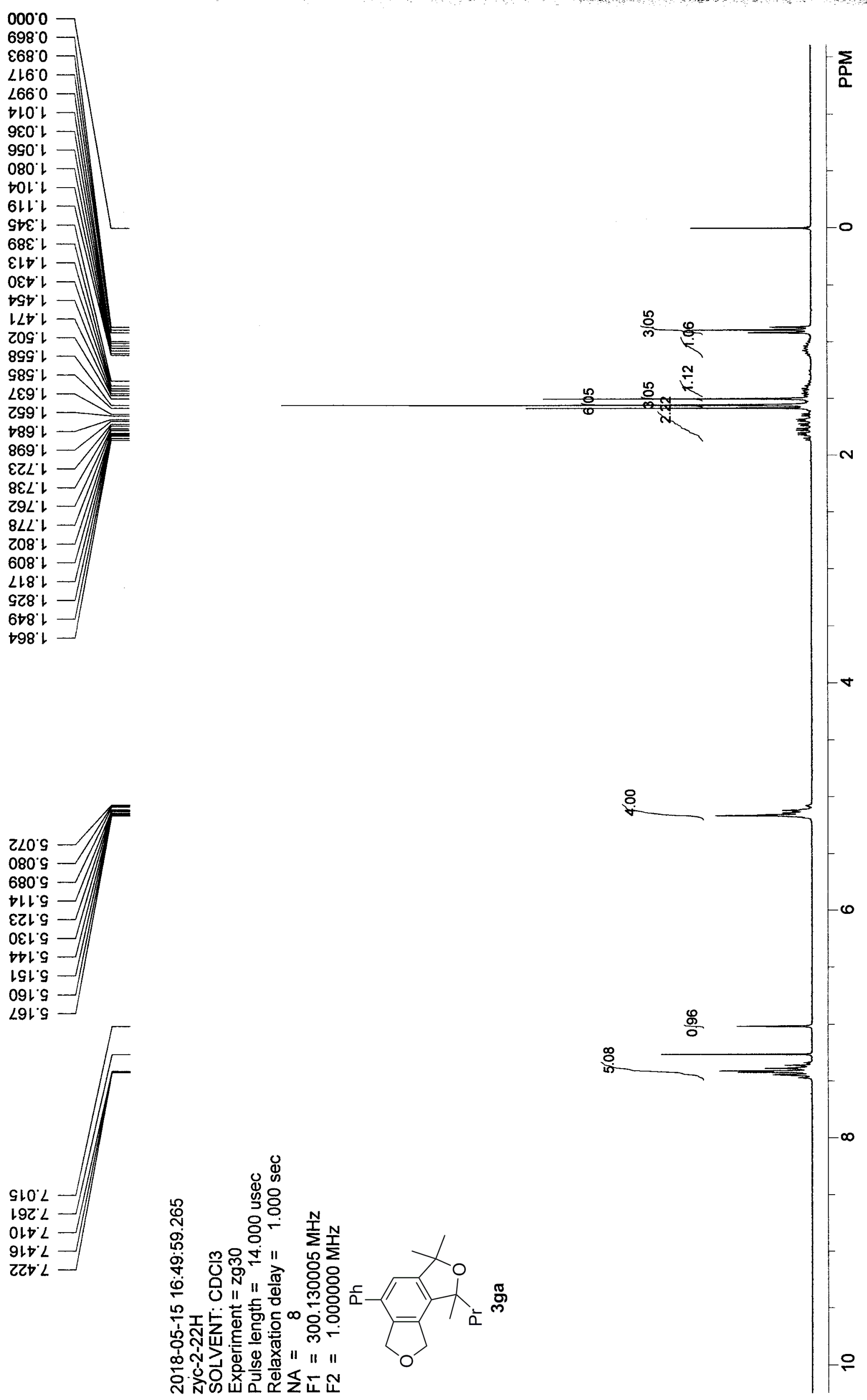
6จยเレ

TL8LL

$\angle E Z^{\prime} 8 Z$

106.62

LZ†

$\varepsilon \varepsilon\llcorner 乙 \succ$

9SEレL

ㄴ. $\varepsilon L$

$\angle \angle G^{\prime} 9 \angle$

$000^{\circ} L L$

$\varepsilon Z \nabla L L$

เ6เจ8

$98+98$
GLZOZ

80t LCL

CL8 $\angle Z L$

21982

08Z ZहL

689' $9 \varepsilon$

$8 \angle 0 \angle E L$

ZSZ $\angle E L$

LGLODL

$629 \angle \nabla L$

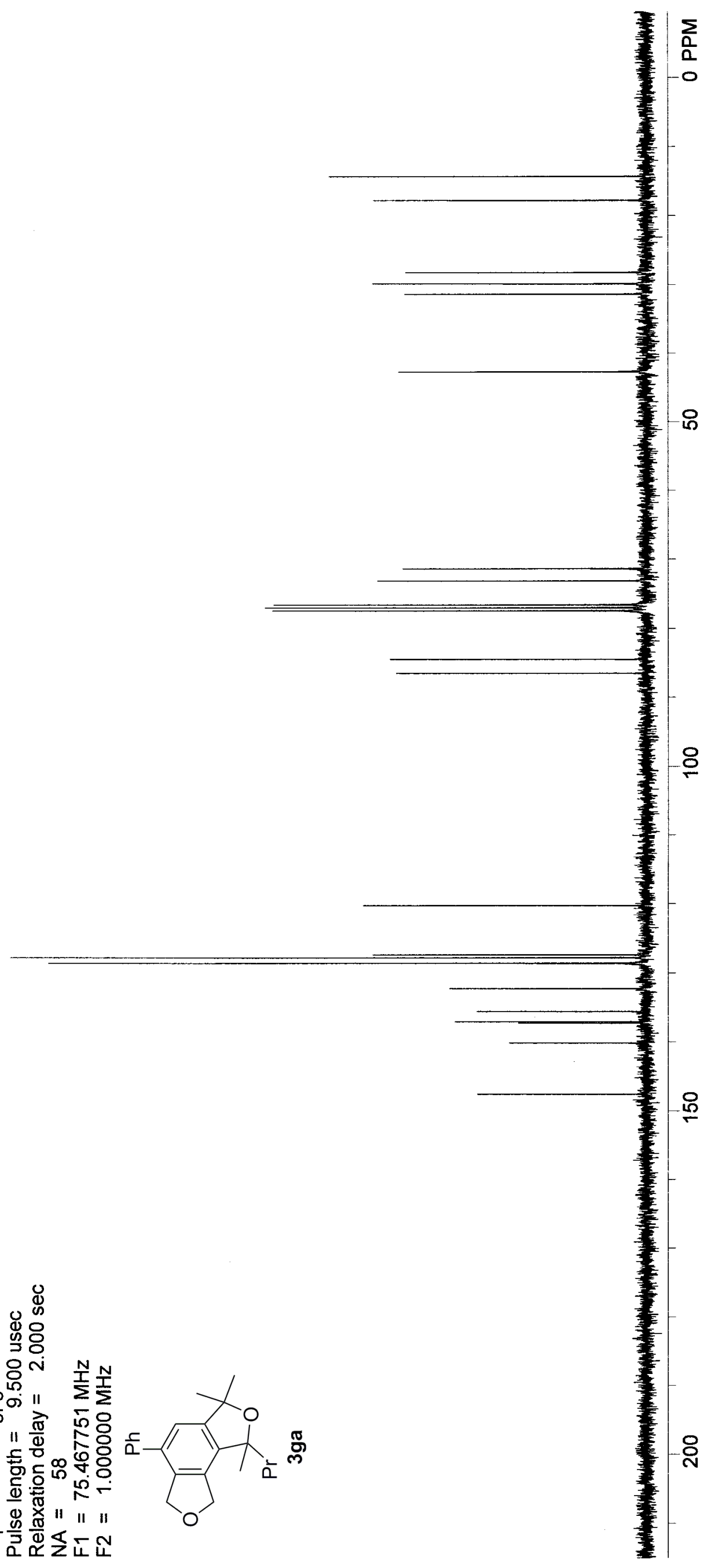



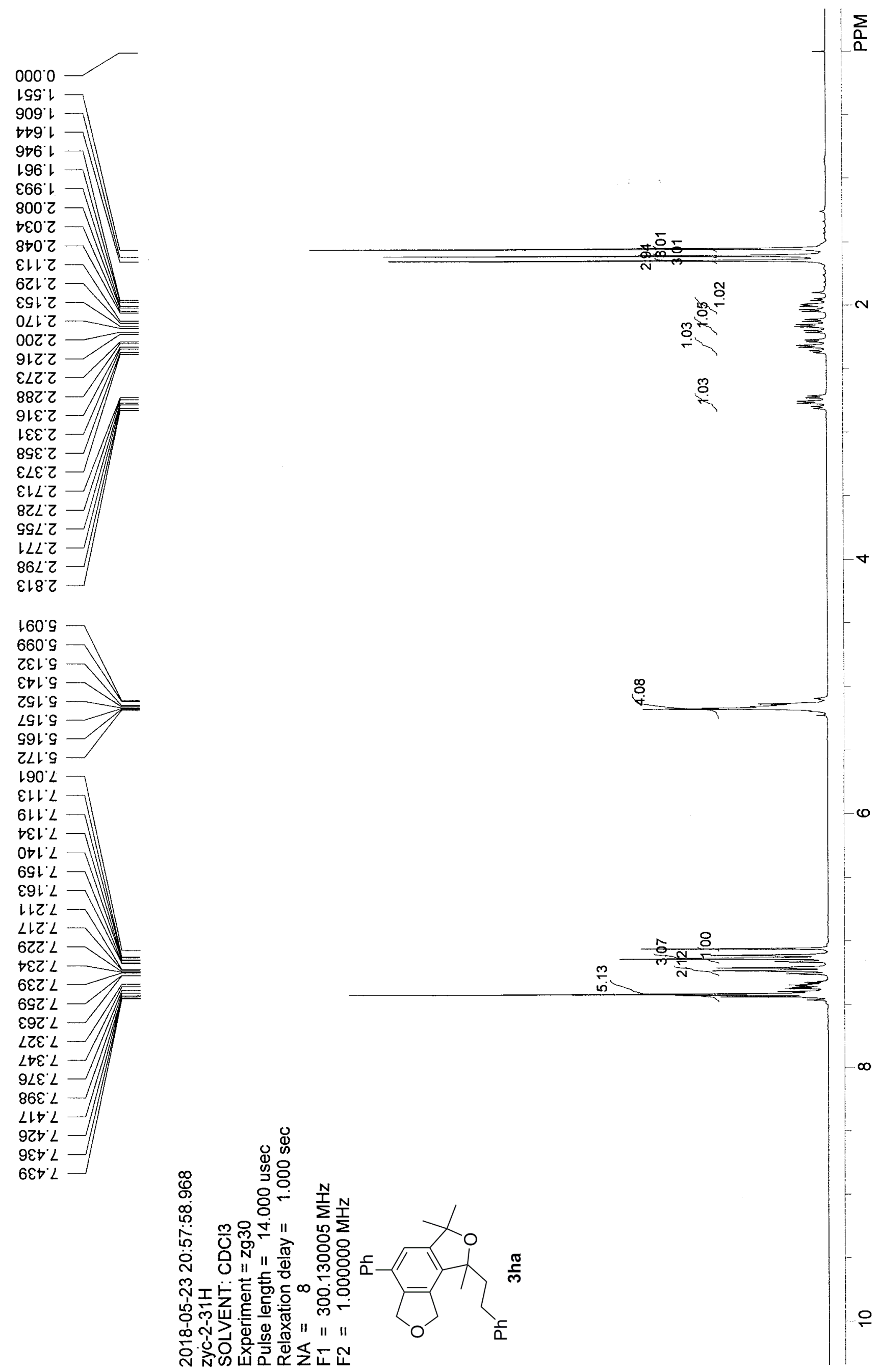
$\angle E Z$ ' $8 Z$

26862

$\varepsilon \varepsilon\llcorner\llcorner\varepsilon$

$9 \varepsilon \sqcup \cdot \varepsilon$

$6 t g^{\prime}$ 乙

$\angle S^{\circ} \varepsilon L$

89G $9 L$

$000 \angle L$

E乙॰ $L L-$

LCL $\triangleright 8$

$\rightarrow 91.98$
$6 \varepsilon \varepsilon^{\prime} 0 Z$

G29'G2

StV $\angle Z I$

†GZ 82

21982

$\angle 0 \varepsilon^{\prime} Z \varepsilon$

$608 \mathrm{~g}$

$609{ }^{\circ} 9$

$\varepsilon \triangleright Z^{\prime} \angle \varepsilon$

8200t

86 '乙2

$8 \varepsilon G^{\circ} \angle \supset L$

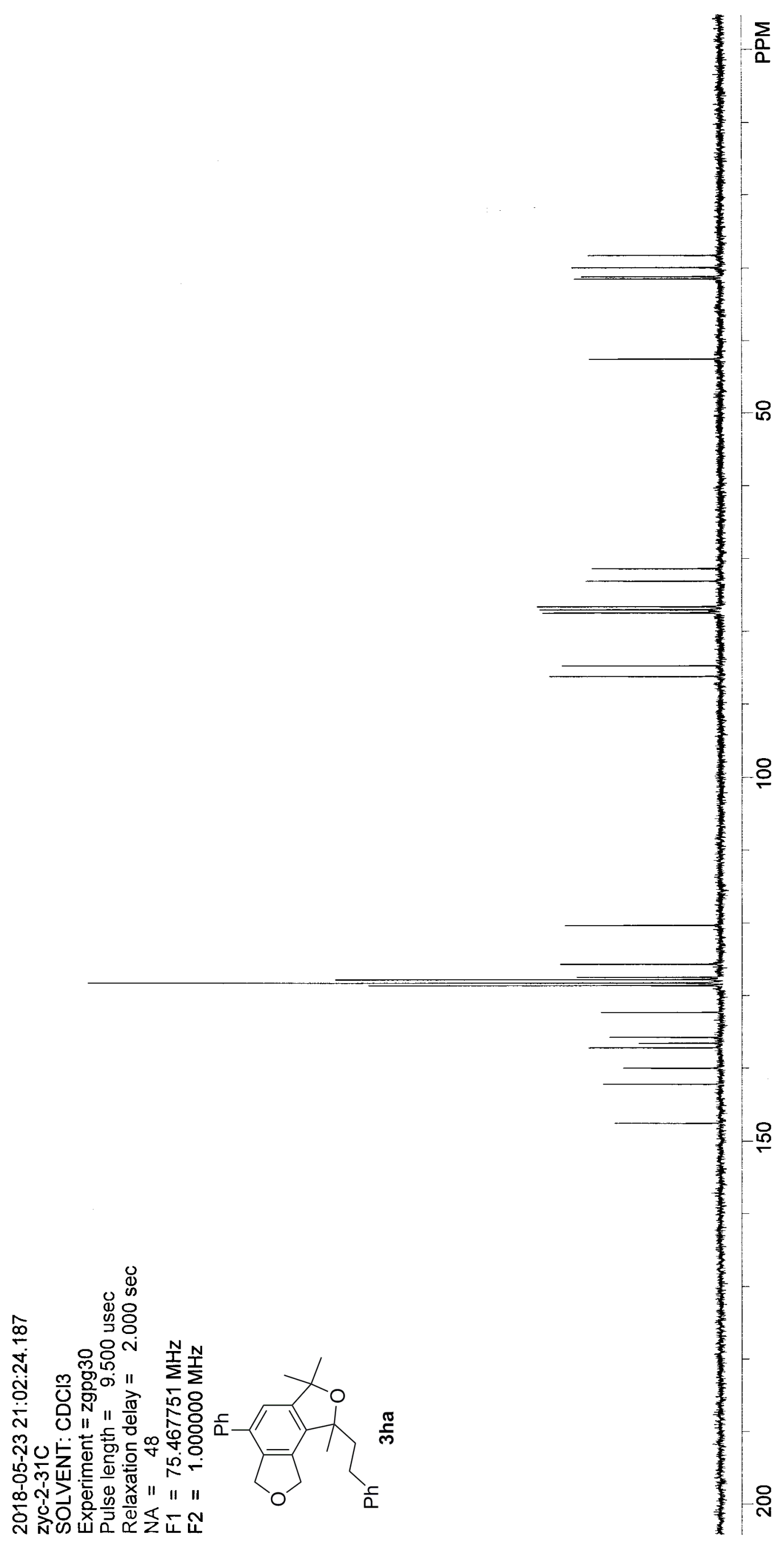


$\varepsilon \leftarrow L$

$0 \succ 8^{\circ}$ L

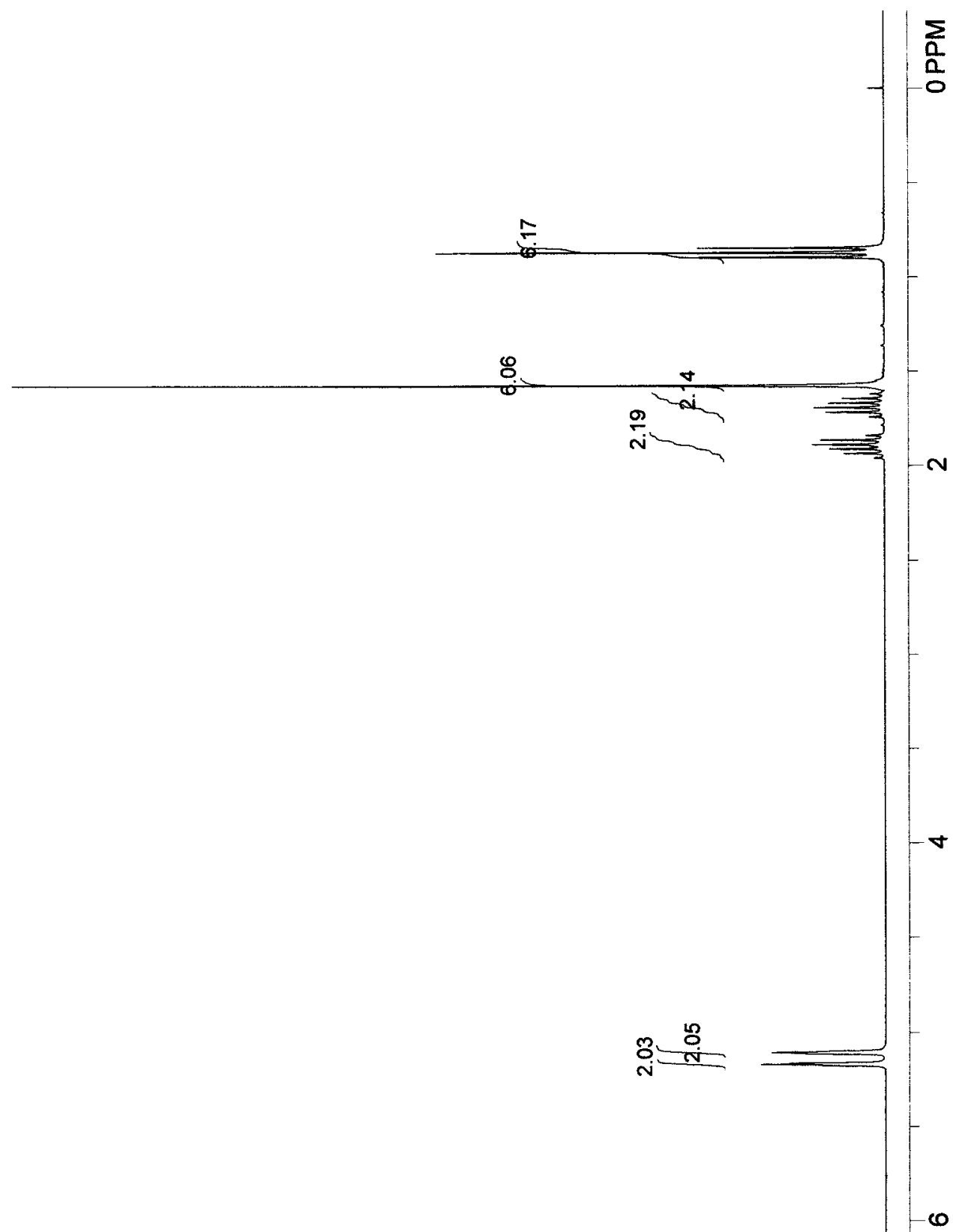

$688^{\prime} \mathrm{L}$

ZL6!

$\angle E 6^{\circ} \mathrm{L}$

296.
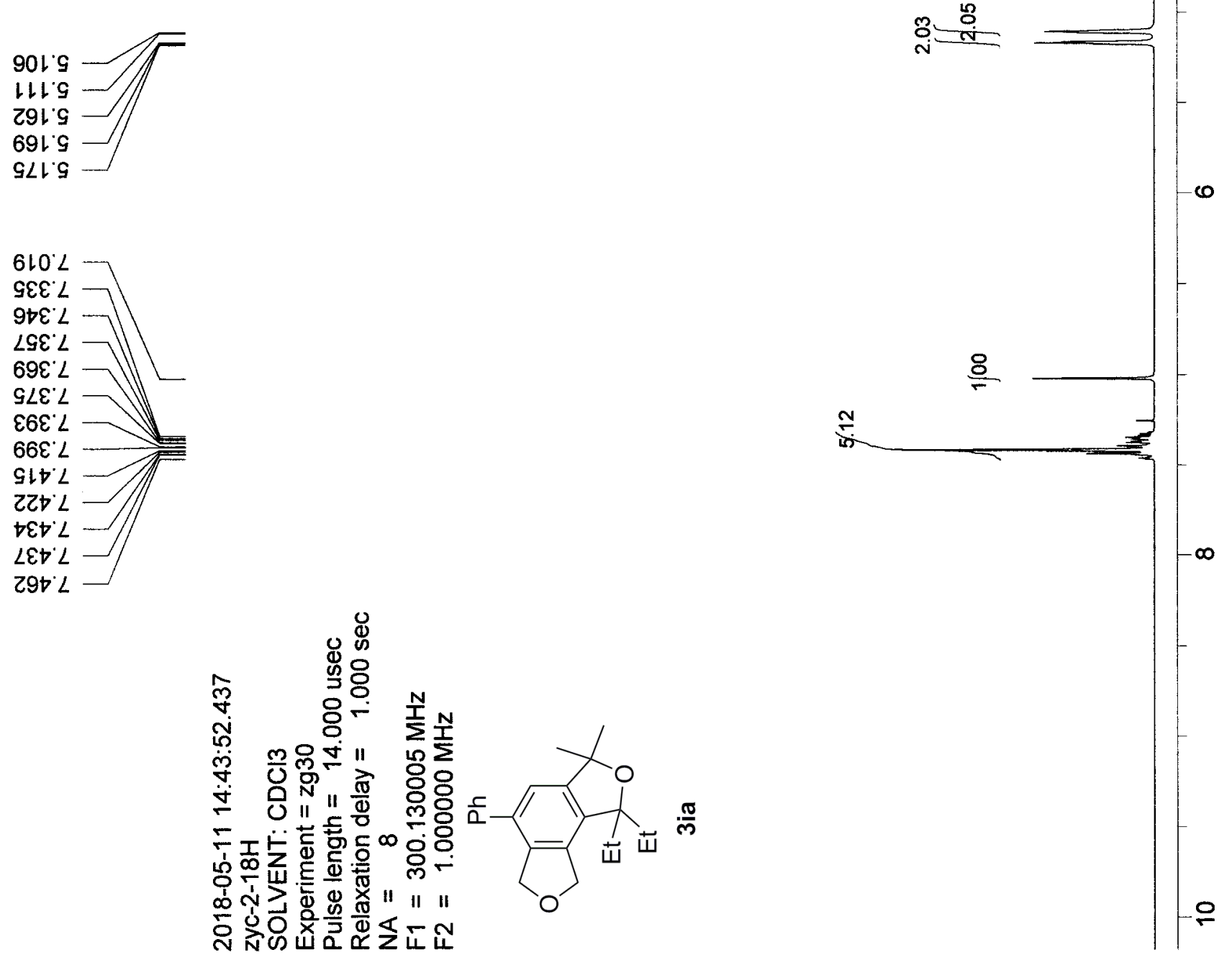
$899^{\circ} L L$
ZレК $E L$

$\angle \angle G^{\circ} 9 \angle$

$000^{\circ} L L$

$\varepsilon 2 \nabla^{\circ} L L$

ZLE' $\triangleright 8$

Z 56.68

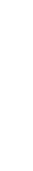

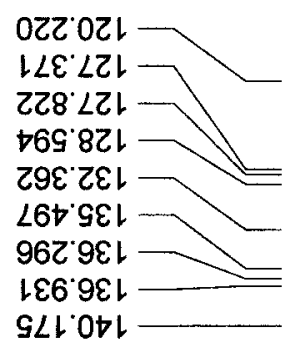

$2008+1$

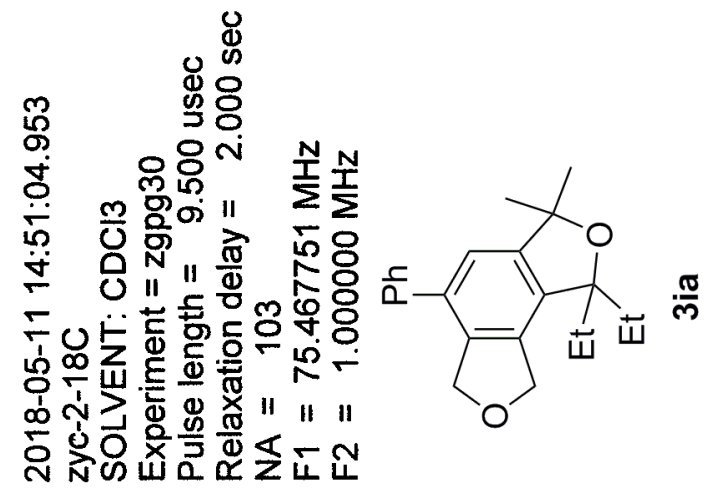




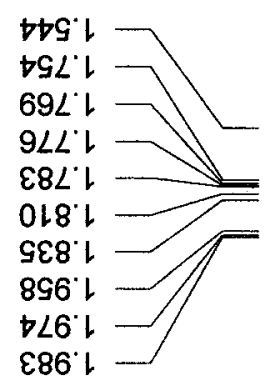

1919

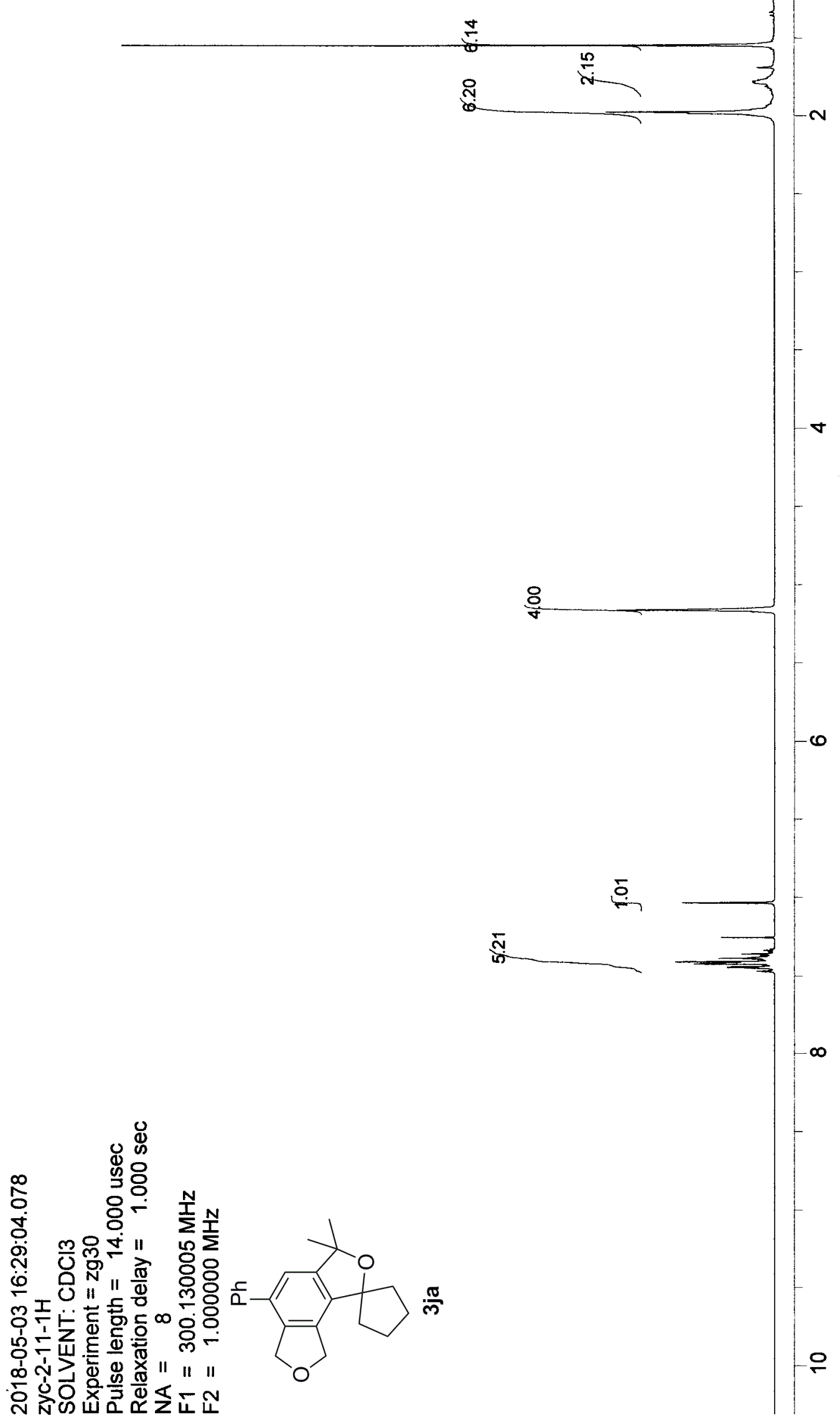

$\varepsilon 86$

\&

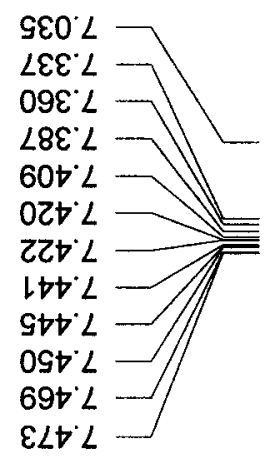




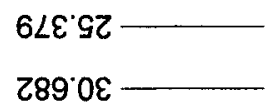

tSSOt

002 LL

$\angle G O{ }^{\circ} \& L$

$\angle L G^{\prime} 9 L$

000LL

$\varepsilon Z \nabla^{\prime} L L$

sttt

06t 86
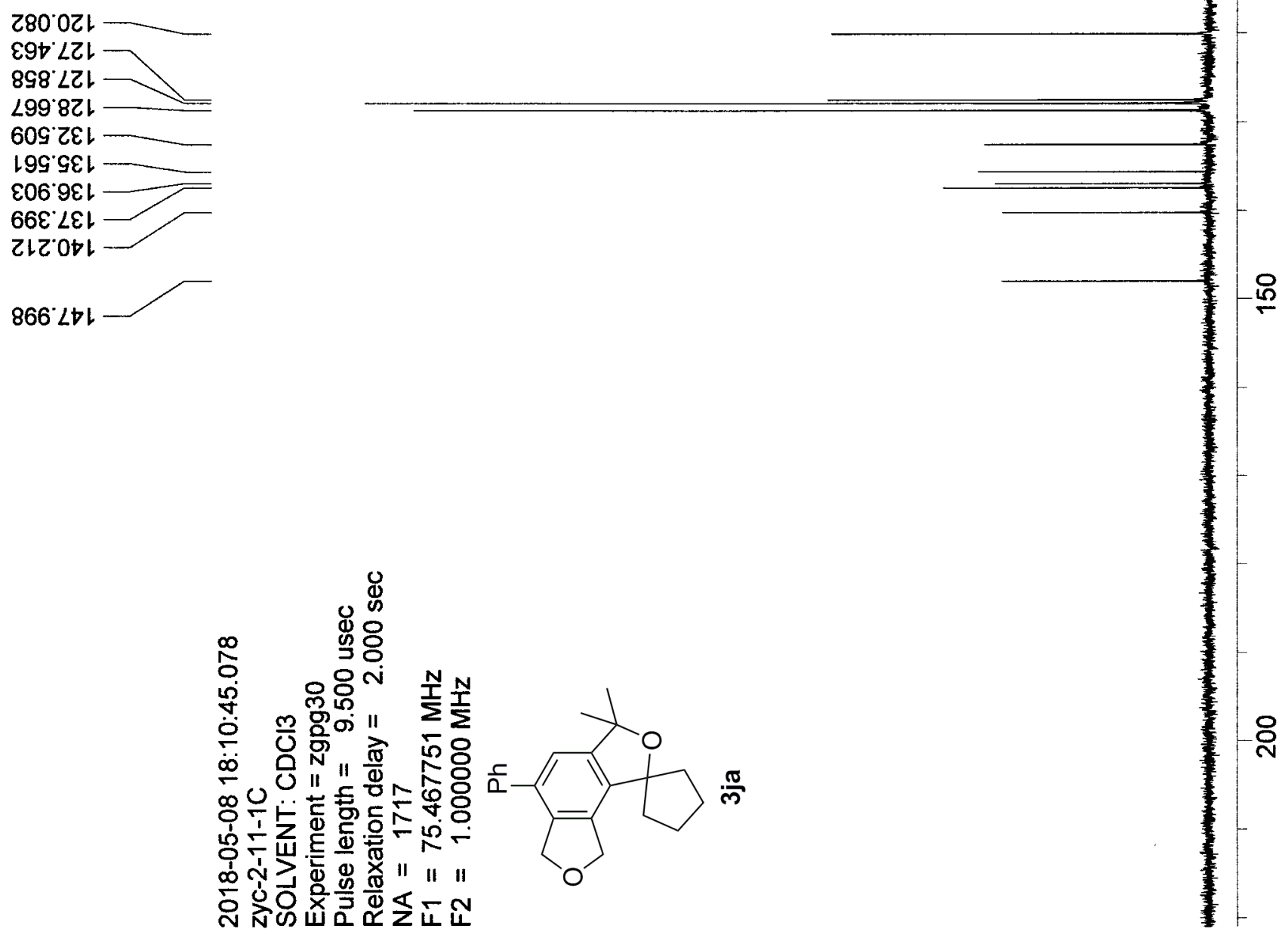

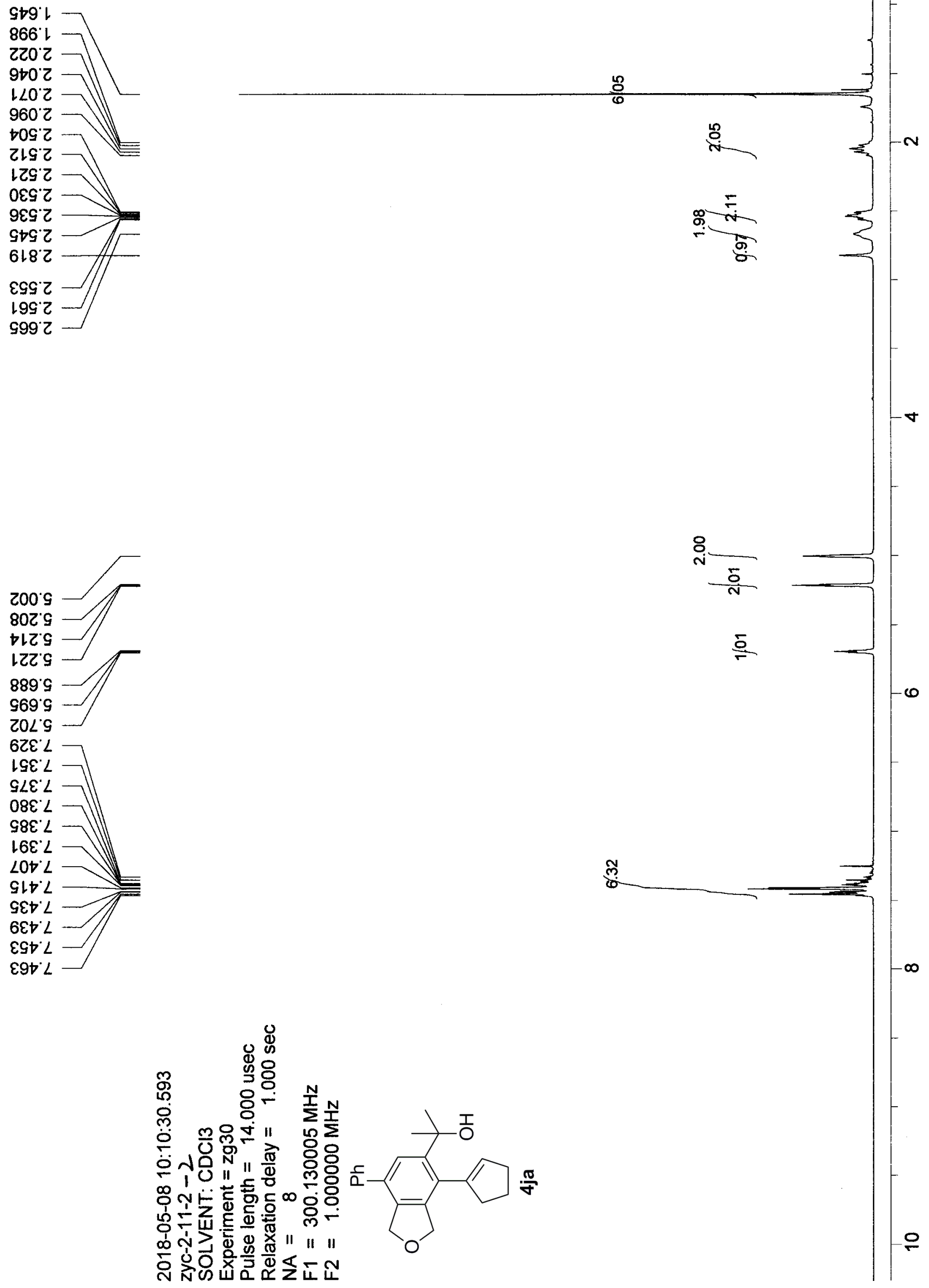
$0+92 \varepsilon$

6乙乙 $\varepsilon \varepsilon$

$0 \angle 8^{\circ} \angle \varepsilon$

$\varepsilon \nleftarrow \nabla^{\prime} \varepsilon L$

$8 \varepsilon 8^{\circ} \varepsilon L$

$9 Z \nabla^{\circ}+L$

$\angle \angle G^{\prime} 9 L$

$000 \angle L$

$\varepsilon Z \nabla: L L$

\&9L'GZL

$\angle \downarrow \nabla Z L$

$99 \angle \angle Z L$

899'8ZL

969'6Zl

七8 621

$\angle \varepsilon Z \triangleright \varepsilon L$

6Lo'GE

\&L6'6\&l

$\varepsilon 80^{\circ} 0$ t

8†ย๋ヤเ

$\varepsilon 6 L ' 9 \downarrow L$

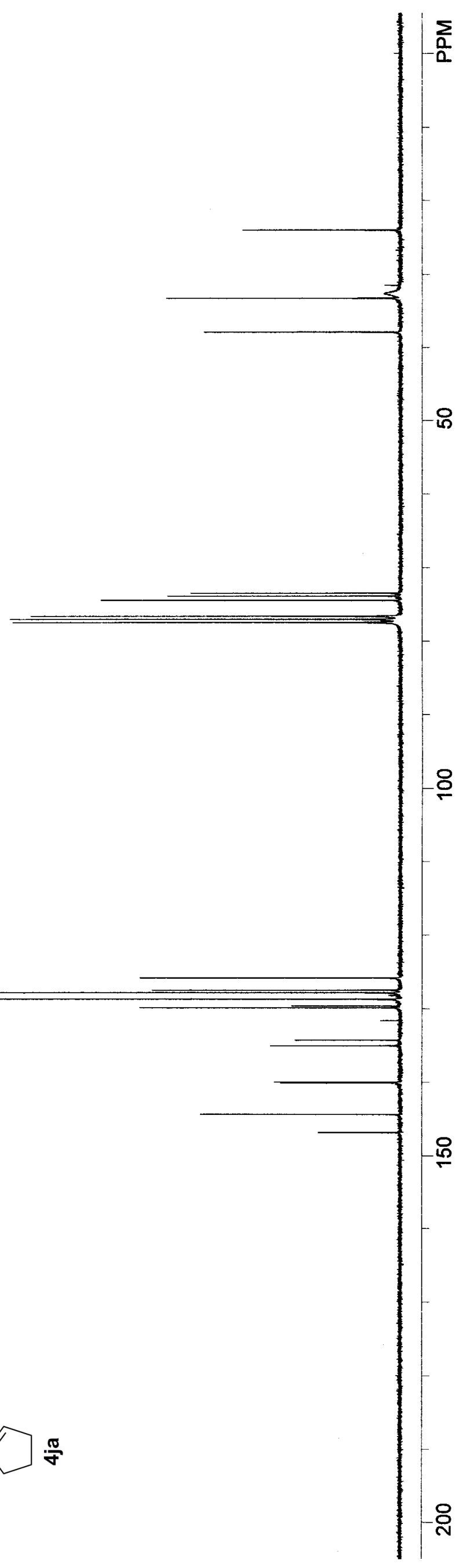



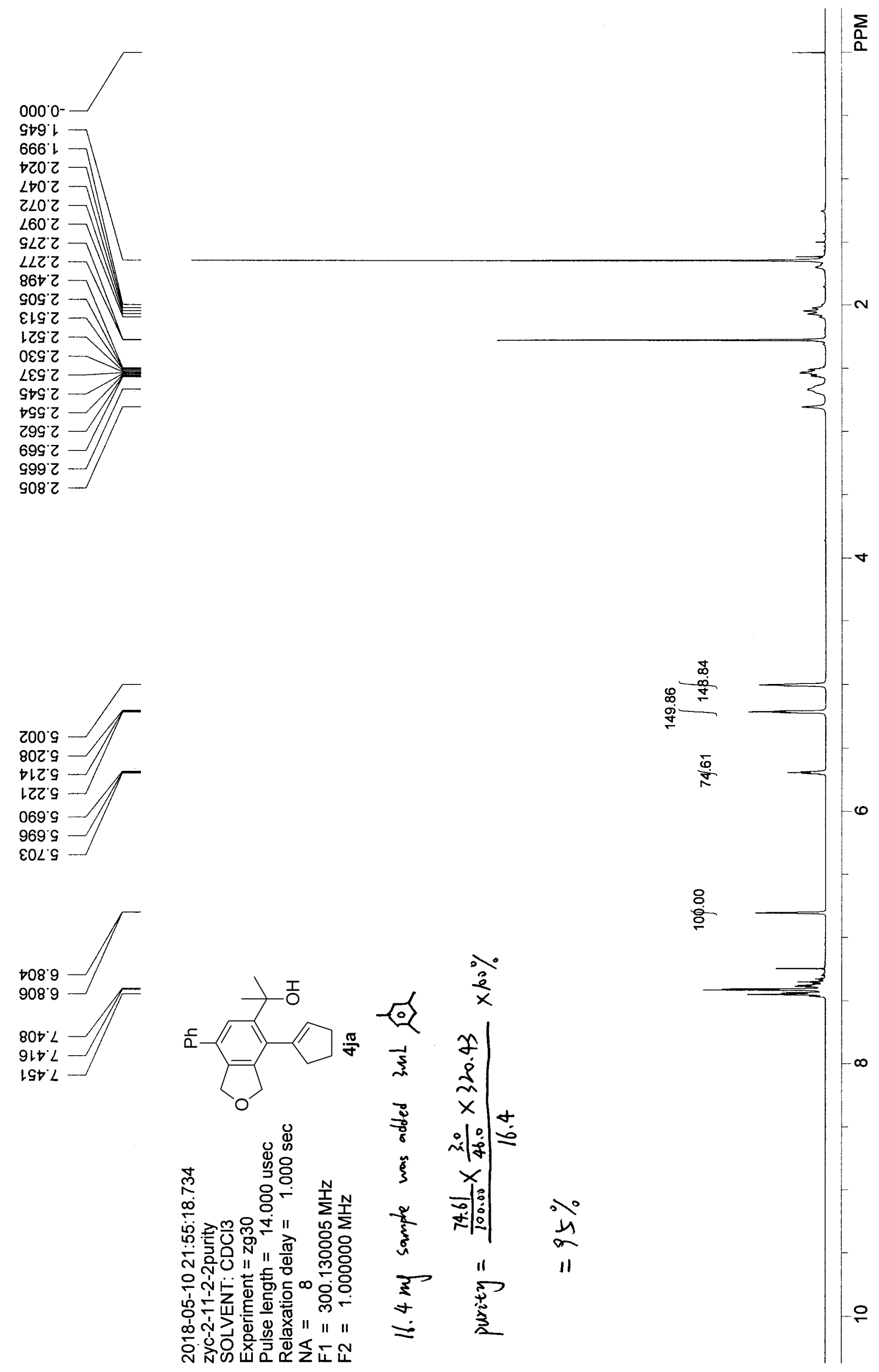

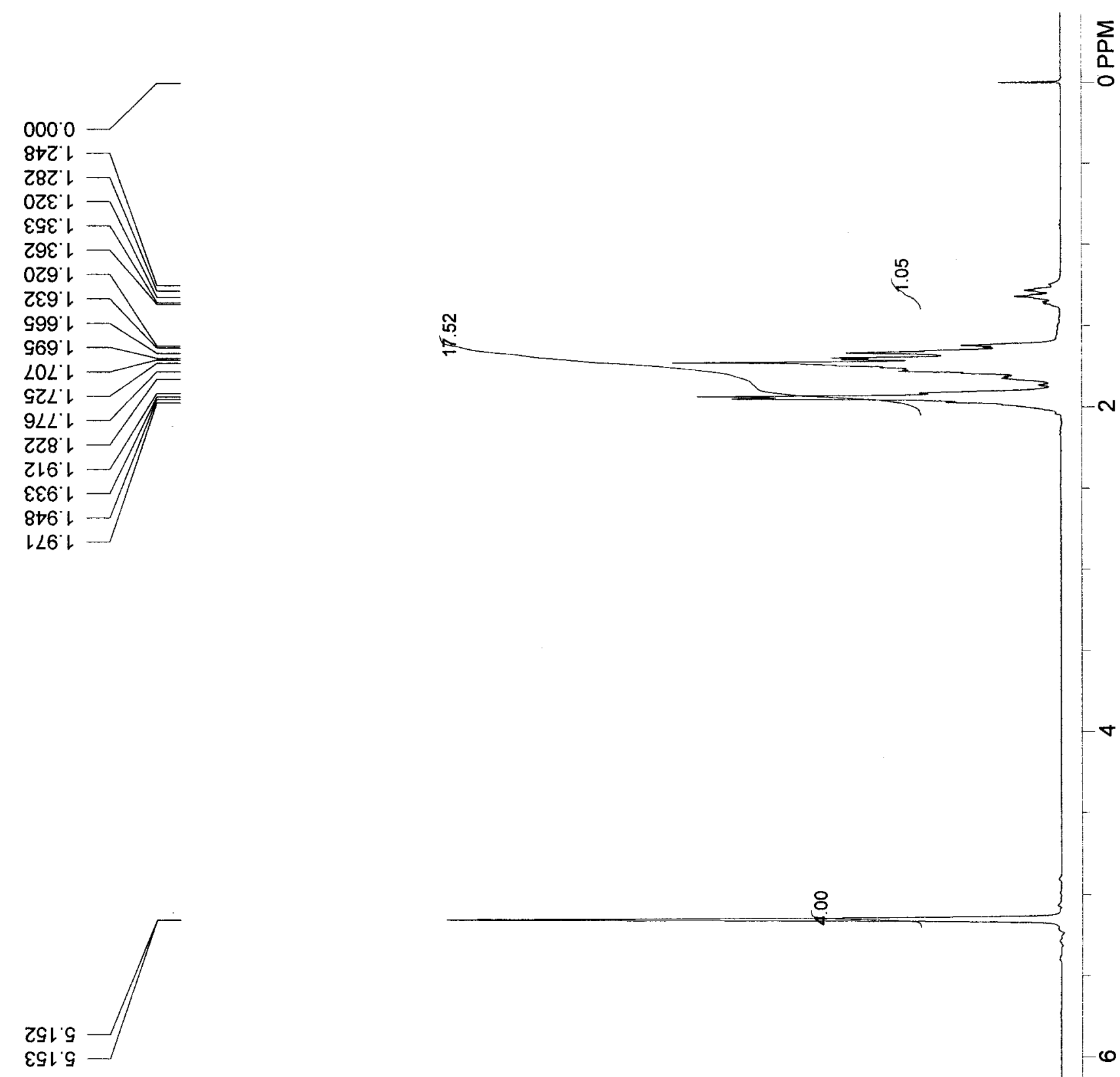

8
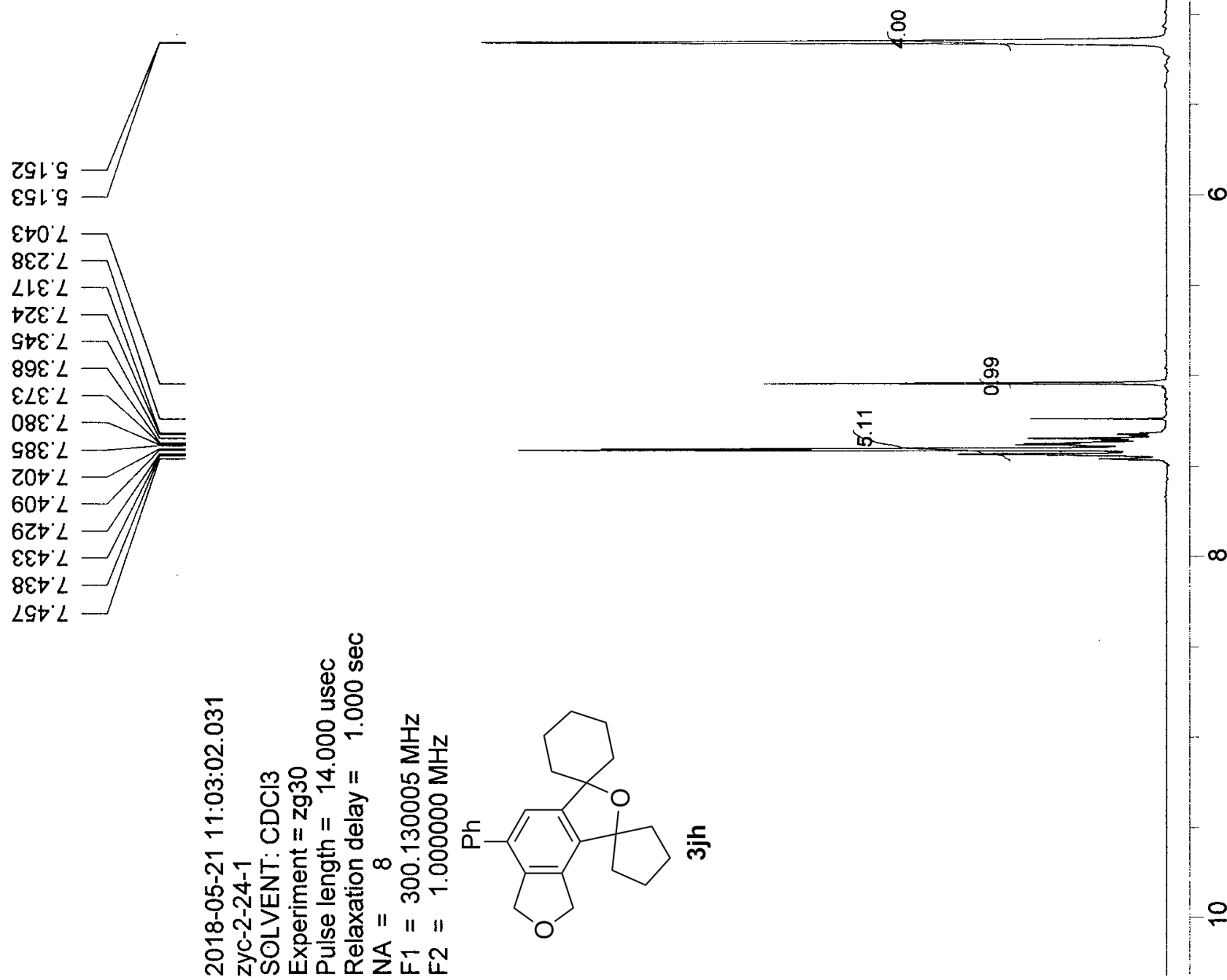
18198

$\$ 98 \gtrsim 6$

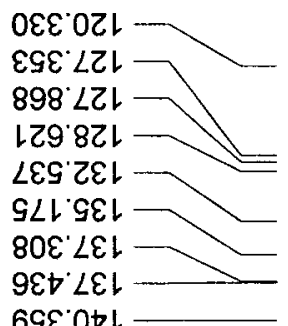

$69 \varepsilon^{\circ} 0 \triangleright t$

$\rightarrow \angle \varepsilon^{\circ} 8 D$

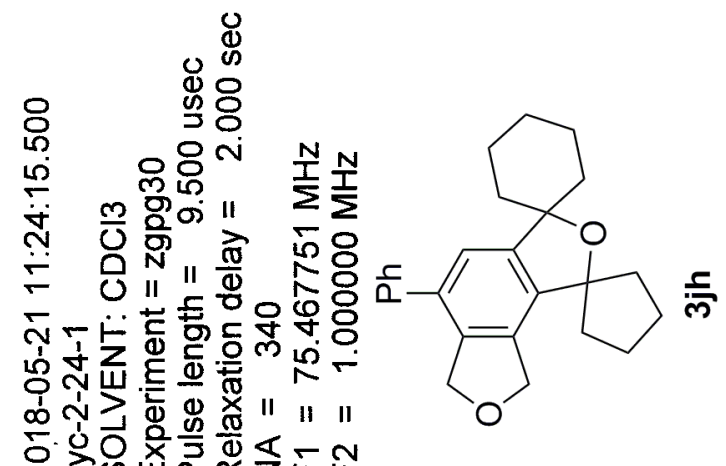

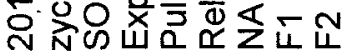


006 '

El6!

七6 ᄂ

996 .

$\angle 20 Z$

$\mathrm{ZSO} \mathrm{Z}$

$9 \angle 02$

$209 \mathrm{Z}$

6092

E\&S 2

IS9'

8992

$\checkmark \angle 9^{\circ} Z$
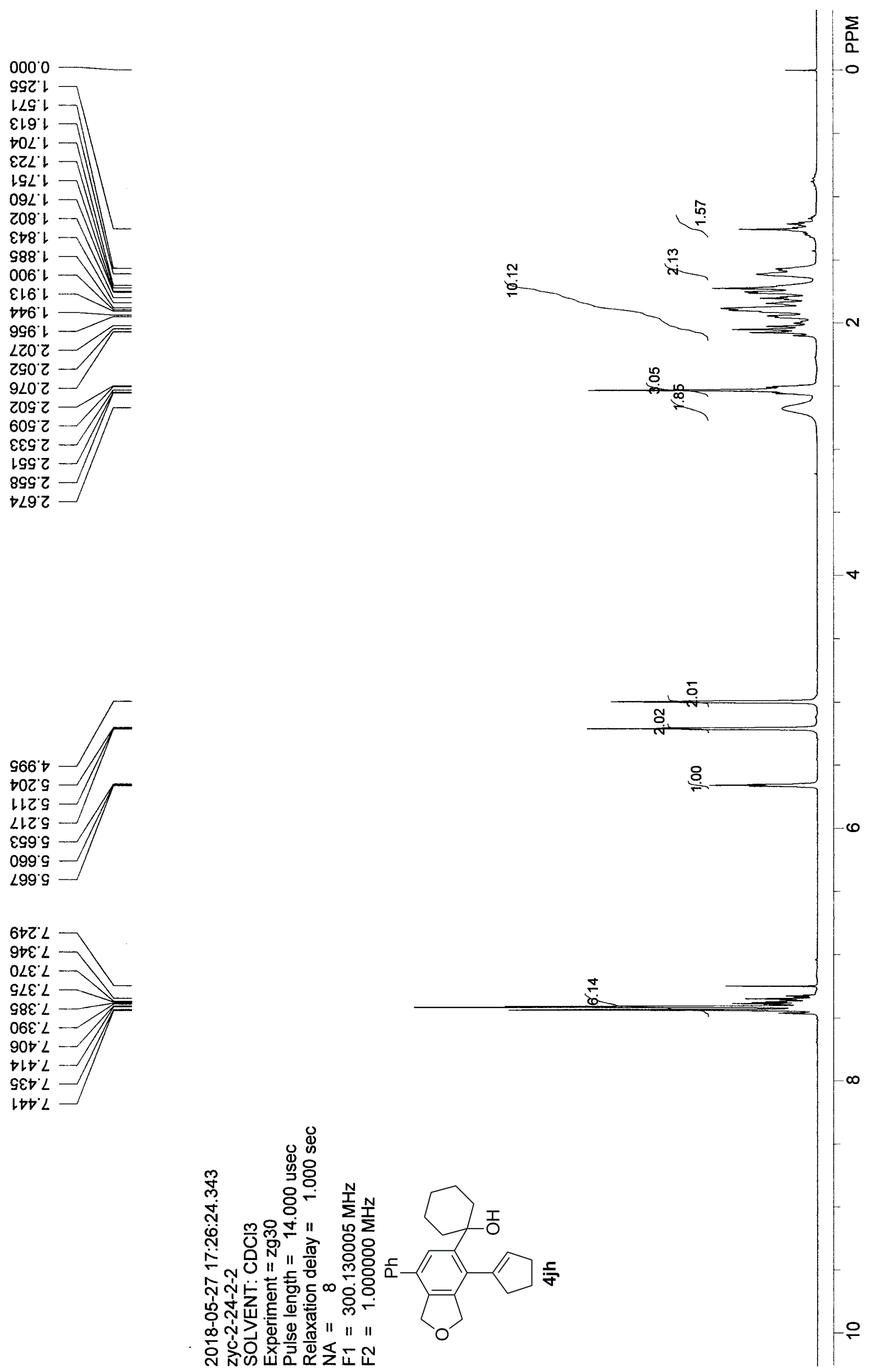

8

$6+Z L$

$9 \triangleright \varepsilon \angle$

$0 \angle E^{\circ} \angle$

$S L E L$

S8E $L$

$06 \varepsilon \angle$

$90 \sigma^{\circ} L$

tLt L

SE⿱ $L$

$100<$ 
$6 乙 2 \varepsilon \varepsilon$

$00 L^{\prime} 8 \varepsilon$

9६8 $8 \varepsilon$

ISZ Ot

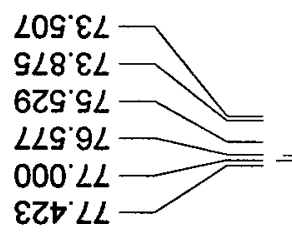

996 $9 Z$

$66 \varepsilon \angle Z$

G8L $\angle Z L$

899.8Z

L9E'6ZL

l $\angle 862$ L

OL乙'†ยL

668'十हL

$286.6 \varepsilon \mathrm{L}$

s8. 0 เ

$\angle \varepsilon 6$ เ

$06 Z \angle \vdash L$

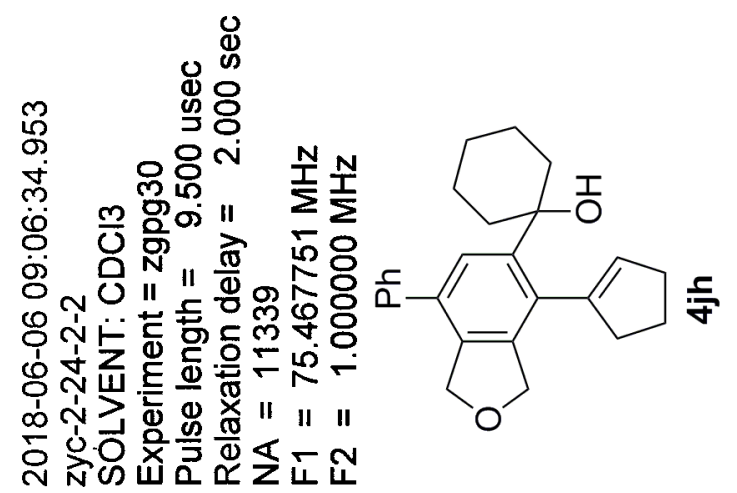

$-8$ 
$\left\{\begin{array}{l}\sum \\ \frac{z}{0} \\ -\frac{2}{0}\end{array}\right.$

SGZ

699'

L19.

$0 S \angle L$

2 8 . 1

ع88 $\mathrm{L}$

$920 \mathrm{Z}$

$6+0$ 己

$\checkmark \angle O^{\circ} Z$

$1 \angle 2 \cdot 2$

LEG'

$089 \mathrm{Z}$

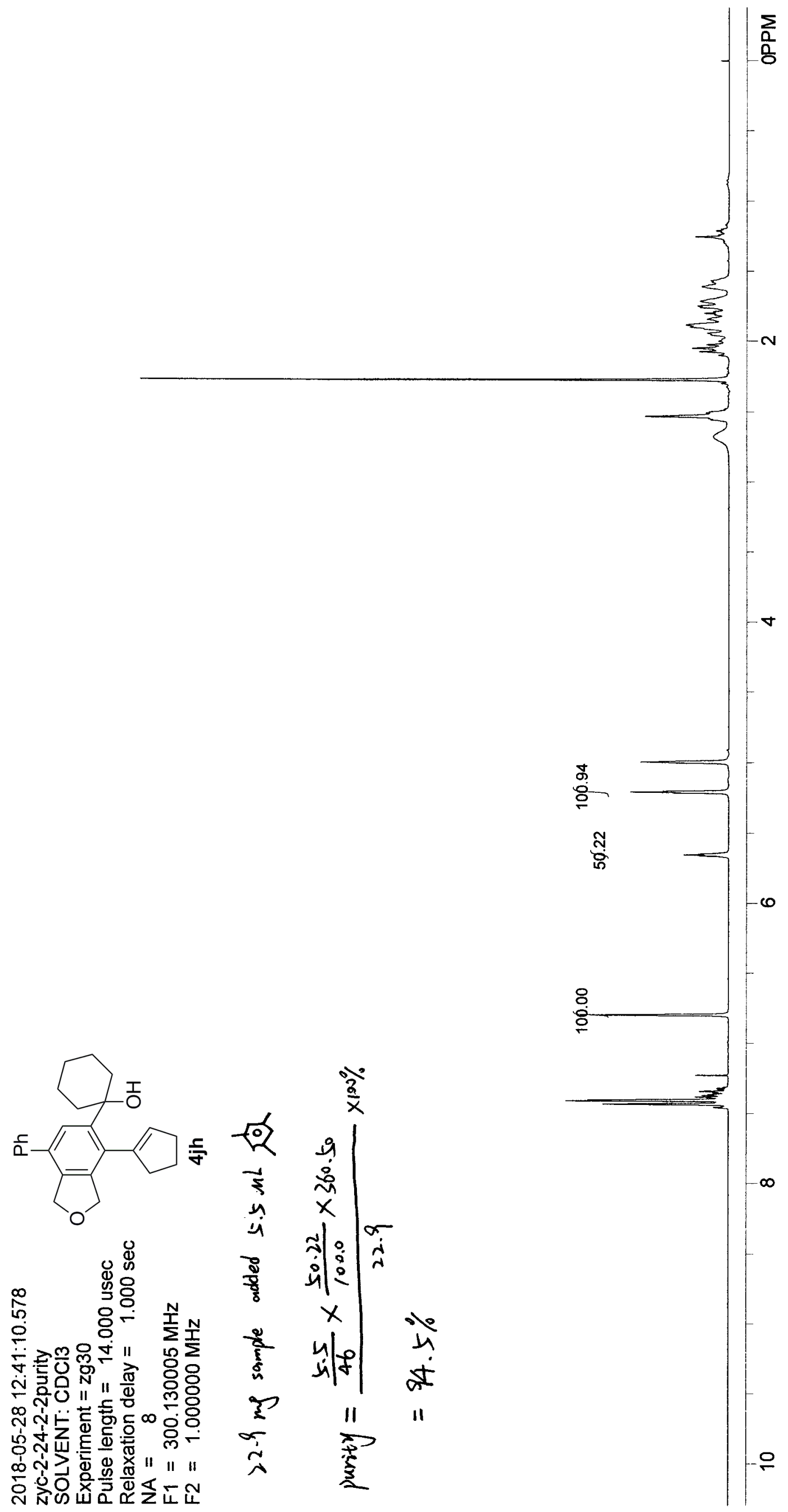

Z0Z'

$802 \mathrm{C}$

SLCS

199'

$\angle 99^{\circ} 9^{\circ}$
$\square 99^{\circ} 9^{\circ}$

$008 \cdot 9$

1089

LZE L

$\varepsilon \sqcup \varepsilon\llcorner$

ZLE'L

tOT 2

IttL

Eहt $L$
9Gt $L$

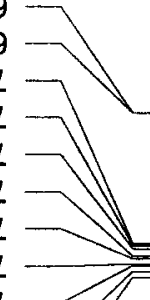

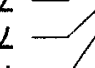



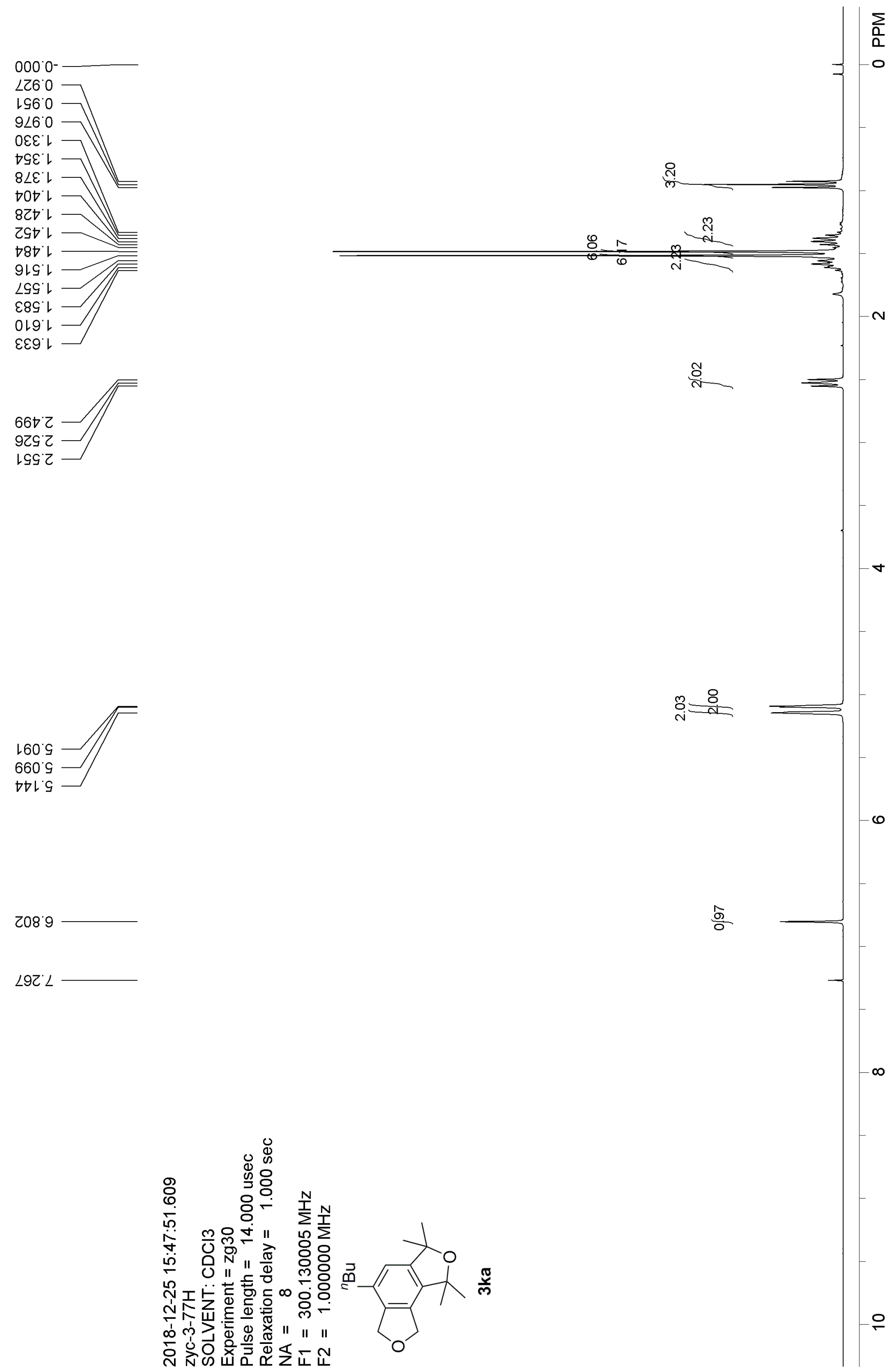


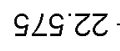

เ8เ乙E

$\nabla 9 L^{\circ} \varepsilon \varepsilon$

$\angle 9 T^{\circ} L$

ㄴIG'CL

$\angle \angle S^{\circ} 9 \angle$

$000 \angle L$

EZt LL

$\downarrow ९ \mathcal{C}^{\prime} \varepsilon 8$

$9 \nabla S^{\circ} \triangleright 8$

$6 L L 6 L$

$\nabla 乙 \varepsilon\llcorner\varepsilon\llcorner$

$008 . \varsigma \varepsilon$

$\downarrow L L 9 \varepsilon L$

$8 \varepsilon 9 \angle \varepsilon L$

OL9.9t

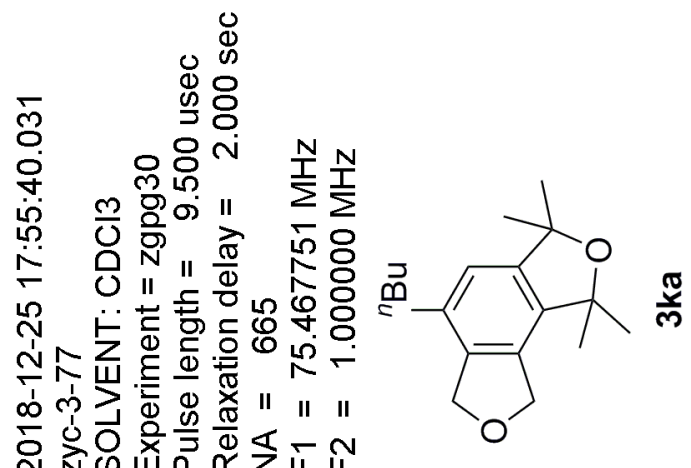



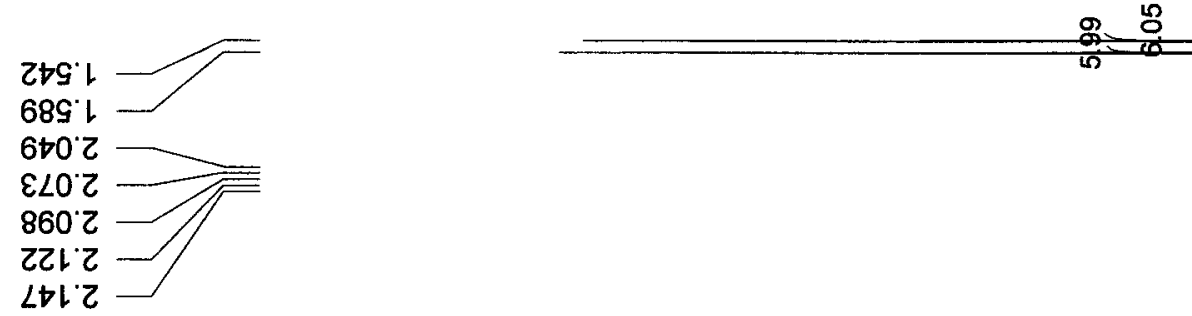

8162

\&†6 2

$0<6^{\circ} 2$

966 乙
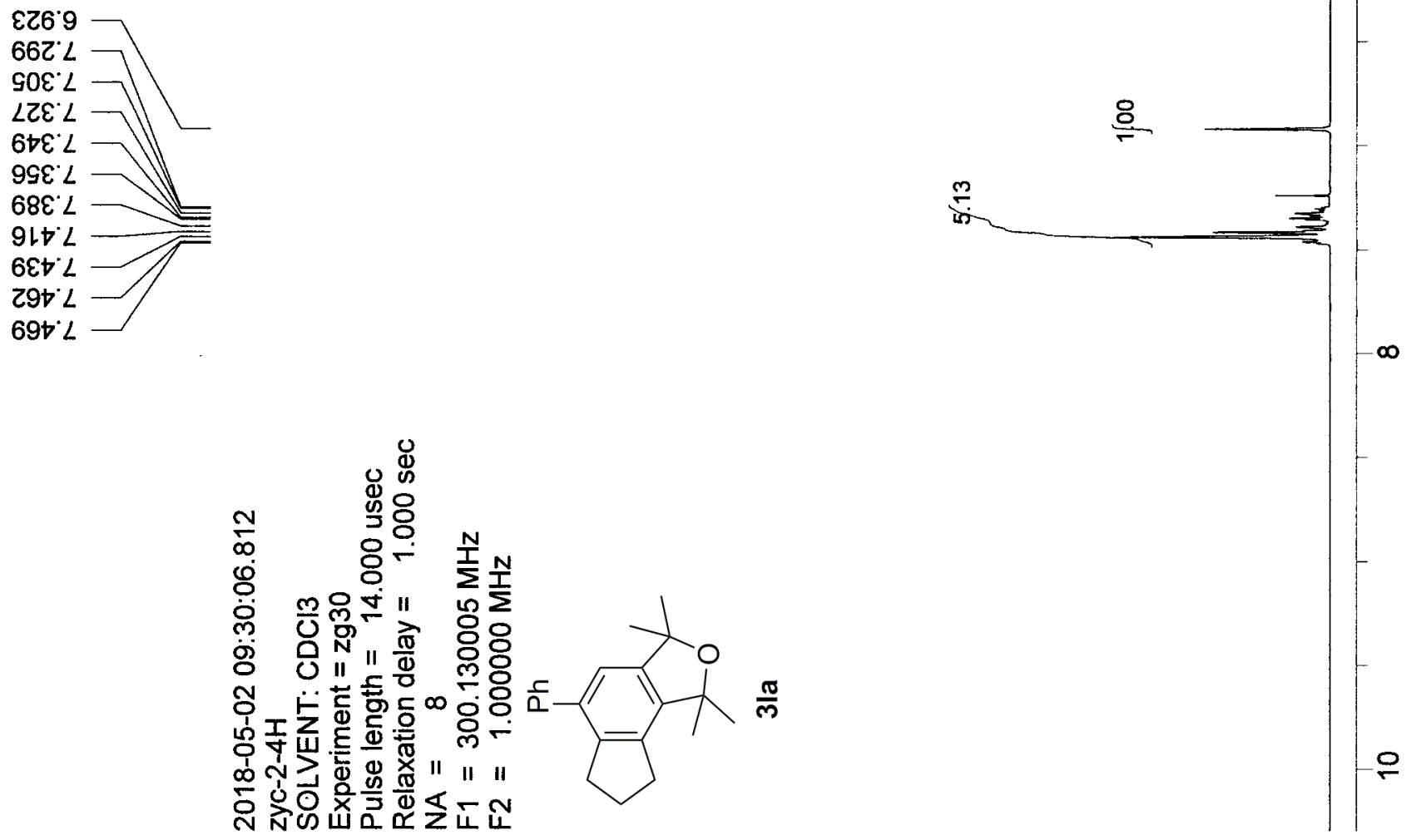
LLG' $9 L$

$000^{\circ} \angle$

$\varepsilon Z \downarrow \angle L$

$1+0 \div 8$

$\varepsilon \varepsilon \vdash \triangleright 8$ 20t 6 L

628 92

86 ' $^{\prime} \mathrm{Cl}$

GLG' $8 Z$ L

SOL $\angle E L$

$698^{\circ} \angle E L$

OSE 0 '

ELGLا

6Lレて๖レ

EtG 9 b

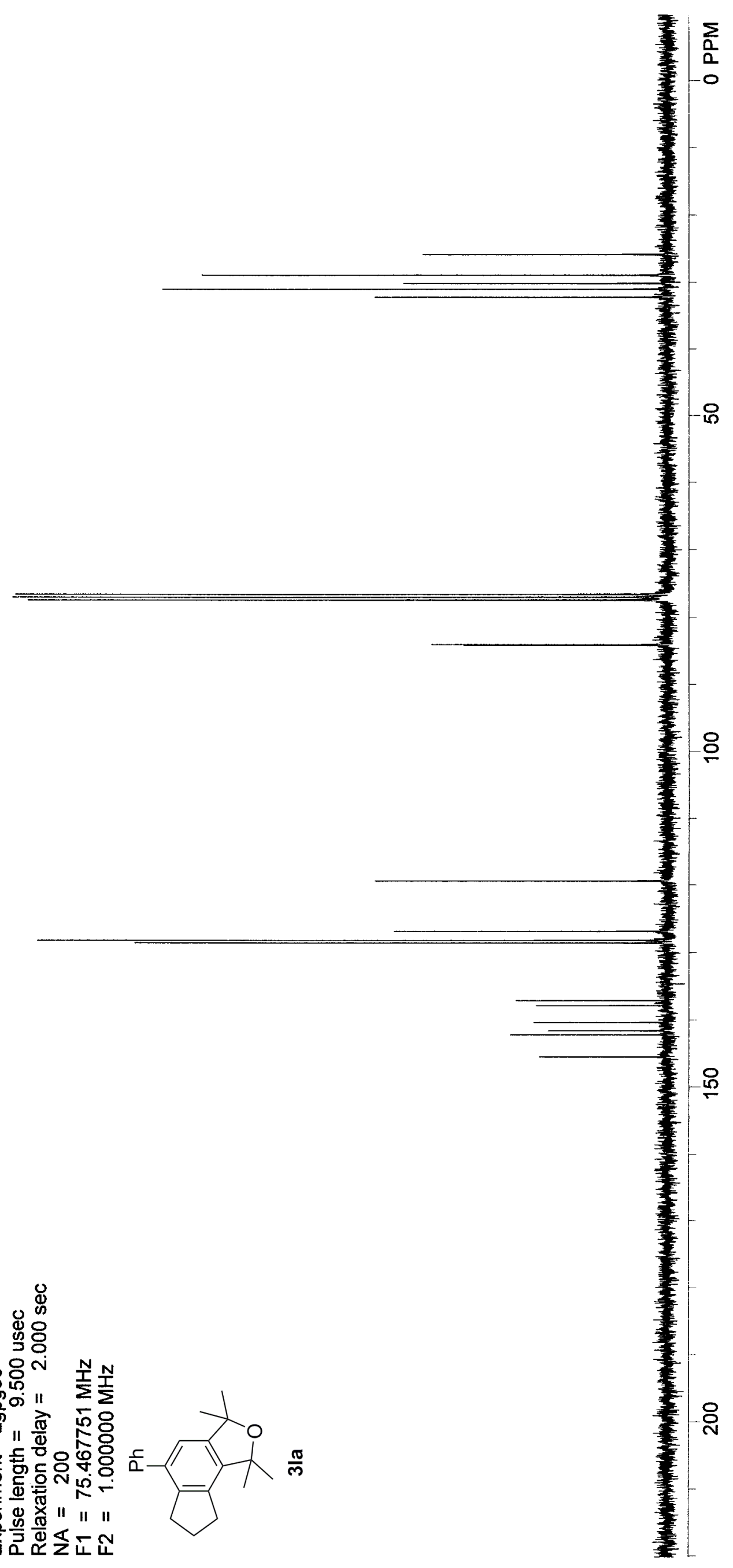



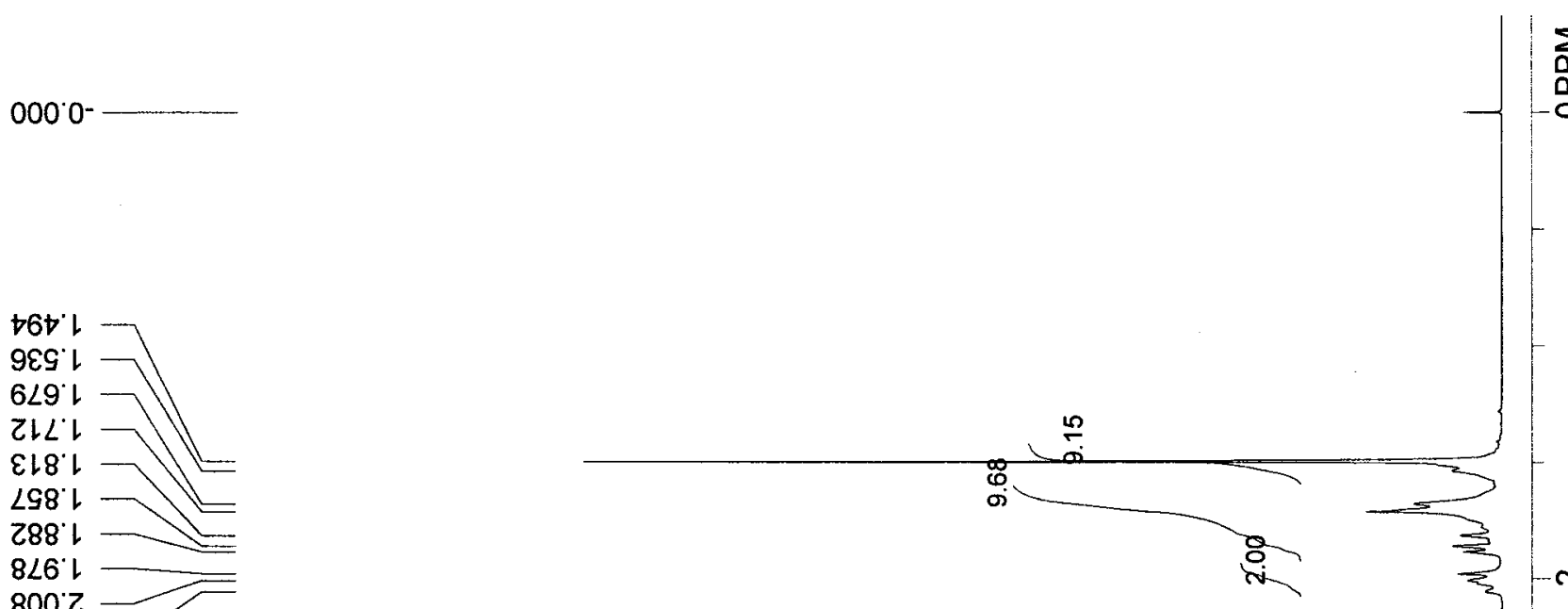

ชั

ZOE: $L$

†० $\varepsilon^{\circ} L$

$60 \varepsilon^{\circ} L$

IE $\varepsilon^{\circ} L$

$9 \varepsilon \varepsilon^{\circ} L$

8Zt L

ZSt $L$

$\varepsilon \varepsilon L L$

$09 L L$ 


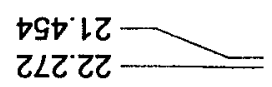

$826^{\circ} \textrm{CZ}$

$\angle \forall Z^{\prime} 8 Z$

000.62

$98 \nabla^{\circ} 8 \varepsilon$

Z8E. IS

$\angle 26{ }^{\circ} Z 9$

$\angle \angle 9^{\circ} 9 \angle$

$000 \div$

$\varepsilon Z \downarrow L L$

เ৮乙'ع8

เع6.88

†ย况

$\angle E G^{\circ} \angle Z$

$\angle \nabla 9^{\circ} \angle Z L$

I90 82

GLE $6 Z$

ง\&8 $6 \mathrm{Z}$

ง9t $\varepsilon \varepsilon\llcorner$

เ6L $\nabla \varepsilon L$

$6 L \angle 9 \varepsilon L$

$\nabla Z 9^{\circ} 6 \varepsilon L$

$1 \angle L 6 \varepsilon$

乙๖LEヤ

G20 8†

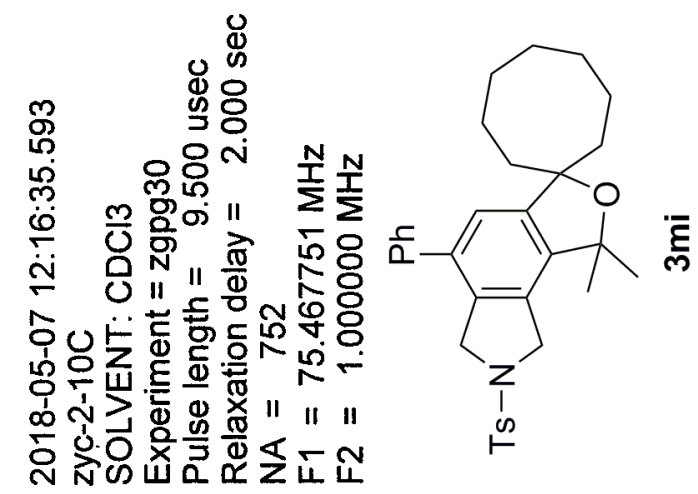


$889^{\circ} 0$

ZLLO

OLZ'

6lz'

$82 Z^{\circ}$

$\angle E Z Z^{\prime}$

†9Z' ᄂ

8LG'।

IZS'

SOL'

$\triangle 2 L '$

$28 L^{\circ} \mathrm{L}$

$068^{\circ} \mathrm{L}$

$\angle 16$.

$696^{\circ} \mathrm{L}$

$100^{\circ} \mathrm{Z}$

$\angle 80^{\circ} \mathrm{Z}$

$960^{\circ} \mathrm{Z}$

6SIZ -

ISL'
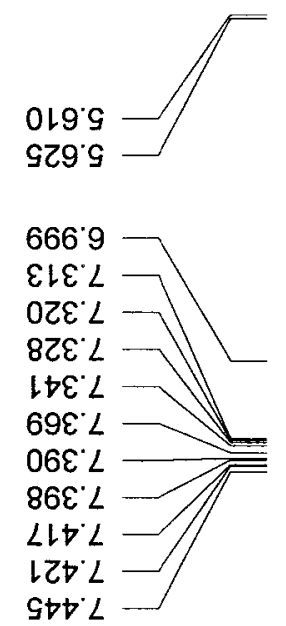

$-1 \mid$

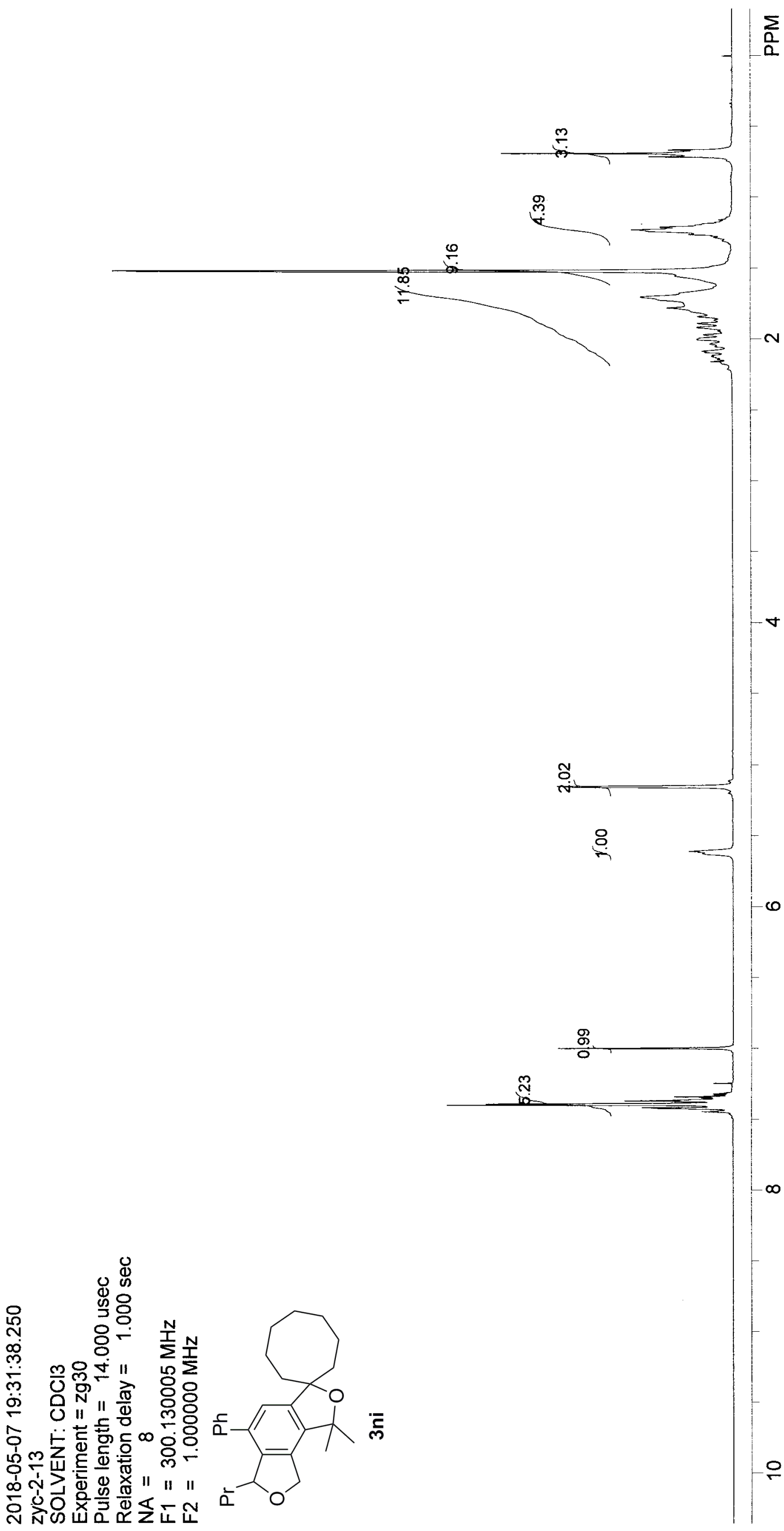




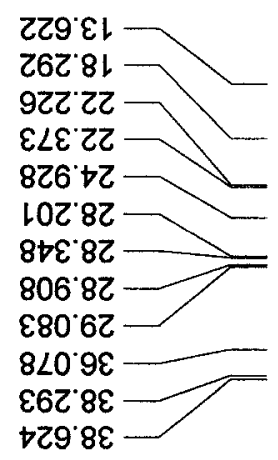

0†8' 69

$\angle \angle G 9 L$

$000^{\circ} \angle L$

$\varepsilon Z \downarrow \angle L$

†๐ ' 8

งเE $\varepsilon 8$

90168

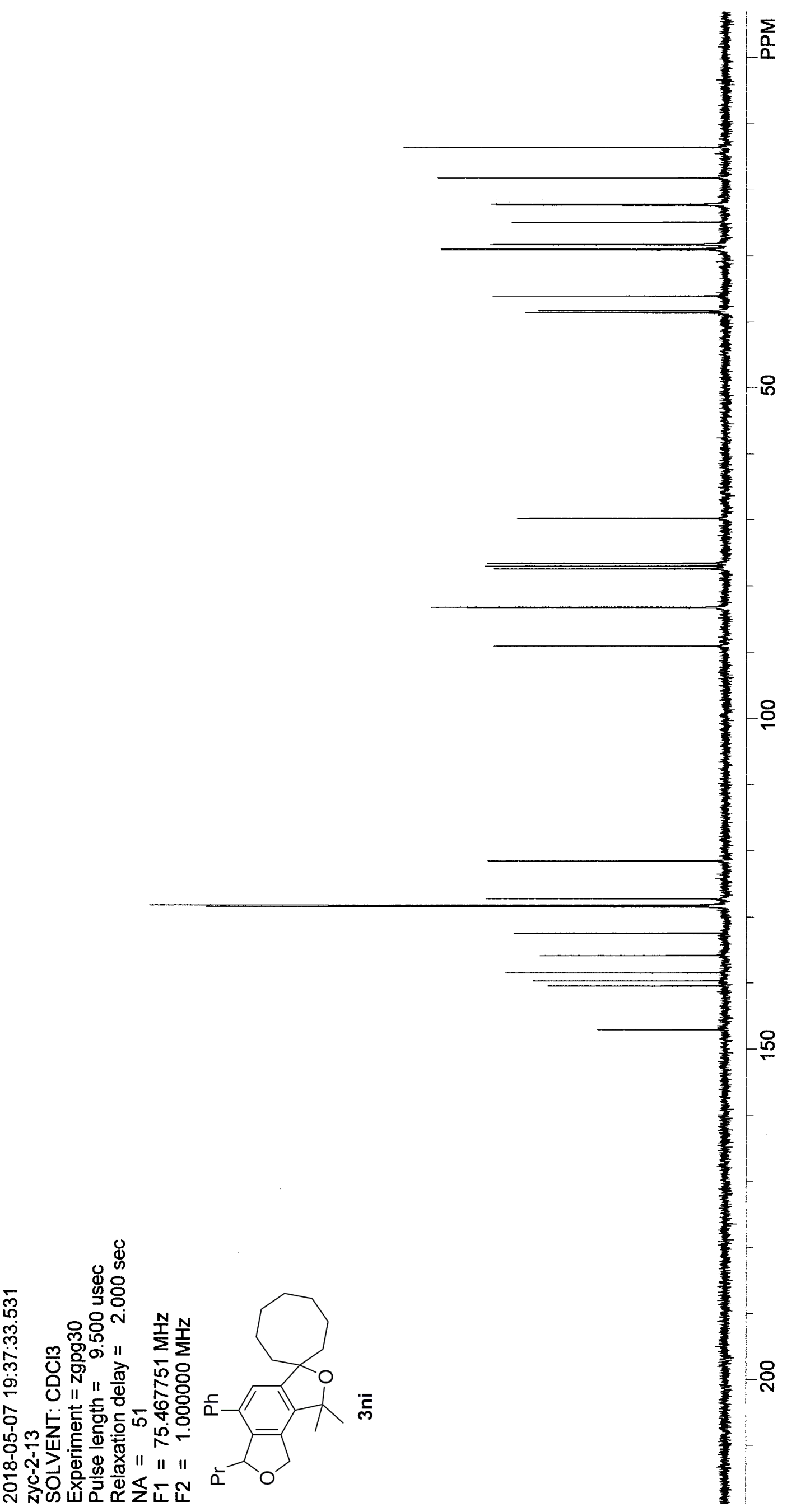

จ๑'に

$\angle 8 L^{\circ} \angle Z$

$6818 \mathrm{C}$

0ᄂt'8ZL

80t ZEL

LE8'GE

† $78 \varepsilon$

$889^{\circ} 6 \mathrm{EL}$

09t OtL

เレL๖L 


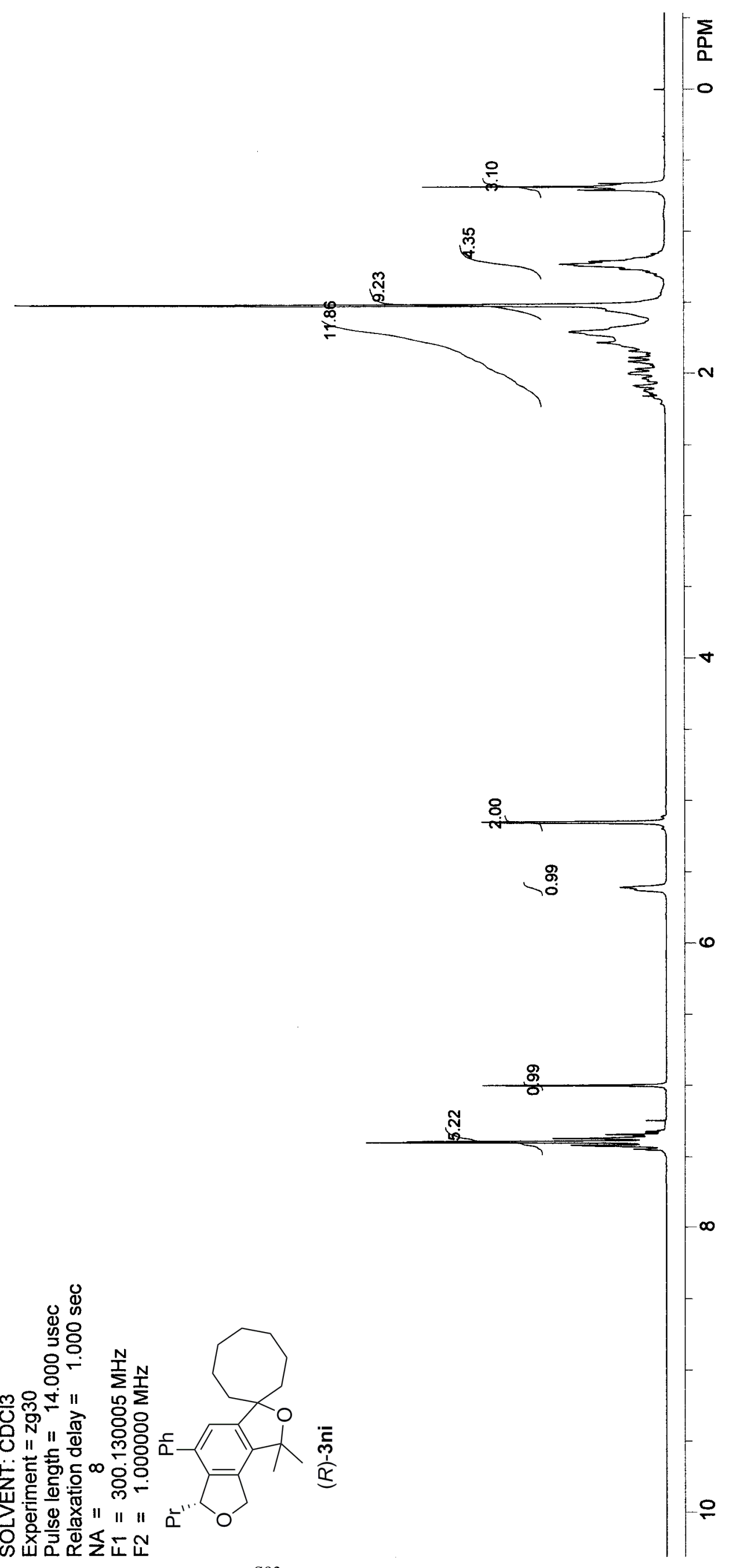



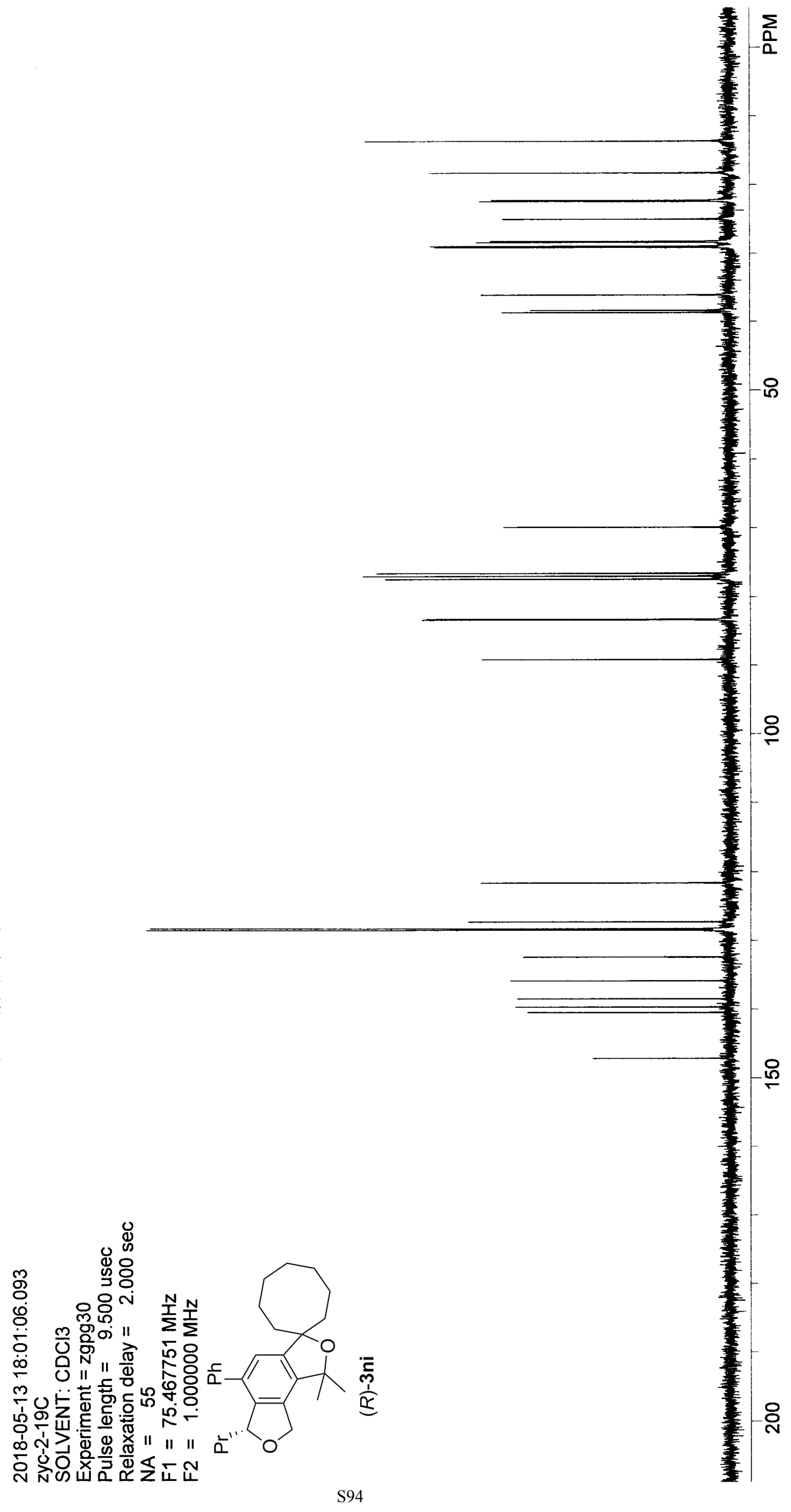


$$
\text { zyc-2-13-ia-400-1-1-214 }
$$

Acquired Time: 2018-05-23, 15:29:57

Date File: D: \zhuguangjiong $\backslash z y z \backslash 20180522 \backslash z y c-2-13-$

Printed Time: 2018-05-23, 17:09:24

ia-400-1-1-214. org

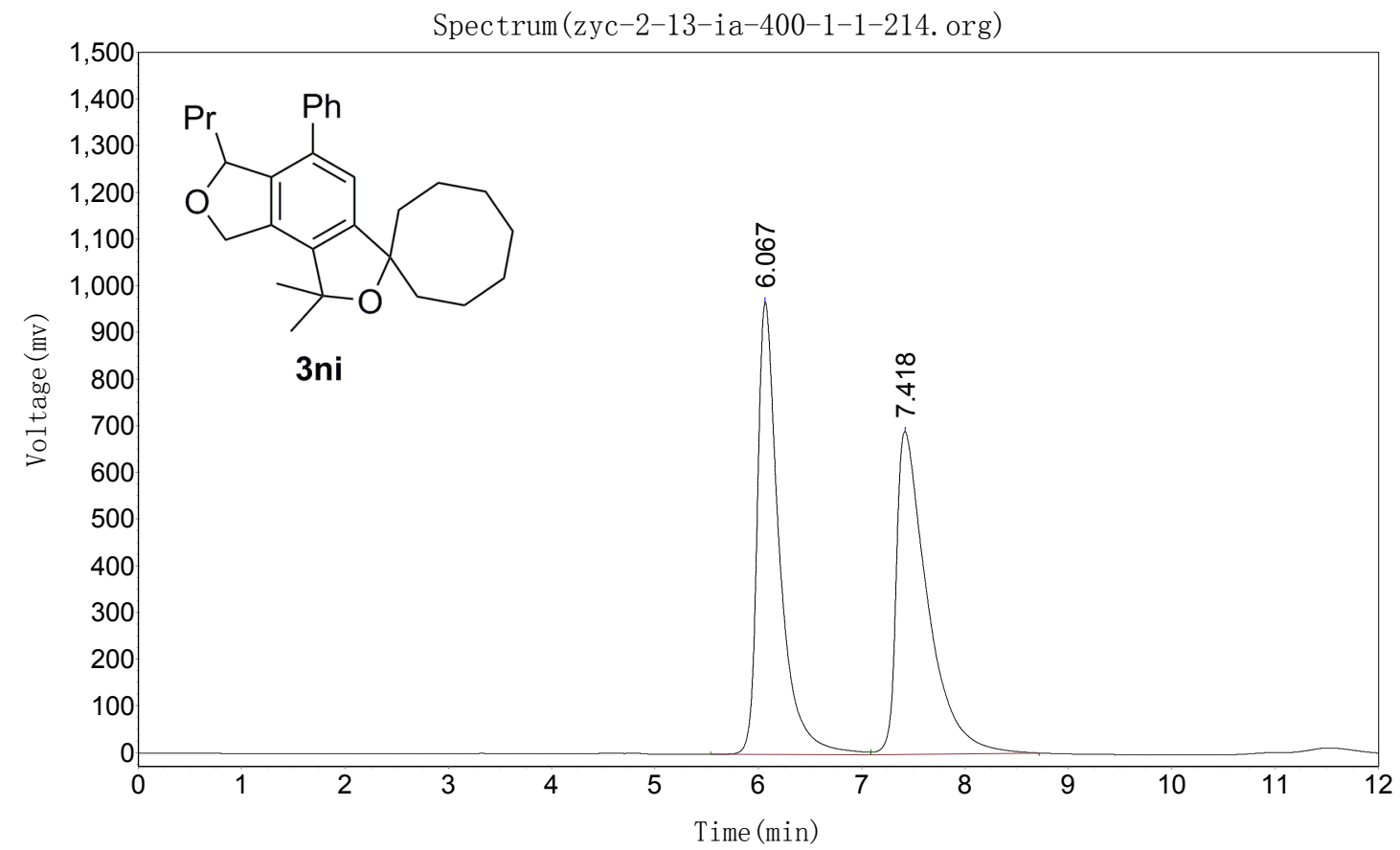

Analysis Results

\begin{tabular}{|c|c|c|c|c|c|}
\hline NO. & Name & $\mathrm{RT}$ [min] & Height & Area & Area\% \\
\hline 1 & & 6.067 & 969538.125 & 14366822.000 & 49.7632 \\
\hline 2 & & 7. 418 & 691516.688 & 14503534.000 & 50.2368 \\
\hline Total & & & 1661054.813 & 28870356.000 & 100.0000 \\
\hline
\end{tabular}




$$
\text { zyc-2-19-ia-400-1-1-214 }
$$

Acquired Time: 2018-05-23, 16:08:01

Date File: D: \zhuguangjiong $\backslash z y z \backslash 20180522 \backslash z y c-2-19-$

Printed Time: 2018-05-23, 18:10:08

ia-400-1-1-214. org

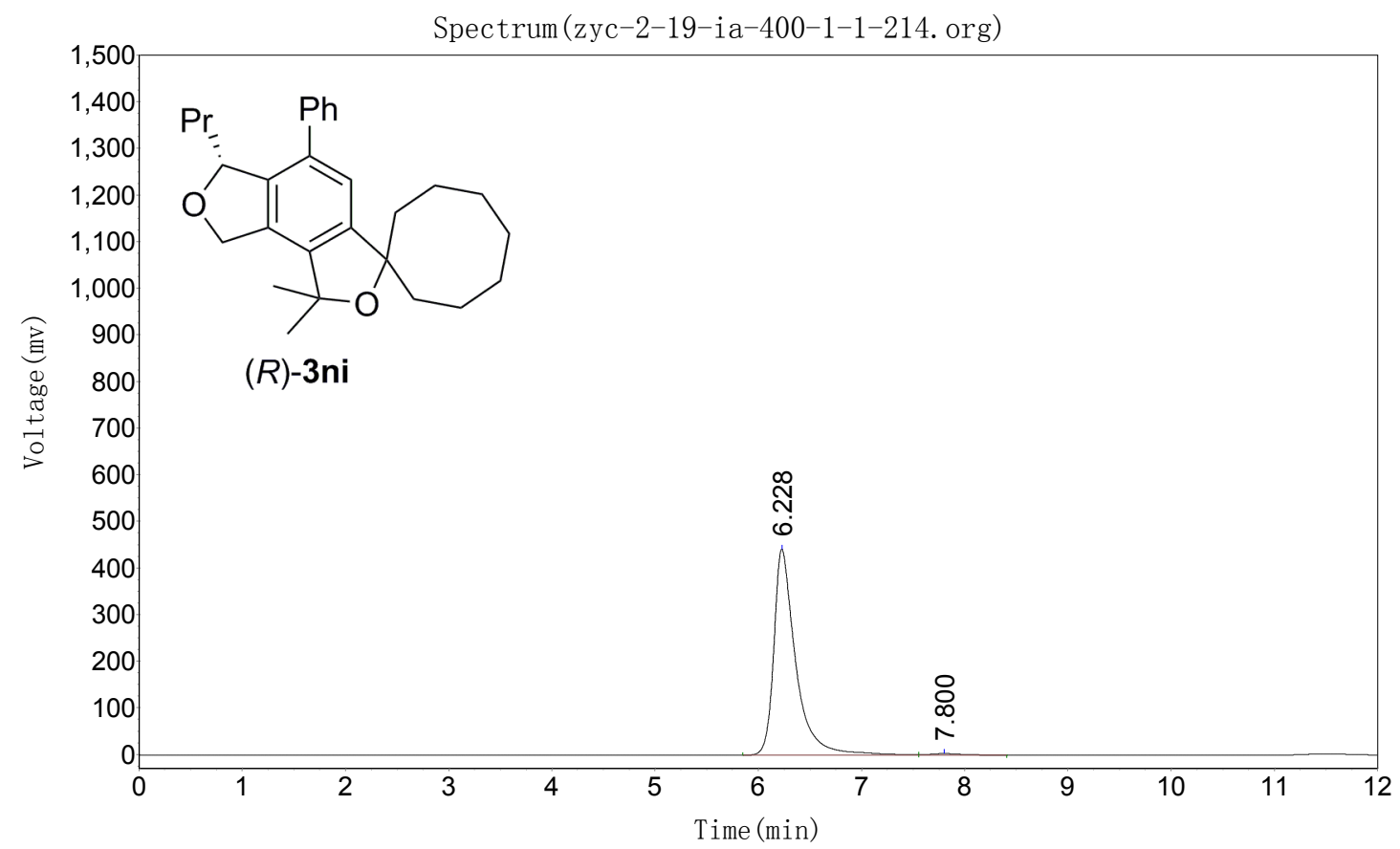

Analysis Results

\begin{tabular}{|c|c|c|c|c|c|}
\hline No. & Name & $\mathrm{RT}$ [min] & Height & Area & Area\% \\
\hline 1 & & 6.228 & 442368.281 & 6554973.000 & 98.8059 \\
\hline 2 & & 7.800 & 3451.176 & 79217. 922 & 1. 1941 \\
\hline Total & & & 445819.457 & 6634190.922 & 100.0000 \\
\hline
\end{tabular}



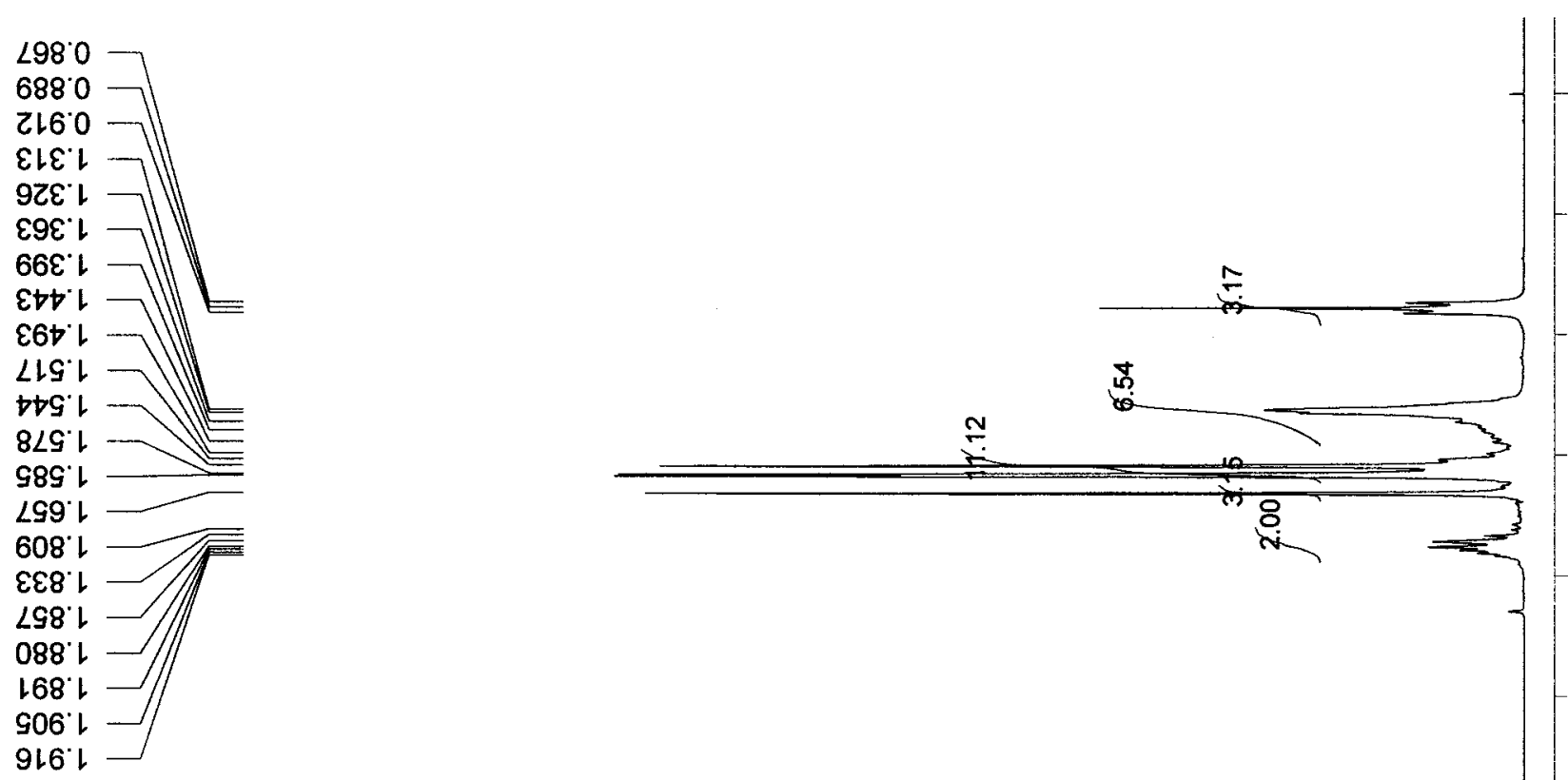

9 . 6
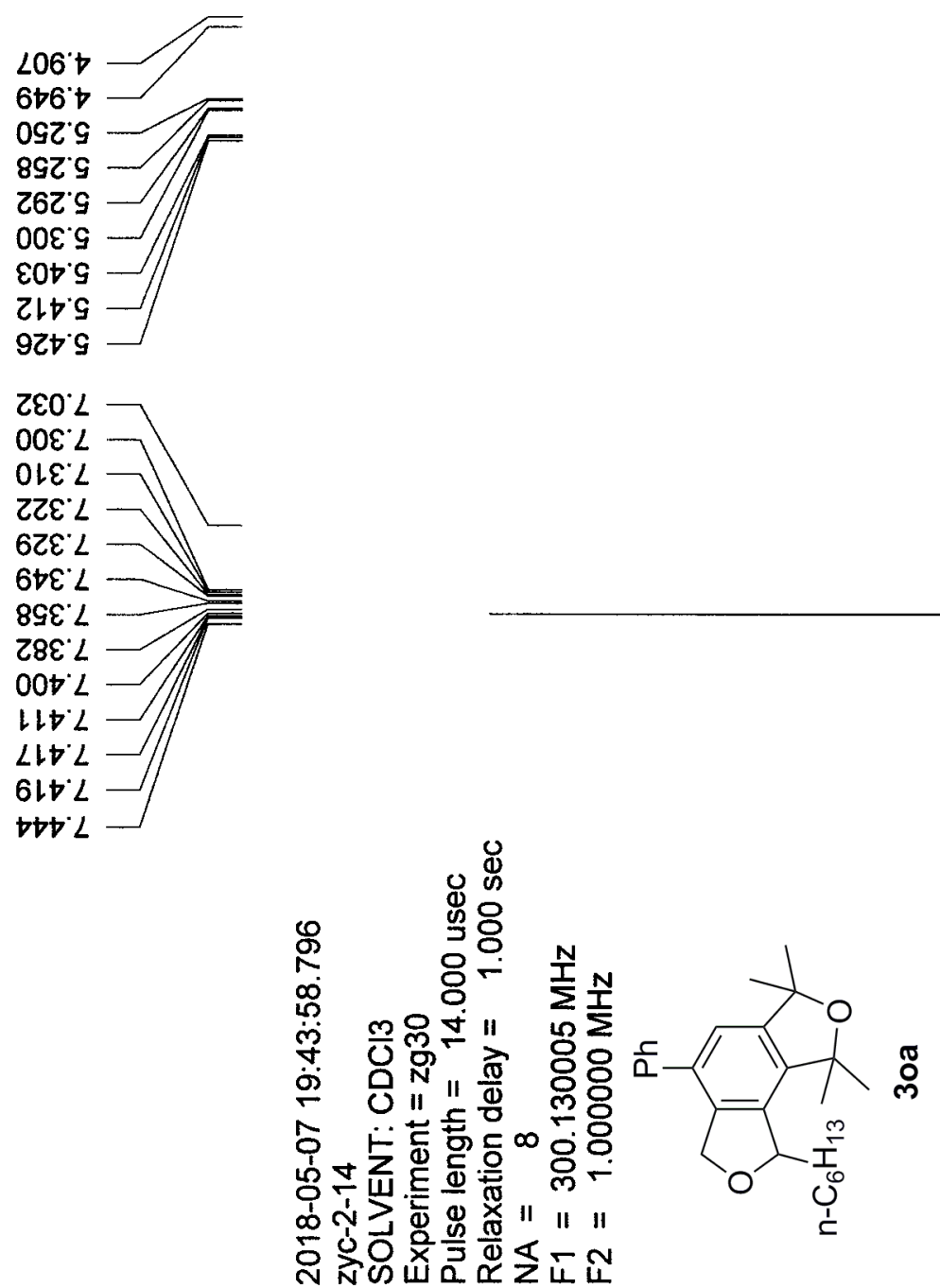

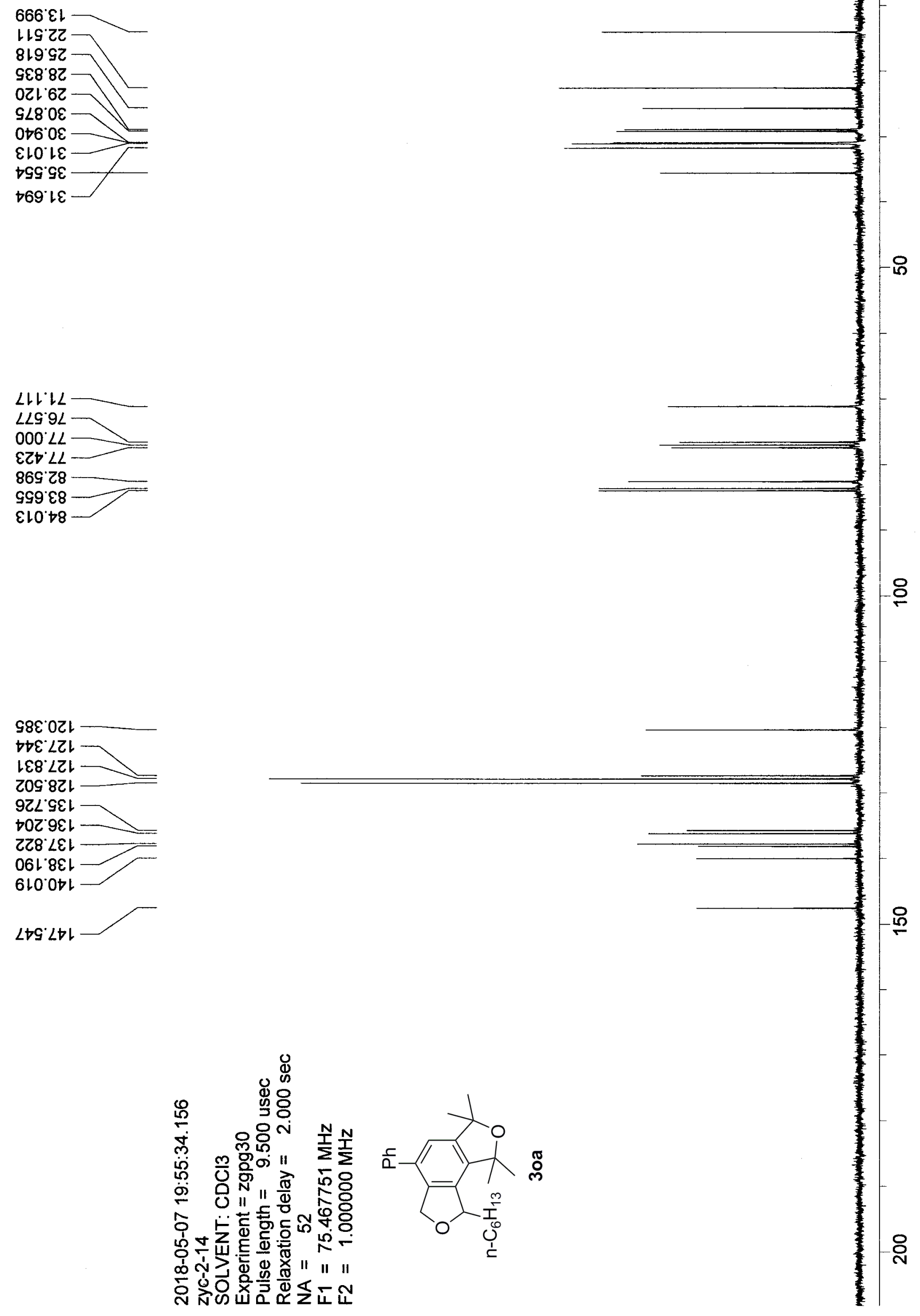

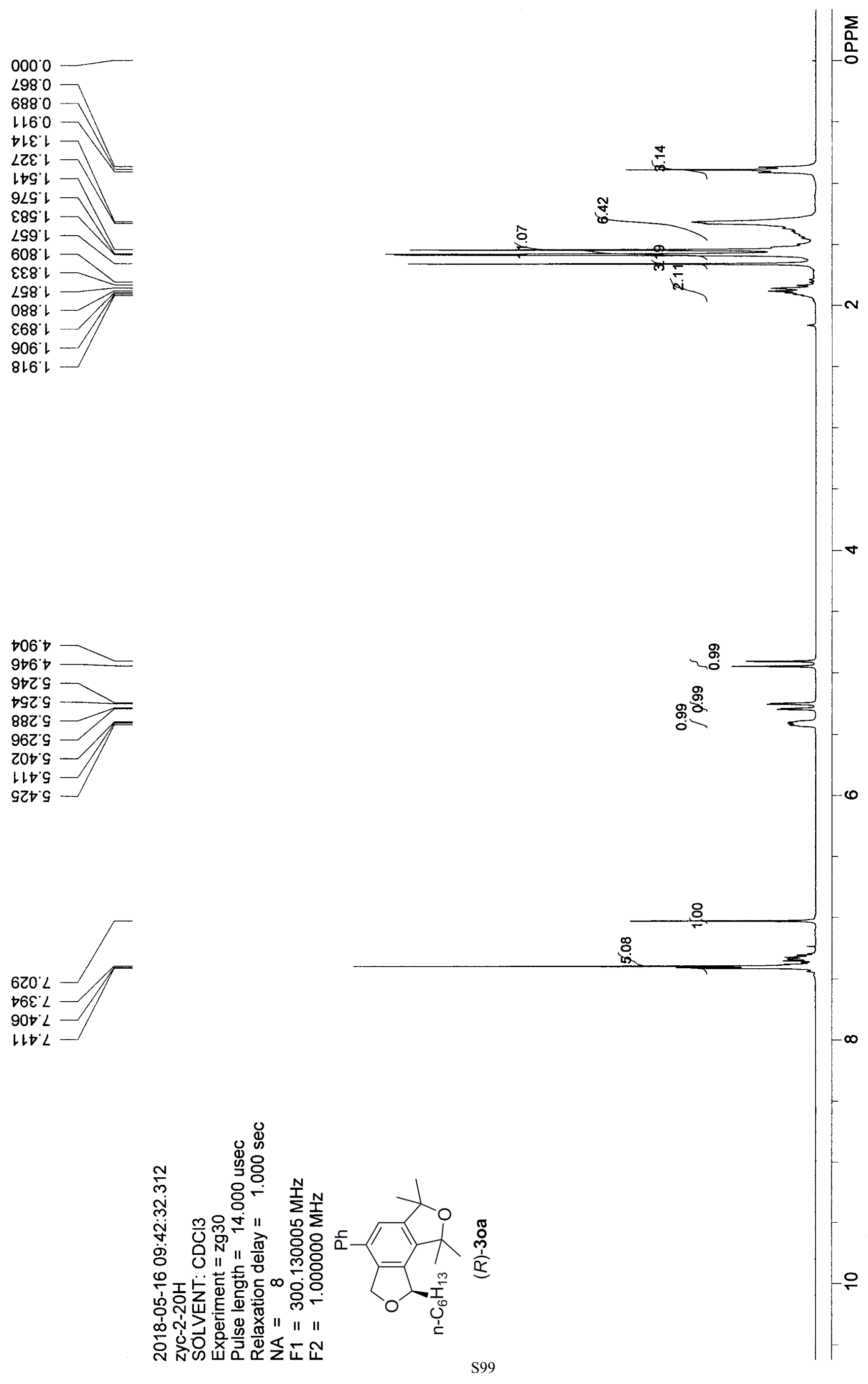

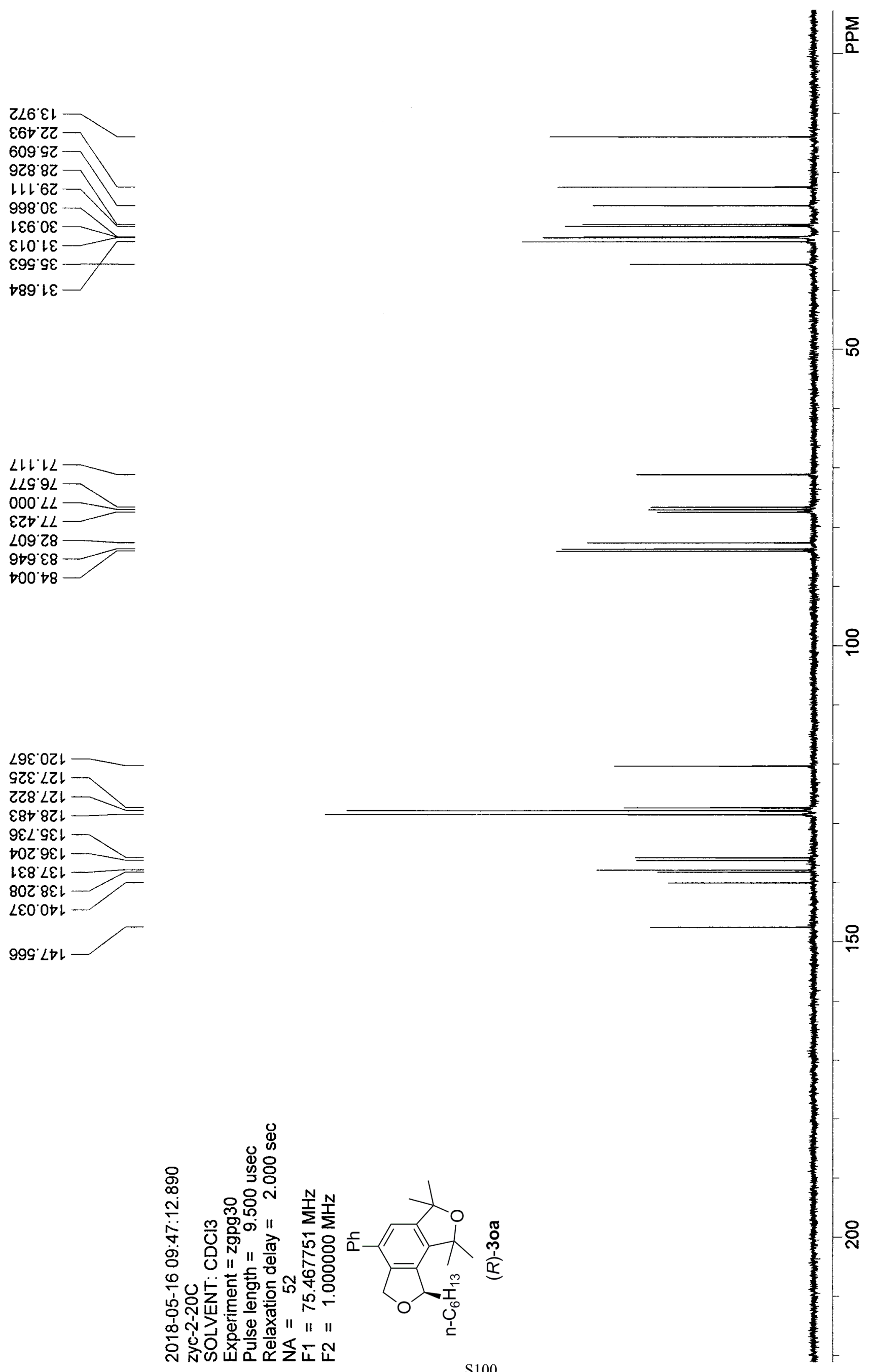


$$
\text { zyc-2-14-ia-400-1-1-214 }
$$

Acquired Time: 2018-05-23, 16:47:04

Date File: D: \zhuguangjiong $\backslash z y z \backslash 20180522 \backslash z y c-2-14-$

Printed Time: 2018-05-23, 17:10:27

ia-400-1-1-214. org

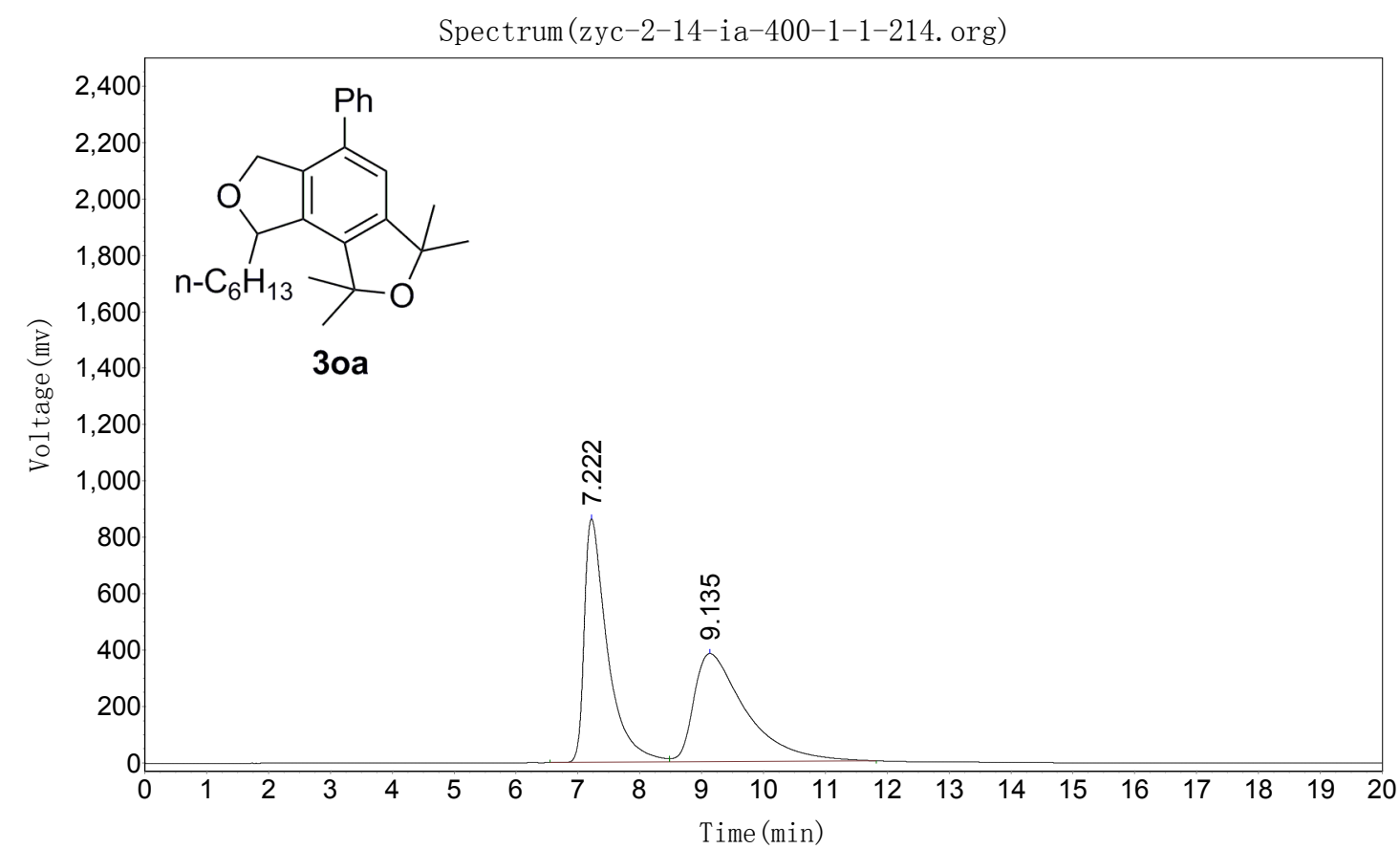

Analysis Results

\begin{tabular}{|c|c|c|c|c|c|}
\hline NO. & Name & $\mathrm{RT}$ [min] & Height & Area & Area\% \\
\hline$\overline{1}$ & & 7.222 & 862804.938 & 22447920.000 & 50.2705 \\
\hline 2 & & 9. 135 & 383426.844 & 22206312.000 & 49. 7295 \\
\hline Total & & & 1246231.781 & 44654232.000 & 100.0000 \\
\hline
\end{tabular}




$$
\text { zyc-2-20-ia-400-1-1-214 }
$$

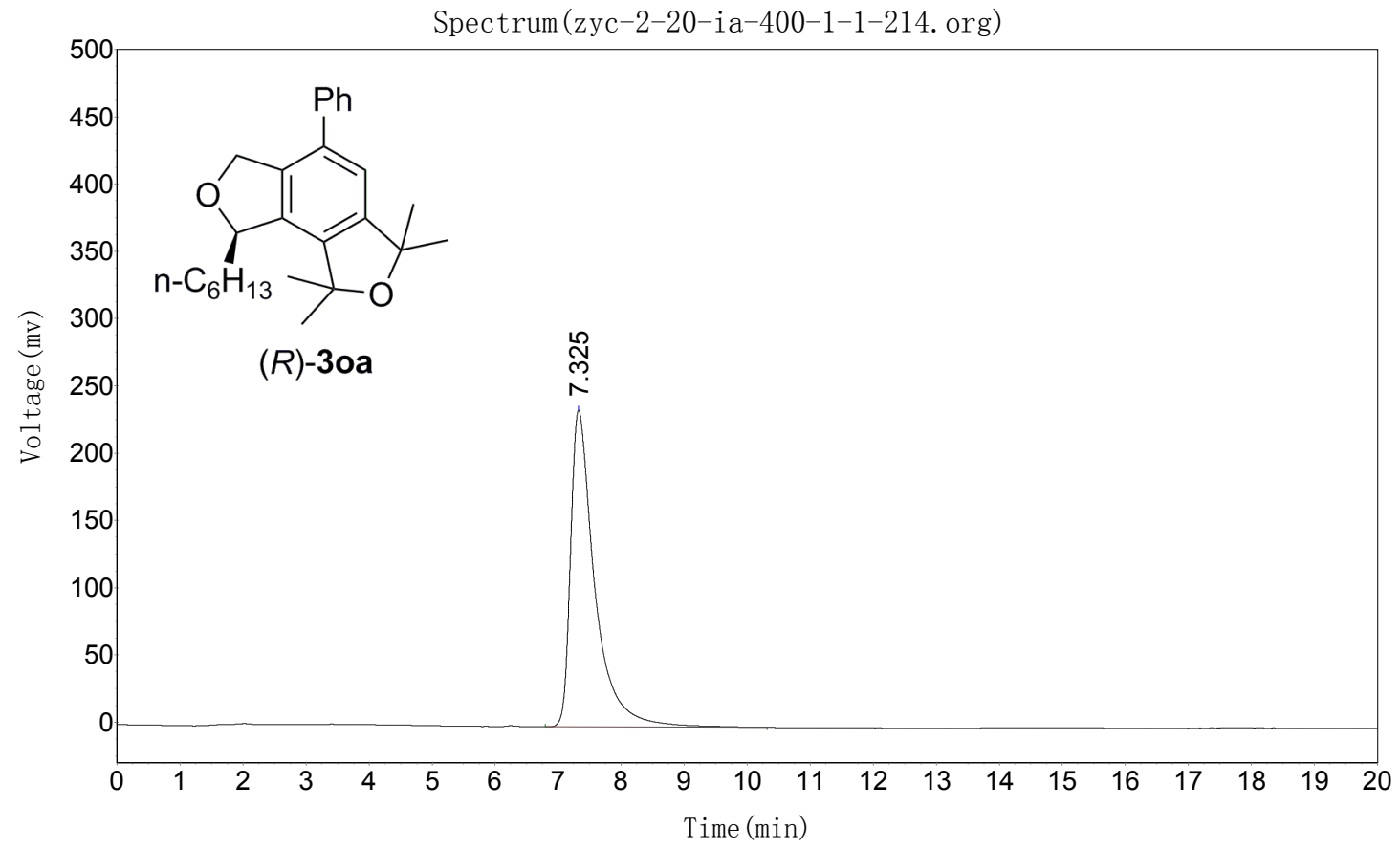

Analysis Results

\begin{tabular}{|c|c|c|c|c|c|}
\hline NO. & Name & $\mathrm{RT}$ [min] & Height & Area & Area\% \\
\hline 1 & & 7.325 & 235586.078 & 6304646.000 & 100.0000 \\
\hline Total & & & 235586.078 & 6304646.000 & 100.0000 \\
\hline
\end{tabular}




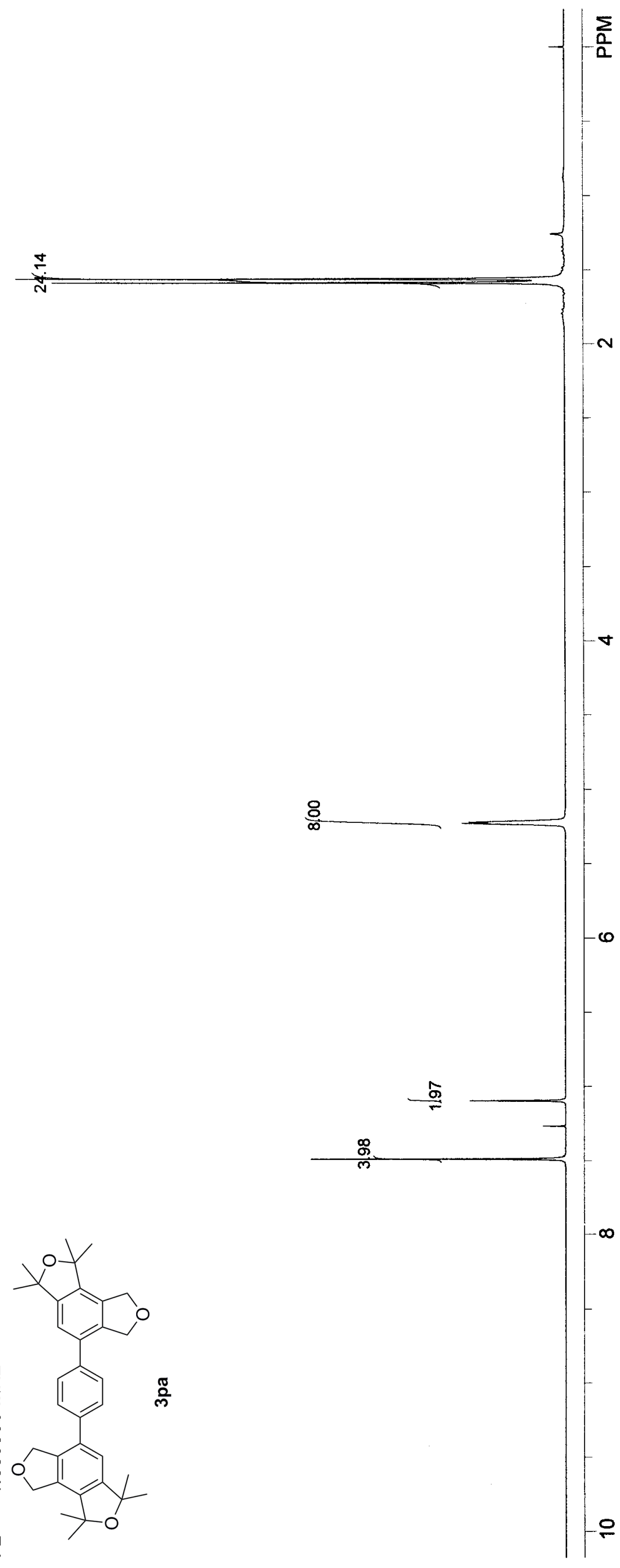

699 ᄂ

S89'

$022^{\circ} 9$

$8 Z Z^{\circ} 9$

S60 L

$68 t 2$

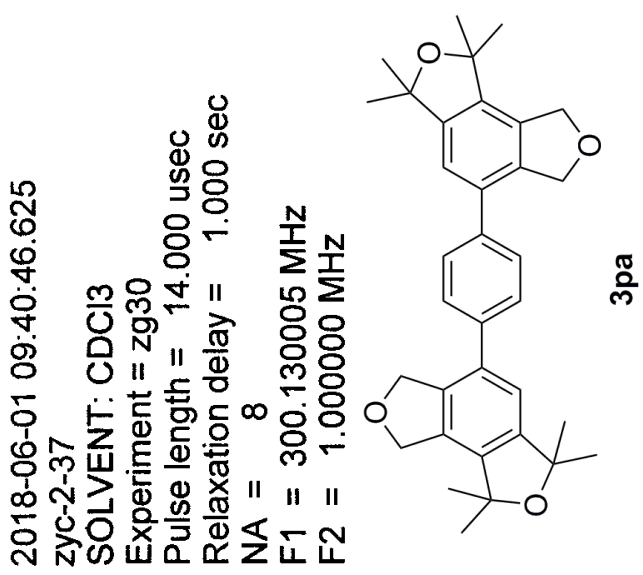




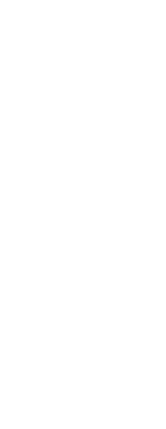

89E.0ZL

ZSเ8ZL

8Z9'Zह

Z60' $9 \& 1$

$\forall \forall \varepsilon^{\circ} \angle \varepsilon L$

$\neg 69^{\circ} 8 \varepsilon \downarrow$

0ะ๕ $6 \varepsilon \downarrow$

$86 l \angle b l$

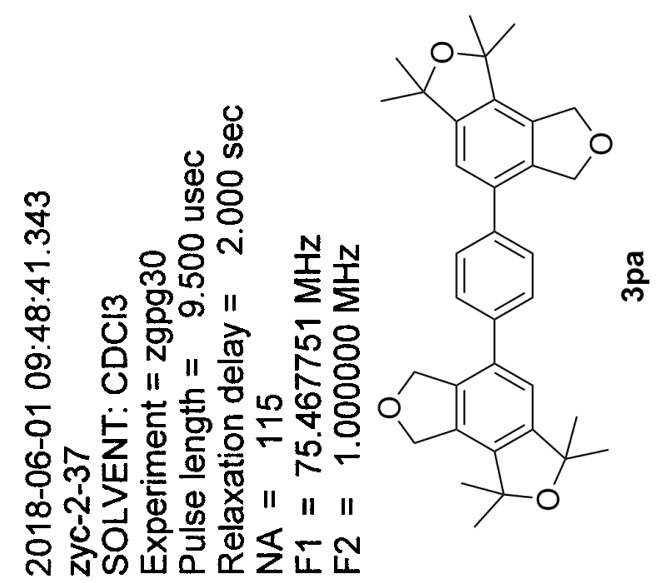

